\title{
DEVELOPMENT AND CHARACTERIZATION OF AN IN- HOUSE CUSTOM BIOREACTOR FOR THE CULTIVATION OF A TISSUE ENGINEERED BLOOD-BRAIN BARRIER
}

\author{
A Thesis \\ Presented to the Faculty of California Polytechnic State University \\ San Luis Obispo
}

In Partial Fulfillment of a Requirements for the Degree Master of Science in Biomedical Engineering

By Amin Mirzaaghaeian

July 2012 
(C) 2012

Amin Hadi Mirzaaghaeian

\section{ALL RIGHTS RESERVED}




\section{COMMITTEE MEMBERSHIP}

TITLE: $\quad$ Development and Characterization of an In-House Custom Bioreactor for the Cultivation of a Tissue Engineered

Blood-Brain Barrier

AUTHOR: Amin Hadi Mirzaaghaeian

DATE SUBMITTED: July 2012

COMMITEE CHAIR: Dr. Kristen O’Halloran Cardinal

COMMITTEE MEMBER: Dr. Trevor Cardinal

COMMITTEE MEMBER: Dr. Lily Laiho 
ABSTRACT

\title{
DEVELOPMENT AND CHARACTERIZATION OF AN IN- HOUSE CUSTOM BIOREACTOR FOR THE CULTIVATION OF A TISSUE ENGINEERED BLOOD-BRAIN BARRIER
}

\begin{abstract}
Amin Mirzaaghaeian
The development of treatments for neurological disorders such as Alzheimer's and Parkinson's disease begins by understanding what these diseases affect and the consequences of further manifestation. One particular region where these diseases can produce substantial problems is the blood-brain barrier (BBB). The BBB is the selective diffusion barrier between the circulating blood and the brain. The barrier's main function is to maintain CNS homeostasis and protect the brain from the extracellular environment. The progression of $\mathrm{BBB}$ research has advanced to the point where many have modeled the BBB in vitro with aims of further characterizing and testing the barrier. Particularly, the pharmaceutical industry has gained interest in this field of research to improve drug development and obtain novel treatments for patients so the need for an improved model of the BBB is pertinent in their discovery. In the Cal Poly Tissue Engineering lab, an in vitro tissue engineered BBB system has previously been obtained and characterized for the initial investigation of the barrier and its components. However, certain limitations existed with use of the commercial system. Therefore, the focus of this thesis was to improve upon the capabilities and limitations of this commercialized system to allow further expansion of BBB research. The work performed was based on three aims: first to design and develop an in-house bioreactor system that could be used to cultivate the BBB; second, to characterize flow and functional capabilities of the bioreactor; third, to
\end{abstract}


develop protocols for the overall use of the bioreactor, to ultimately allow co-cultures of BAEC and C6 glioma cells, and further the progression toward creating an in vitro model of the BBB.

The work of this thesis demonstrates development of an in-house custom bioreactor system that can successfully culture cells. Results showed that the system was reusable, could be sterilized and monitored, was easily used by students trained in the laboratory, and allowed non-destructive scaffold extraction. This thesis also discusses the next set of experiments that will lead to an in vitro model of the BBB. 


\section{ACKNOWLEDGEMENTS}

First, I would like to acknowledge and thank my thesis advisor Dr. Kristen O'Halloran Cardinal. Thank you for giving me the opportunity to work with you and the Tissue Engineering Lab. It was a great privilege working with you, and wish you the best in future research. In addition, I would like to thank: Dr. Trevor Cardinal and Dr. Lily Laiho for being members of my thesis defense committee and being excellent teachers.

I would like to acknowledge the faculty and staff of the Biomedical Engineering Department at Cal Poly, including Dr. David Clague and David Laiho.

Dr. Clague- Thank you for believing in me and always having the time to talk. I will always remember the humor you brought to lecture, and wish you the best. Mr. LaihoThank you for your guidance in manufacturing and design, it will provide me with the skills that I will need to continue to practice engineering.

I would also like to acknowledge the help and support of my fellow 2012 Spring Tissue Engineering Lab group members: Marcus Foley, Mike Gibbons, Rachel Gohres, Mike Machado, Deven Patel, and Sarah Ur. Marcus- You were a great lab manager, thank you for all the help. I am excited to start Genicell with you and hope for the best! Mike GThank you for your patience when first using and evaluating the initial bioreactor! Rachel- Thank you for being the extra pair of hands for some experiments, you helped me avoid many spills! Mike M- Thank you for always reminding me that esthetics of a design can change everything. Let's make the dream of starting a company into a reality! Deven- Thank you for making all of the scaffolds ever used in the duration of this project! Sarah- Thank you for being an awesome BVM bioreactor reference!

To my family- Dad, Mom, Ameh Nahid, Grandpa, and Grandma. Dad- Thank you for being my inspiration, my strength, my life. Mom-Thank you for your strength and know that your son could never have done this without you. Ameh Nahid- Thank you for your guidance and wisdom. Grandparents- Thank you for your love and always telling to reach for the stars. 


\section{Table of Contents}

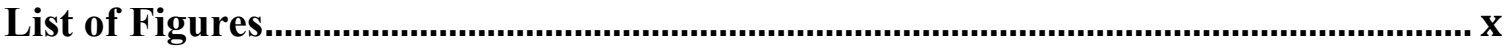

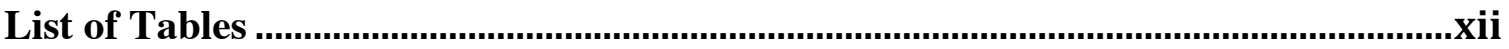

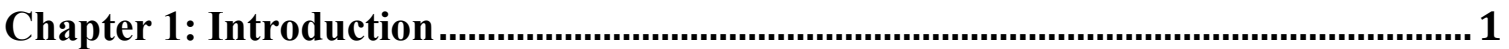

1.1 The Blood-Brain Barrier................................................................................... 1

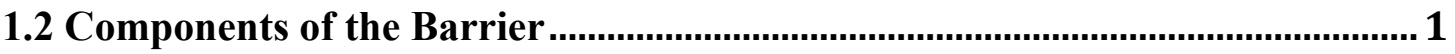

1.2.1 Brain Microvascular Endothelial Cells........................................................... 2

1.2.2 Basement Membrane............................................................................................... 3

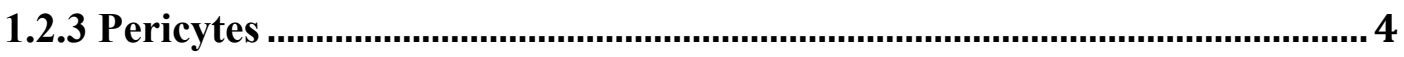

1.2.4 Glial Cells...................................................................................................... 4

1.3 The Importance of Modeling the BBB ............................................................ 5

1.4 Approaches to Modeling the Blood-Brain Barrier............................................. 6

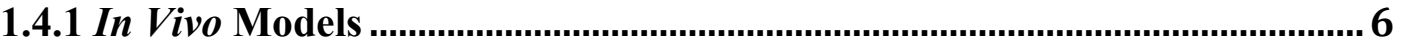

1.4.2 In Situ Model.................................................................................................... 7

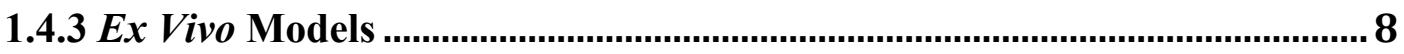

1.4.4 In silico Models ............................................................................................ 9

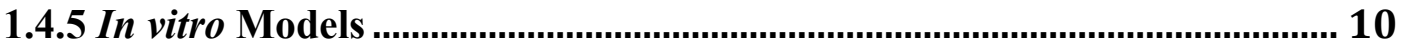

1.5 Bridging the Gap Between In vivo and In vitro Models................................... 15

1.6 Academia's Contribution..................................................................................... 16

1.7 Summary of Overall Thesis Goals ...................................................................... 18

Chapter 2: Initial Design of BBB Bioreactor, Manufacturing, \& Costs ................... 20

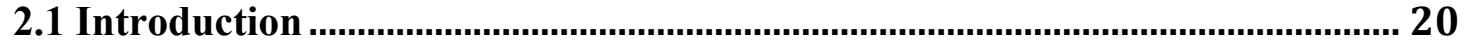

2.2 Initial Designs of the BBB Bioreactor ........................................................... 21

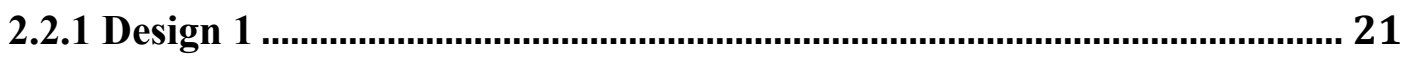

2.2.2 Evaluation of Design 1 ........................................................................ 23

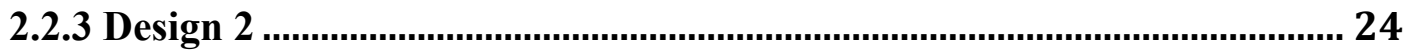

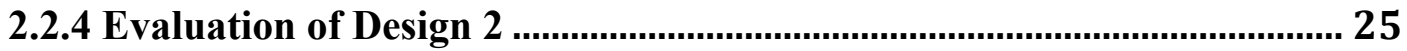

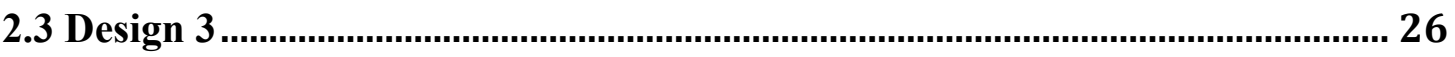

2.4 Manufacturing........................................................................................................ 27

2.5 Machined Parts and Bioreactor Components …................................................ 30

2.5.1 Outer Caps............................................................................................. 30

2.5.2 Scaffold Caps \& Stabilization Bar .............................................................. 31 
2.5.3 Scaffolds \& Barbs ............................................................................................. 32

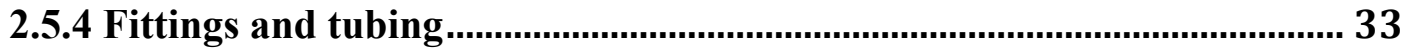

2.5.5 Media Reservoir \& Perfusion Pump....................................................... 34

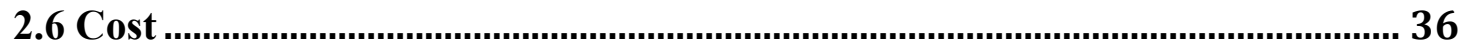

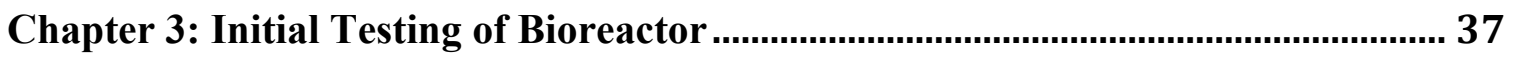

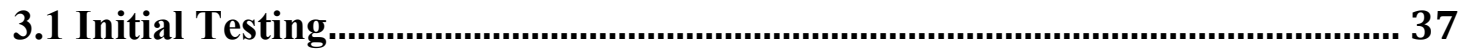

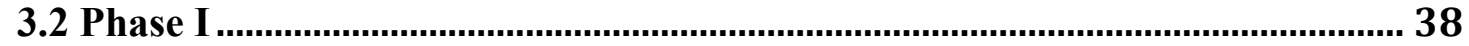

3.2.1 Leak Tests ................................................................................................ 38

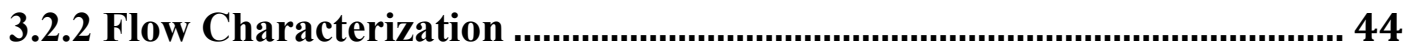

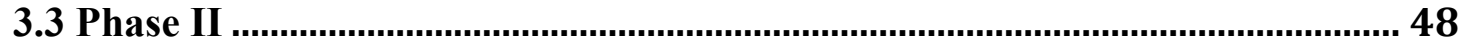

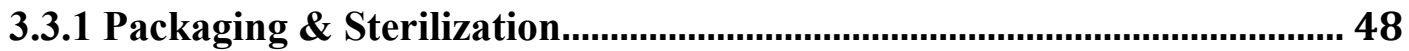

3.4 Phase III.................................................................................................................. 52

Chapter 4: Final Design of BBB Bioreactor .............................................................. 55

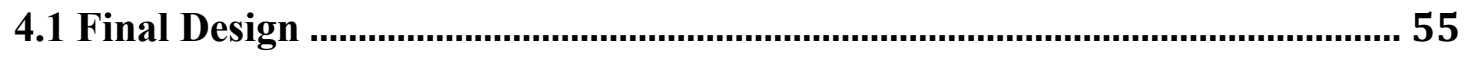

4.2 Components .......................................................................................................... 55

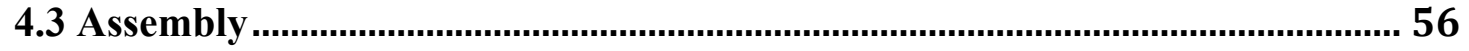

4.3.1 Scaffold Cap Preparation ........................................................................... 57

4.3.2 Outer Cap Installation .................................................................... 58

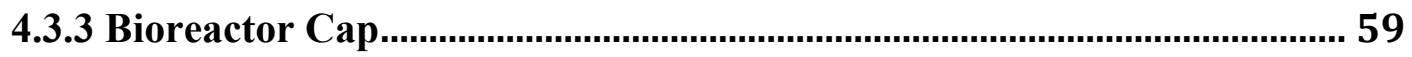

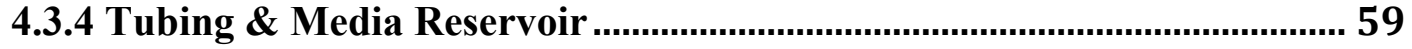

Chapter 5: Cell Testing Methods, Results, \& Discussion ................................................ 61

5.1 Introduction ............................................................................................................ 61

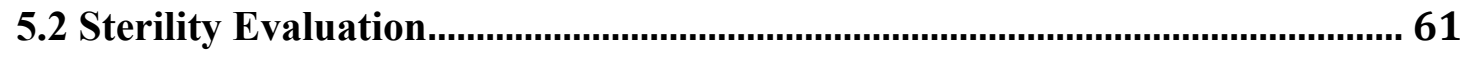

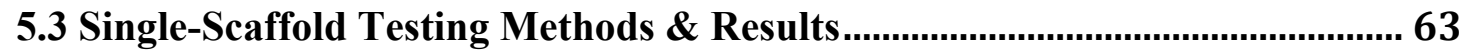

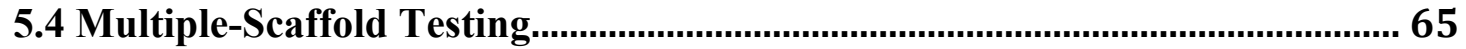

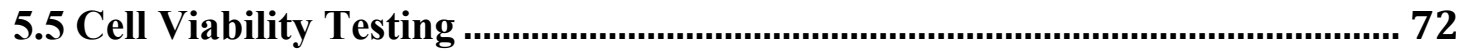

Chapter 6: Final Discussion, Future Work, Limitations, \& Conclusion.................... 75

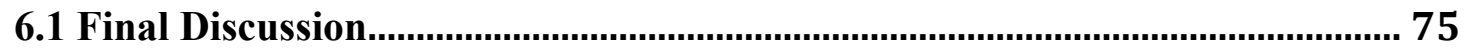

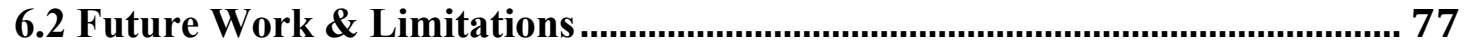

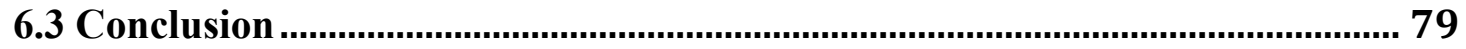

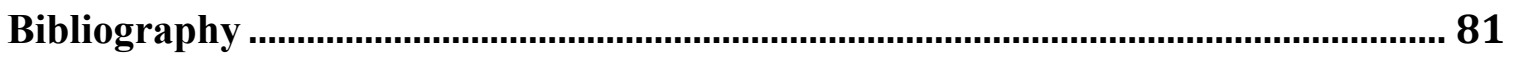

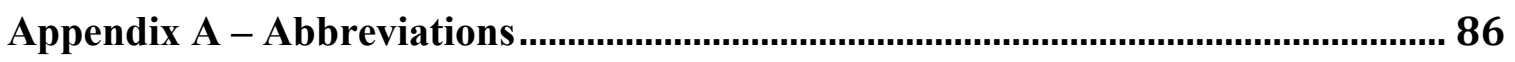


Appendix B - Parts Information and Design Drawings .......................................... 87

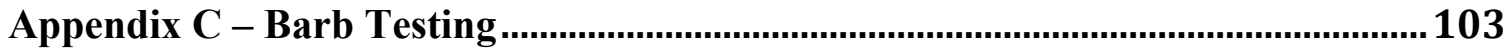

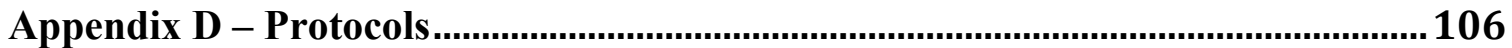

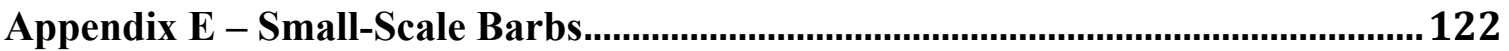




\section{List of Figures}

Figure 1: Diagram of the associated components of the BBB..................................... 1

Figure 2: Animation of the neurovascular unit....................................................... 2

Figure 3: Schematic of work performed by Hatherell et al ..................................... 12

Figure 4: Schematic of one dynamic culture method ................................................... 13

Figure 5: Cellco Inc. hollow fiber bioreactor ........................................................................ 13

Figure 6: Custom bioreactor developed by Cucullo et al............................................. 14

Figure 7: CellMax Duo Bioreactor........................................................................ 17

Figure 8: SolidWorks drawing of the first design of the bioreactor ............................ 22

Figure 9: Solid drawing of cartridge ...................................................................... 24

Figure 10: Solid Drawing created prior to manufacturing ............................................. 27

Figure 11: Outer housing machined in-house ........................................................ 29

Figure 12: Schematic of internal regions of the outer cap. ........................................ 30

Figure 13: Machined outer caps...................................................................................... 31

Figure 14: Machined scaffold caps with and without stabilization bar ..................... 32

Figure 15: Qosina barb..................................................................................... 33

Figure 16: PLGA scaffold. .................................................................................................... 33

Figure 17: Diagram of all fittings used in bioreactor system .................................... 34

Figure 18: Media reservoir .......................................................................................................... 35

Figure 19: Pump system .................................................................................................. 35

Figure 20: Initial bioreactor .......................................................................................... 37

Figure 21: O-ring picks. ............................................................................................................... 38

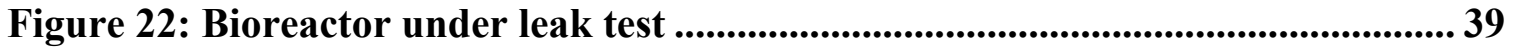

Figure 23: Second-generation housing......................................................................... 40

Figure 24: Second-generation bioreactor housing with cartridge............................... 42

Figure 26: Assembled bioreactor..................................................................................... 42

Figure 27: Final solution to sealing the bioreactor ......................................................... 43

Figure 28: Custom internal O-ring. ....................................................................... 44

Figure 29: Three Teflon bars........................................................................................ 45

Figure 30: Flow rates in $\mathrm{ml} / \mathrm{min}$......................................................................................... 45

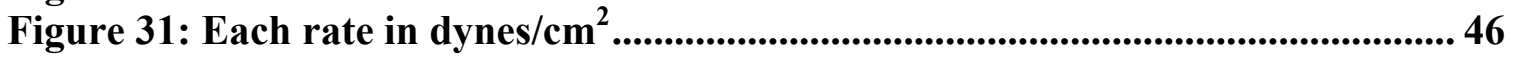

Figure 32: Bioreactor packed .......................................................................................... 49

Figure 33: Bioreactor system packaged ........................................................................... 49

Figure 34: Bioreactor packages for sterilization .......................................................5 50

Figure 35: Funnel technique .................................................................................... 52

Figure 36: Fully submerge scaffold barbs. ......................................................................... 53

Figure 37: Final design of bioreactor system ..................................................................... 55

Figure 39: Scaffold caps installed ...................................................................................5 57

Figure 40: Scaffold sutured to two Qosina barbs ............................................................ 58

Figure 41: Scaffold installation into bioreactor. ................................................................ 58

Figure 42: Inlet Tubing ...................................................................................................6 60

Figure 43: Primed bioreactor system............................................................................... 62

Figure 44: Contaminated bioreactor.....................................................................................64 64

Figure 45: BBI Images of single-scaffold short-term test........................................... 65

Figure 46: BBI Images of single-scaffold long-term test. ...........................................65

Figure 47: Scaffold configuration of four scaffolds. ...................................................... 66 
Figure 48: Box created to count cells. ........................................................................6 67

Figure 49: BBI Image of bottom scaffold. ...........................................................6 68

Figure 50: Graph of total cell number. ............................................................... 70

Figure 51: Graph of average cell densities. ................................................................ 71

Figure 52: Cell viaibility images. ................................................................................ 73 


\section{List of Tables}

Table I: Design evaluation for initial design 1............................................................ 24

Table II: Design Evaluation for initial design 2 .................................................... 26

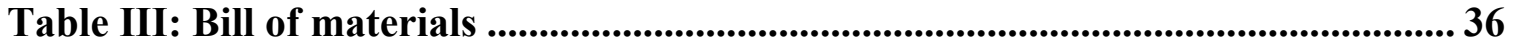

Table IV: List of O-rings tested ................................................................................ 39

Table V: Table displaying flow rates in $\mathrm{ml} / \mathrm{min}$ and shear stress in $\mathrm{dynes} / \mathrm{cm}^{2}$........ 47

Table VI: Flow rate characterization performed by Bryan Brandon ........................ 47

Table VII: Evaluation of Sterile and Non-sterile protocols ....................................... 63

Table VIII: Tukey test evaluating which proximal region.......................................6 69

Table IX: Tukey test evaluating which middle region .............................................69 69

Table X: Table showing results obtained from cell counter.. ....................................... 73 


\section{Chapter 1: Introduction}

\subsection{The Blood-Brain Barrier}

Over a century has passed since the term "blood-brain barrier" was first used [1], and today numerous studies can confidently describe the major functions of the blood-brain barrier (BBB). The barrier's main function is to maintain CNS homeostasis by providing a perpetual supply of nutrients through specified transport mechanisms, regulating inflammatory cells in response to changes in the local environment, and protecting the brain from the extracellular environment $[2,3,4]$. Characterization of these functions became possible from understanding the components of the BBB including endothelial cells, pericytes, basement membrane, and neural cells (Fig. 1).

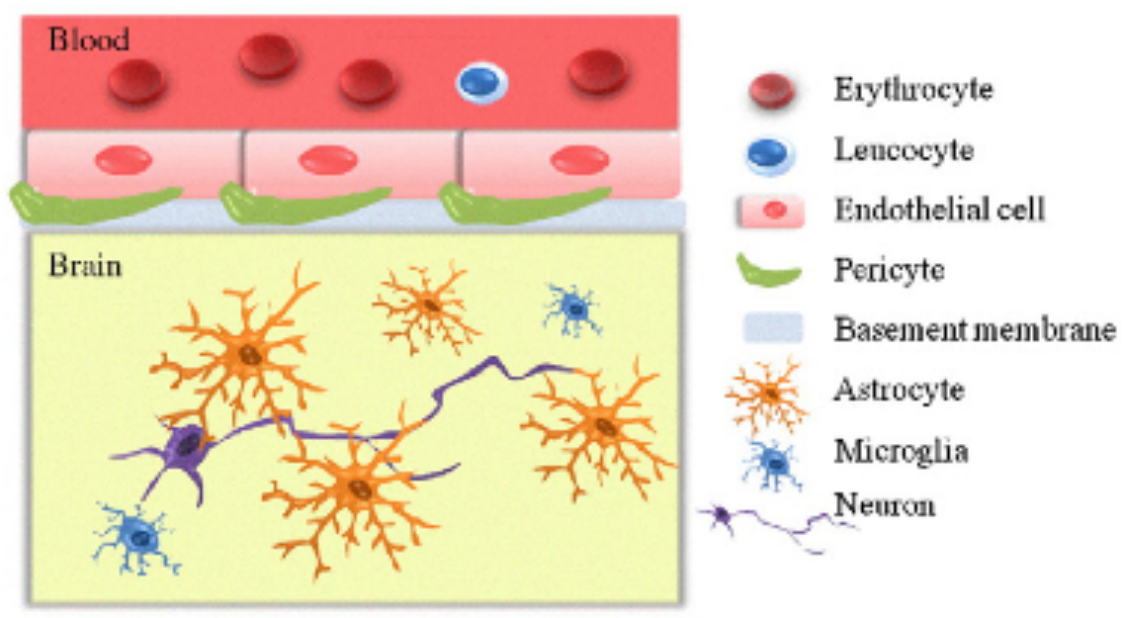

Figure 1: Diagram of the associated components of the BBB, illustrating the location and orientation of each component relative to blood flow [4].

\subsection{Components of the Barrier}

The permeability of the barrier is dependent on the presence of the brain

microvascular endothelial cells (BMVEC) [4]. However, the integrity of the barrier relies on the complementary components of the neurovascular unit (NVU) [5]. The NVU 
consists of BMVECs, a basement membrane, pericytes, astrocytes, and microglia; each of these will be explained in the following section (Fig. 2) [1]. Each component plays a contributing role in either the integrity of the barrier's permeability or BMVEC phenotype expression.

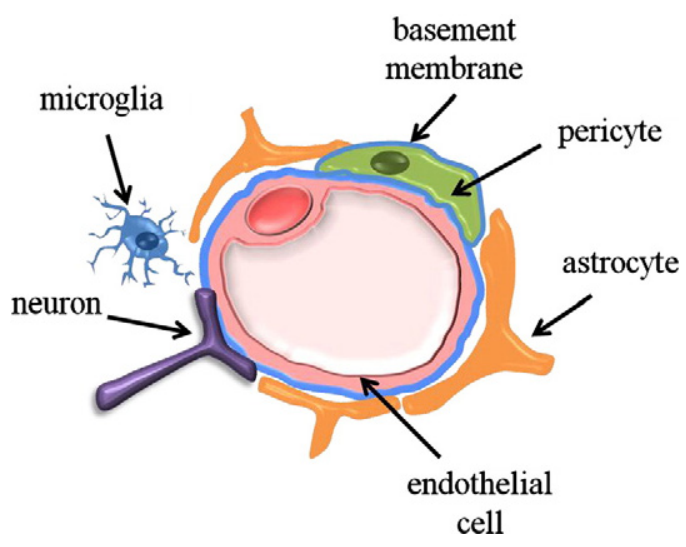

Figure 2: Animation of the neurovascular unit comprised of endothelial cells, a basement membrane, pericytes, astrocyte, microglia, and neurons [1].

\subsubsection{Brain Microvascular Endothelial Cells}

BMVECs represent the anatomic basis and the primary component of the BBB

$[6,7]$, and are the only component of the NVU that contacts the circulating blood. The endothelial cells (ECs) mediate metabolism and selective transport of substances from blood to the brain or vice versa from the parenchyma into the circulation. The EC's ability to communicate with the surrounding components results in maintenance and amplification of proper brain homeostasis [8]. BMVECs differ from other vascular ECs in that they display a higher expression of certain characteristics by being a part of the BBB. Specifically, BMVECs are fifty to one hundred times tighter compared to the rest of the peripheral system, due to the formation of tight junctions (TJs) between adjacent ECs, causing inhibition of certain hydrophilic solutes [9]. The BMVEC lining is 
comprised of junctional complexes including TJs and adherens junction proteins (AJs)

that remove gaps between cells preventing free diffusion of substances from the circulation $[10,11]$. The overall structure of BMVECs differs from ECs of the peripheral capillaries, as they are absent of fenestrae, perform minimal pinocytosis, and are surrounded by a uniform basement membrane $[12,13]$. The brain endothelium also contains a higher number of mitochondria, allowing increased energy potential to perform selective transport into and out of the brain $[4,13]$. Transmembrane proteins from the cellular adhesion molecule family including selectins, immunoglobulins, and integrins, participate in intercellular adhesion and focal adhesion to the basement membrane $[14,15,16]$.

\subsubsection{Basement Membrane}

The basement membrane maintains a connection between the surrounding components and BMVECs by stabilizing the ECs and surrounding pericytes [17]. The membrane is composed of structural proteins including collagen and elastin, specialized proteins such as fibronectin and laminin, and proteoglycans $[18,15]$. Cellular adhesion molecules seen in BMVECs have additional signaling proteins and are pertinent to the maintenance of the BBB [17]. A study performed by Carvey et al. investigated several dysfunctions including damage to the basement membrane and its affect on BMVEC cytoskeleton. The researchers showed that damage to the basement membrane lead to a decrease in tight junction efficiency and barrier performance [17]. 


\subsubsection{Pericytes}

Sharing the basement membrane, but located on endothelial cells, lay pericytes that are not just specific to BMVECs, but can be found in other capillaries and postcapillary venules. Pericytes are also known as vascular smooth muscle cells or myofibroblasts, and cover $22-32 \%$ of capillaries [19]. However, pericytes in the cerebral region express a higher degree of coverage based on the tightness of the intercellular junctions [20]. Communication between pericytes and ECs exists due to the presence of gap junctions, TJs, adhesion plaques, and soluble factors [21, 22]. Claims have been made indicating that pericytes have influential factors on ECs through their ability to communicate. Persidsky et al. illustrated the ability for pericytes to modulate BMVEC migration, proliferation, and differentiation, all of which can increase the integrity of the BBB [4]. Pericytes can also affect the permeability of the BBB due to environmental changes. During hypoxia or traumatic brain injuries, pericytes have been seen to migrate away from the BMVECs, increasing permeability of the barrier [23].

\subsubsection{Glial Cells}

Glial cells are the remaining components of the NVU that contribute to the function and properties of the BBB [1]. Glial cells include astrocytes and microglia cells. Astrocytes have been seen to restore the barrier's function and properties once added in co-culture with BMVECs [24]. Through this observation, astrocytes can be seen to modulate neurovascular structure and integrity [25]. Microglia contribute to the immune response of the CNS, altering their phenotype in response to changes in homeostasis. Choi et al. hypothesized that as microglia interact with BMVECs, the presence of the 
glial cells may influence the properties of the BBB [26]. Unfortunately, the exact mechanisms of microglia associated with the BBB have not been well characterized [26].

\subsection{The Importance of Modeling the BBB}

The most important aspect of the BBB, the selective permeability, has been the most referenced characteristic when investigating the overall properties, pathologic effects, or pharmaceutical potential of the barrier $[27,28,29]$. In terms of neurological disorders, it is thought that dysfunction of the BBB could be involved in early disease progression [11]. In Parkinson's and Alzheimer's, it is thought that the integrity of the BBB declines, resulting in decreased extraction of harmful toxins [10]. During Acquired Immune Deficiency Syndrome (AIDS) related dementia, there is an increase in leukocyte transport across the barrier [10]. Lastly, a study focused on patients diagnosed with epilepsy indicated that an increase in barrier permeability my lead to further progression of the disorder [30].

Modeling the BBB can improve not only the understanding of the progression of certain pathologies, but also its progression in the pharmaceutical industry. High throughput screening (HTS) accelerates the assessment of the toxic effects of millions of compounds to determine which would be good candidates for further investigation [31]. Permeability experiments evaluate the transport of drug candidates to the brain and their success is necessary for drug companies to progress further through drug development [32]. However, as HTS and permeability testing regimes serve as a standard approach for drug development, there is a need to improve the models of the BBB to increase the efficiency for pharmaceutical companies to create effective therapies. 
As pharmaceutical companies progress from identification and validation of a test target to compound development, they face the challenge of whether to continue the development of a new drug [32]. After undergoing HTS, researchers have indicated that $98 \%$ of early drug compounds fail to penetrate the BBB [32]. Failure to penetrate the barrier results in additional drug development, requiring additional time and money. This scenario described throughout literature and industry, reinforcing the need for more efficient models of the $\mathrm{BBB}$ whether it is in vivo, ex vivo, in vitro, in silico, or a combination of several or all of these [33].

\subsection{Approaches to Modeling the Blood-Brain Barrier}

\subsubsection{In Vivo Models}

In vivo models consist of intact living specimen, rather than a section of or a dead specimen. In vivo studies are known to be the most reliable due to the their ability to represent the brain's complex microenvironment and the advantage that the specimen's overall systemic effects can be taken into consideration $[34,35]$. Studies using in vivo approaches are conducted on both healthy and diseased models [33,34]. Analyzing permeability of the barrier can be performed using intravenous administration [33]. Different methods of intravenous administration have been developed including brain perfusion, microdialysis, and positron emission tomography [36,37].

Brain perfusion involves retrograde catheterization and ligation of the external and internal carotid arteries [36]. Permeability is quantified by looking at the uptake of the functional tissue of the brain. Brain perfusion however, is also known to be a more difficult procedure. A more novel approach, microdialysis, is being applied to measure 
influx and efflux measurements [37]. In this method, a probe is inserted into the brain to measure the extracellular fluid, which in animals can be sampled repeatedly or continuously. The invasiveness of microdialysis does become an issue however. Subsequent probe calibrations and reentry disrupt the tissue and cause variable data $[36,37]$.

Positron emission tomography (PET) has been implemented in in vivo studies for its non-invasive methods, and can be applicable to humans. PET uses an intravenous injection of a radiotracer that can be viewed under a positron-emitting scanner $[38,36]$. What is unique about PET is the ability to assess permeability of the barrier and drug distribution in specific regions of the brain. The limitations of PET stems from its low resolution between injected substances and metabolites, and high costs associated with its instrumentation [38].

In vivo $\mathrm{BBB}$ studies have progressed the research of many different fields including brain metabolism, disruption, transport, disease progression, and drug biodistribution. In vivo models require live animals that raise the ethical dilemmas of animal testing. These models are costly to obtain and also to properly house, feed, and dispose of the animals after experiments have been conducted [38]. Therefore, in vivo results are also not always translatable to humans $[39,40]$.

\subsubsection{In Situ Model}

In situ modeling focuses on studying samples while they are in their natural environment. In situ approaches also investigate healthy and diseased models [39]. In situ BBB experiments typically involve rats or porcine models $[41,42]$. A model known as single pass injection involves the injection of a single bolus of radiolabeled compounds 
into the carotid artery and allows for passage into the cerebral capillaries [40]. This single pass injection technique, or Brain Uptake Index (BUI), involves an injection of radiolabeled test and reference compounds to analyze composition modifications of the injected substance including osmotic pressure, $\mathrm{pH}$, and protein binding [36]. Single pass injections are limited in time from when the injected bolus travels through the cerebral capillaries and is subsequently lost to the systemic system in 5-15 seconds [38]. This method is also known to exclude systemic metabolism [37].

\subsubsection{Ex Vivo Models}

Ex vivo models involve experimentation on tissue in an artificial environment outside the organism. Blood-brain barrier ex vivo methodologies involve using living tissue outside of the specimen, while maintaining the environment that the tissue originates from. Ex vivo studies present the ability to study structure and function of the barrier, along with the overall environment that the brain is subjected to. Samples taken from the brain vary between acute and organotypic culture [44]. Acute studies allow for short-term (within hours) experiments [44] while oraganotypic slices involve continuing cultures in a bench top model to keep the samples alive, allowing for longer-duration experiments [45]. Both methods provide insight into the characteristics and modulation of molecular pathways, screening of therapeutic molecules, and discovery of new genes $[44,45]$.

One of the more favorable characteristics of ex vivo BBB models is the ability to only utilize a sample of the specimen without the wasting of resources seen in in vivo methods [45]. In efforts to not exclude the study on humans, ex vivo studies can be performed post-mortem, allowing extraction of the brain tissue during autopsy, greatly increasing 
the knowledge of human brain tissue [46]. However, the quality of human brain tissue, key to the validity of the studies, is greatly affected by degradation and storage temperature [46]. The degradation of certain components such as structure and proteins of post mortem specimens raise doubt whether or not conclusive results can be accepted by researchers in the field [47].

\subsubsection{In silico Models}

An in silico model typically refers to computer aided models. In silico models are typically predictive models based on in vivo experimental data [48]. These computational models provide a cheaper less restricted methodology when compared to in vivo and ex vivo approaches. There are different computational approaches to assess either permeability or pharmacokinetic parameters for BBB research [49,50,51]. The latter uses Quantitative Structure Activity Relationship (QSAR) approaches, which look at brain and blood partitioning at steady state described as $\log B B$ [50]. $\log B B$ is described as the ratio of drug concentration in blood and brain at steady state [49]. A study performed by Chen et al. utilized an artificial neural network for predicting permeability of the BBB [52]. Their investigation involved eight computational inputs of chemical and molecular components including, a multidrug resistance protein ( $\mathrm{P}-\mathrm{gP})$, transporter probability, molar refraction, and plasma protein-binding ratio to achieve an output of $\log B B$. $\log$ $B B$ has drawbacks including the requirement to measure at steady state, altering the estimate of brain uptake [52]. In silico models deviate from conventional physical models as they are based on computational models. Due to these limitations, any molecule confirmed by the model has the potential to lead to false results. 


\subsubsection{In vitro Models}

In vitro studies permit the evaluation of both animal and human cells in a simpler, reproducible, and scalable manner. Interest in in vitro models has increased due to the capabilities for predicting penetration of novel drug therapies, evaluating dysfunction of the barrier due to progression of various neuropathologies, and pre-screening and optimizing new therapies [43]. In terms of retrieving information, in vitro models present the ability to test for multiple variables at the same time with a large range of cell types.

For all types of in vitro models, each must be validated based on certain characteristics seen in vivo including tight junctions between endothelial cells, low polar molecule permeability, selective permeability based on composition, asymmetric permeability to ions, or expression of active extrusion proteins [43]. Above all, there is a desire for in vitro models to have straightforward culture protocols and for results to be reproducible.

Culture methods vary between static and dynamic in vitro systems both of which provide insight to BBB expression and capabilities [53]. Static monodimensional transwell models utilize BMVECs derived from various animal and human sources, attempting to show phenotypic characteristics seen in vivo [54]. Transwell models consist of inserts that provide compartments that are separated between permeable membranes. Cells are cultured on permeable membranes and in growth media designed for the cell types used [54]. Isolation of such cells is seen to be the more challenging aspect of the method as BMVECs are harvested from brain microvessels by mechanical and enzymatic means including homogenization, filtration, sieving, centrifugation, or collagenase [53]. Isolation of microvessels translates to disassociation from other cell types of the NVU, and loss of BBB phenotype. Consequences of such isolation lead to lack of expression of 
specific transporters, decrease in TJs, and loss of polarity of the luminal and abluminal membranes. As transwell models do not separate between luminal and abluminal membranes, the exposure of the same media to both sides may affect the structure of the cultured BBB and its characteristics [53].

Co-cultures of the barrier serve to introduce other cell types of the NVU, such as glial cells, to increase in vivo representation, expression of certain endothelial markers, trans-endothelial electrical resistance (TEER), and transport related processes [53]. TEER is a measurement used to indicate the formation of tight junctions in vitro [54]. Recent work by Hatherell et al., focused on using cultures with three associated cell types to investigate increases in TJs in human cerebral microvascular endothelial cells under the assessment of TEER (Fig. 3) [55].

Although static culture methods such as transwell inserts have the potential to introduce more BBB components, these models still lack the interaction of blood and dynamic flow characteristics that may also enhance the integrity of the BBB. 


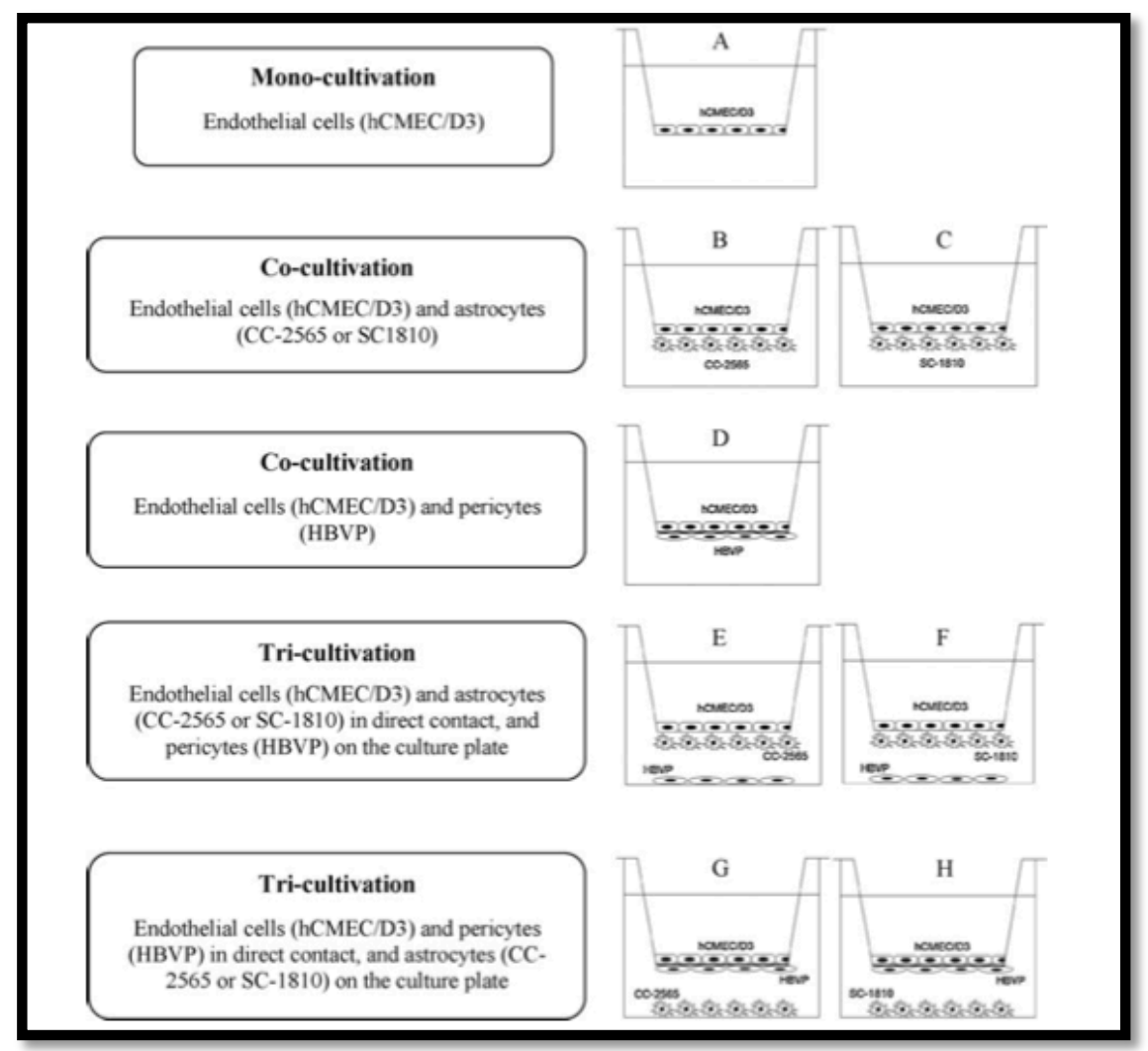

Figure 3: Schematic of work performed by Hatherell et al., evaluated three static culture models using TEER to assess tight junction resistance [55].

Dynamic culture methods utilize the characteristic of flow to model the mechanical stimuli found in capillaries with the goal of recapitulating the brain's microvasculature environment. The paradigm of such an apparatus typically involves the interaction of ECs intraluminally and a component of the NVU extraluminally in a hollow fiber polymer scaffold either made up of poly(lactic-co-glycolic acid) (PLGA) or polypropylene, among others [53]. Dynamic in vitro models of the BBB (DIV-BBB) aim to mimic the brain microvasculature, producing quasi-physiological conditions for culture of human or non-human BMVECs and other NVU cell types [56].

The potential of dynamic culture methods is not limited to implementation of hemodynamic characteristics. Introduction of a co-culture of cells is also possible, which 
allows better expression of BBB characteristics including ion channels, glucose transporters, and enhanced restrictive permeability through paracellular pathways [57] (Fig. 4).

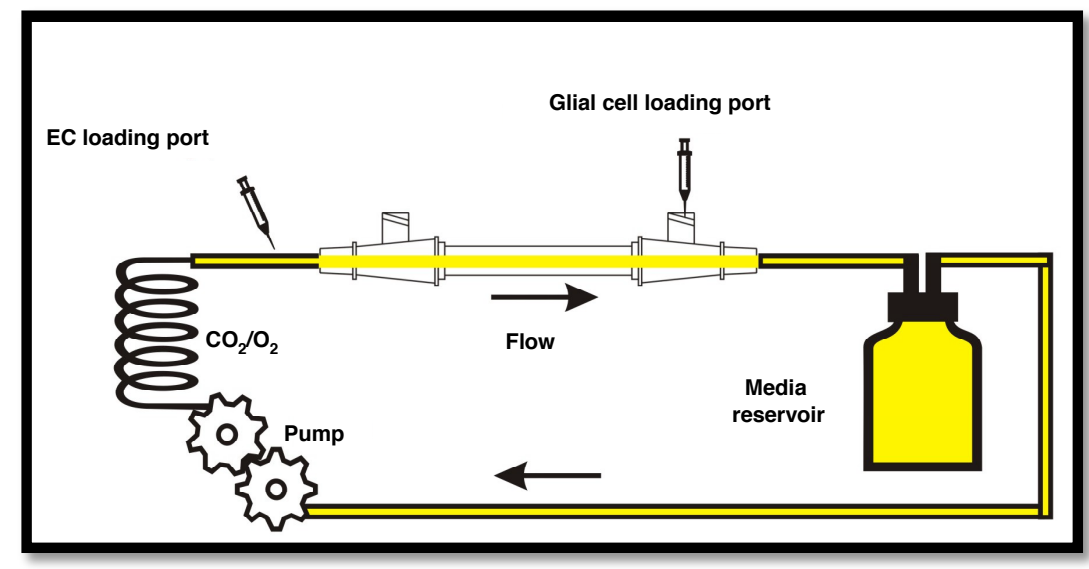

Figure 4: Schematic of one dynamic culture method utilizing a bioreactor with a media reservoir, pump, and gas exchanger [56].

One of the earlier models of a hollow fiber apparatus, developed by Cellco Inc., provided artificial capillaries made from polypropylene sealed in a chamber that was connected by gas permeable tubing [53] (Fig. 5).

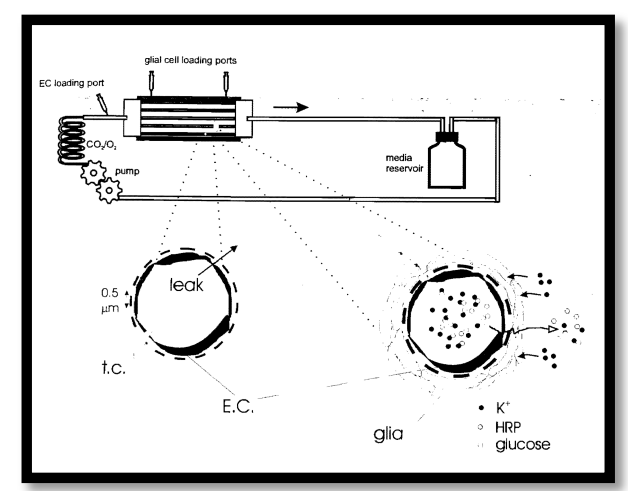

Figure 5: Cellco Inc. hollow fiber bioreactor equipped with polypropylene artificial capillaries coated with ProNectin ${ }^{\mathrm{TM}} \mathrm{F}$ [53].

Spectrum Labs then refined this particular design and improved upon scale and cell integration. Researchers who have focused on dynamic in vitro models, have often used 
the CellMax Duo system from Spectrum Labs to investigate the BBB $[53,57,58,59]$. The Spectrum system contains a perfusion pump that produces flow to a plastic bioreactor chamber containing fifty polypropylene hollow fibers [57]. Gas exchange occurs through the use of gas permeable silicone tubing [57]. Once flow passes through the outlet of the bioreactor, fluid reaches the media reservoir only to be pumped back into the system [57]. However, researchers have described the limitations of the system to include inability to observe cells, non-reusability, and compatibility issues with tissue slice preparation [58]. To advance through these limitations researchers have created their own custom bioreactor to proceed forward in BBB research (Fig. 6) [57,58].

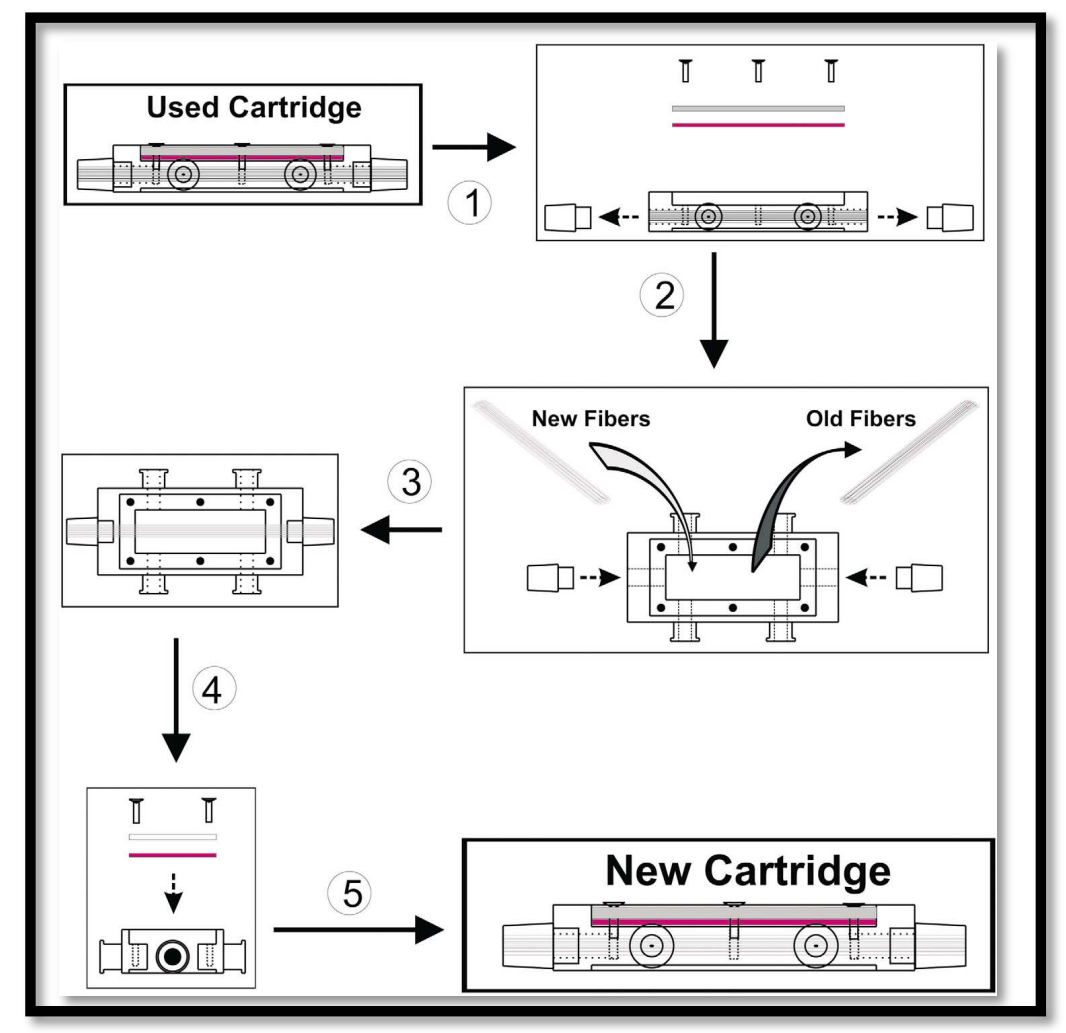

Figure 6: Custom bioreactor developed by Cucullo et al., after facing limitations with the CellMax system [58]. 
Cucullo et al. performed a study that evaluated the bioreactor shown in Figure 6 where certain design requirements were implemented and tested including that the system be reusable and allow for fiber extractions and tissue preparations [53,58]. Although different in design, the new bioreactor utilized the same methods to establish flow seen in the CellMax system. The design of this bioreactor addressed the limitation of single timepoint evaluations seen in the CellMax system by the use of a removable window [58]. This resulted in sequential removal of the artificial capillaries without affecting the remaining fibers that were not intended for analysis [58]. These researchers were able to develop a model of the BBB to characterize co-cultures of endothelial and glial cells [60].

\subsection{Bridging the Gap Between In vivo and In vitro Models}

When comparing all of the available models of the BBB, the capabilities of the most translatable method, in vivo, and most scalable, in vitro, may be combined to produce an improved model of the BBB. Taking the information gathered from in vivo, the $\mathrm{BBB}$ can be reverse-engineered in vitro. With a focus on dynamic in vitro models, there is the potential to introduce multiple cell types of the NVU and hemodynamic forces to enhance the characteristics seen in the native BBB. This could also produce a cost effective model and allow for accelerated results that can be directly applicable to humans. Researchers in the field have been progressing toward the development of more robust models of the barrier for toxicological screening, drug permeability, and general characterization. Dehouck et al. conducted a study evaluating the correlation between in vitro and in vivo models [61]. Dehouck used an in vivo intracarotid injection model to compare drug concentration to an in vitro co-culture BBB model utilizing BMVEC and 
astrocytes and showed a strong correlation between the drug concentrations for the two models [61]. This study suggests that in vitro models can yield appropriate results compared to in vivo to further develop a BBB.

\subsection{Academia's Contribution}

Currently, the Tissue Engineering Laboratory directed by Dr. Kristen Cardinal at California Polytechnic State University, San Luis Obispo performs research in the use of blood vessel mimics. Using various scaffold types including expanded polytetrafluoroethylene (ePTFE) and PLGA, students apply cell culture methods of sodding cells into the scaffolds to create tissue engineered blood vessel mimics for coronary device testing. This tissue-engineering paradigm can be applied to model the BBB as well, on a smaller scale. The technique of using scaffolds as a support for ECs and astrocytes can be applied for the development of an in vitro BBB. With the use of bioreactors, the lab can introduce culture protocols to in vitro models of the BBB that can help develop solutions to impact the drug discovery process.

Previous work performed by Bryan Brandon evaluated the use of one commercial system in particular to establish an in vitro model of the BBB [62] (Fig 7). Mr. Brandon's accomplishments led to the development of a protocol for the previous model of the BBB in the tissue-engineering laboratory. Mr. Brandon's first aim was to establish cell culture protocols for both bovine aortic endothelial cells and C6 glioma cells, which was completed in culture flasks [62]. The second aim was the development of an in-house bioreactor that would improve upon the commercial system [62]. The third aim was to facilitate the co-culture and analysis of the BBB in vitro model [62]. Later work from Ian Mahaffey's senior project contributed in the initial design of the bioreactor discussed in 
this thesis. In addition, he created a mandrel to collect electrospun fibers, and evaluated scaffolds for use in a BBB model [63].

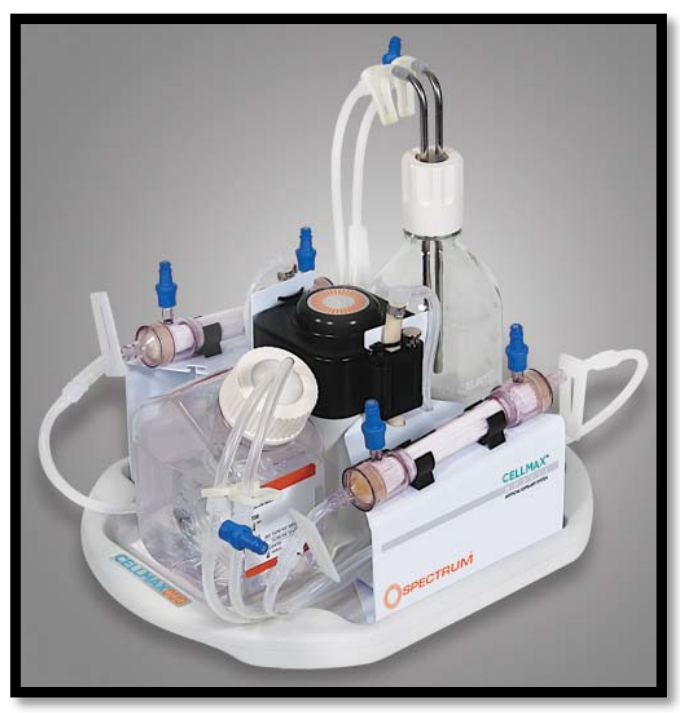

Figure 7: CellMax Duo Bioreactor system characterized by Bryan Brandon in the Cal Poly Tissue Engineering Lab [62].

The commercial system utilized in Mr. Brandon's project, the CellMax Duo Hollow Fiber Cell Culture System, was equipped with a hollow fiber bioreactor that consisted of sixty polypropylene fibers with $330 \mu \mathrm{m}$ inner diameters [60,64]. The system also included a perfusion pump with positive pressure displacement capabilities that could assist the growth of two bioreactors simultaneously [64]. The element of flow was developed from the use of various Teflon pump bars that compressed the pump tubing and allowed flow from the media reservoir to the bioreactor [64]. Sampling and interaction of the extra-capillary space was accessible through transluminal ports on the side of the bioreactor. To access the scaffold, destruction of the bioreactor was required with the use of a bandsaw to cut through the epoxied ends holding the polypropylene fibers. Attempts to retrieve the fibers were possible; however, after immersing the scaffolds in fixative, degradation occurred and the loss of data created the need to pursue 
the second aim in Mr. Brandon's initial goals: to create a bioreactor that would improve upon some of the limiting characteristics of the commercial system.

A new design for an in-house bioreactor had to decrease the costs of the overall system, reuse the system, and allow for users to retrieve the scaffolds after cultivation for further assessment. The design of the bioreactor was developed so that alterations to the bioreactor could be made in-house. In terms of sterilization, the design had to be compatible with techniques available including autoclave and ethylene oxide (EtO). The bioreactor also needed to be adaptable to use the PLGA that was currently made inhouse, the ePTFE used in other divisions of the lab, or possibly decellularized vessels.

\subsection{Summary of Overall Thesis Goals}

Based on the need for appropriate in vitro BBB models and the preliminary work by Bryan Brandon and the Cal Poly Tissue Engineering lab, the overall goal of this thesis was to develop and characterize an in-house bioreactor for the cultivation of a tissueengineered blood brain barrier, which met the following requirements.

The first aim was the design and development of a bioreactor chamber and system that could be used to cultivate the BBB. Design criteria were based on the advantages as well as the limitations of the CellMax system and the specific needs of the laboratory. The goal was to create a bioreactor that was reusable, capable of being sterilized, able to be monitored, easily used by students trained in the laboratory, and developed so that scaffold extraction is possible.

The second aim was the characterization of flow and capabilities of the custom bioreactor. This involved analyzing flow of the bioreactor using the pumping apparatus from the CellMax system, and characterizing the Teflon bars to determine which was 
optimal for physiological flow conditions. Capabilities of the system that needed to be characterized included assembly of the bioreactor, packaging for sterilization, and overall use during cultivation. This involved testing the bioreactor under sterile and non-sterile conditions, including multi-scaffold and long-term cultivation.

The third and final aim was to develop protocols for the overall use of the bioreactor to ultimately allow co-cultures of BAEC and C6 glioma cells and advance the creation of an in vitro model of the BBB. This involved establishing protocols for assembly of the bioreactor, insertion of scaffolds, cell preparation prior to integration of the bioreactor, cell sodding, and system incubation. Each of these aims will be addressed in the subsequent chapters. 


\section{Chapter 2: Initial Design of BBB Bioreactor, Manufacturing, \& Costs}

\subsection{Introduction}

DIV-BBB has been of interest to researchers for over a decade, with many researchers specifically focused on hollow fiber apparatuses $[53,57,58,59]$. Regardless of how the model is constructed, there are certain requirements that a BBB model should have. An in vitro model of the BBB must be able to allow expression of tight junctions between ECs, which translates to having a restrictive permeability through the paracellular and transcellular route [65]. To achieve this, the correct flow conditions, $\mathrm{pH}$, and temperature must be maintained to encourage such characteristics.

The commercial system that was previously used in the tissue-engineering lab, the CellMax Duo from Spectrum Labs, provided researchers with models of the BBB that expressed low permeability to intraluminal ions, low extravasation of proteins, and low expression of glucose transporters [58]. However, analyzing the hollow fibers was difficult: destruction of the bioreactor was the only solution to gain access to the fibers, and these fibers were only compatible with certain sectioning techniques [57,58].

Thus there is a need for a new bioreactor design to recapitulate the BBB in a costeffective and user-friendly manner. The design requirements were as follows: the bioreactor should be made in-house with the resources available at the university, at a low cost, reusable, sterilizable using methods available on campus, and allow for scaffold extraction. 


\subsection{Initial Designs of the BBB Bioreactor}

Development of a new bioreactor to model the BBB started with initial designs that were based on design criteria and limitations of the current model used in the lab. Ideas were recorded and drawn into a design notebook before development in a 3D modeling program titled SolidWorks (SolidWorks Student Design Kit 2010). Two major initial designs were generated, and are described in detail below, including discussion of individual parts and overall functionality.

\subsubsection{Design 1}

The first design originated from Dr. Kristen Cardinal's Biomaterials class with the help of Erik Schulter, Paige Ryan, and Kelly Roberts. Mr. Brandon's second aim was reiterated to the class for this project, with the goal of finding an alternative design to alleviate the problems seen in the CellMax system. A new design was developed using SolidWorks providing a three dimensional model consisting of a cartridge system with two end caps aligned together by two connection bars, an outer housing with two transluminal ports, and feeding caps for the inlet and outlet (Fig. 8). 


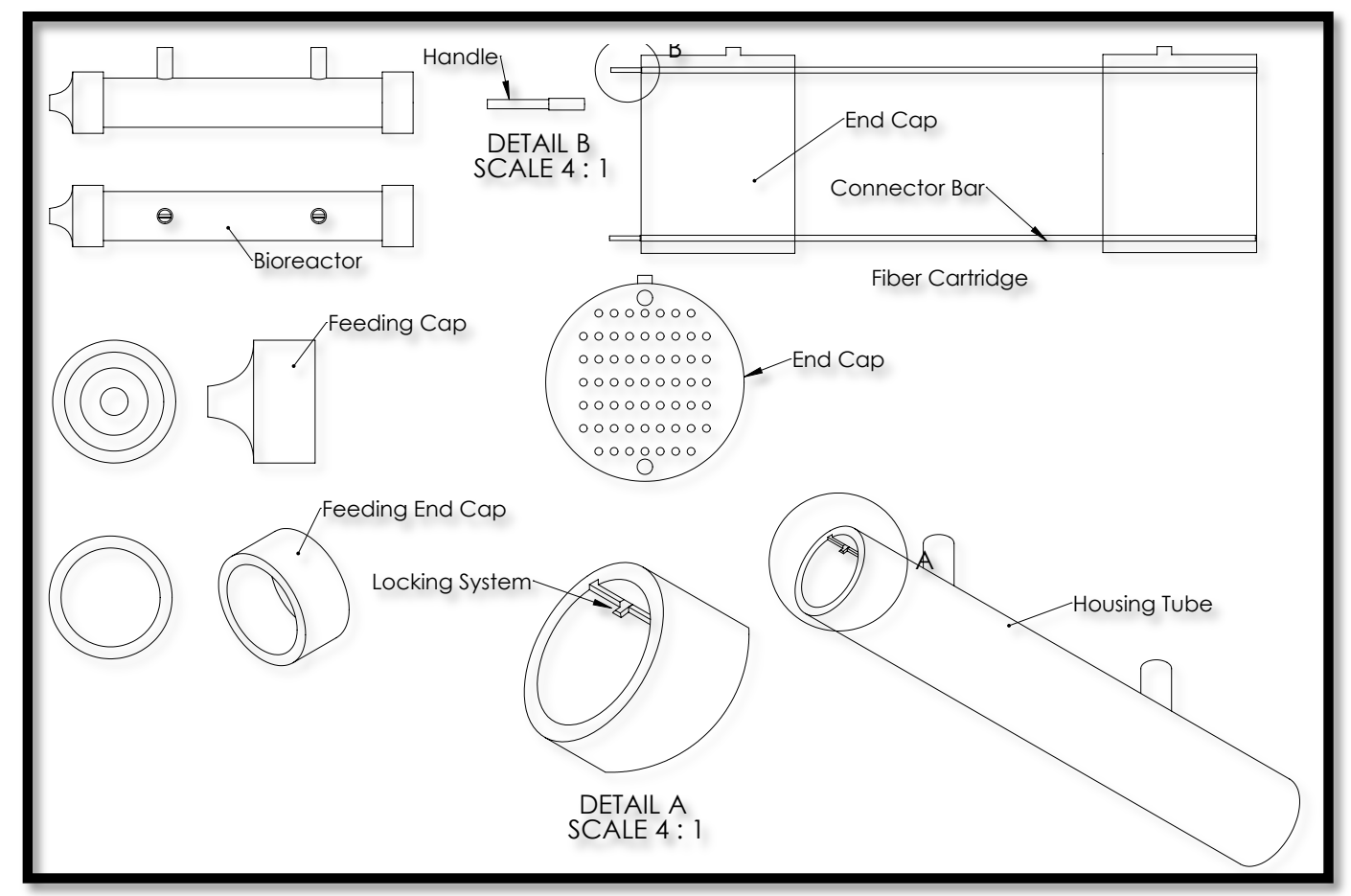

Figure 8: SolidWorks drawing of the first design of the bioreactor. Drawing includes all of the components and features including the locking system for the cartridge. Designed and drawn in SolidWorks by Amin Mirzaaghaeian.

The end caps could potentially allow organized scaffold arrangement and alignment, which was also facilitated by the connector bars. The assembly of the two feeding end caps with the connector bars formed the cartridge that would be inserted to the outer housing. The outer housing was to be manufactured out of a transparent material to allow visibility of the bioreactor during its culture period. Transluminal ports were integrated to permit sampling of the extraluminal fluid, alleviate pressure build up, and allow pressure sodding of cells. The outer housing also included a channel that ran along the longitudinal axis on the inner diameter of the cylinder, which guided the cartridge through the outer housing. Once the cartridge was fitted into the cylinder, it would be fastened when twisted counter clock wise into notches, located at the tail ends 
of the outer housing, perpendicular to the longitudinal channels (Fig. 8). Feeding end caps were then installed onto the ends of the outer housing for induction of media at the inlet. After approval from Dr. Cardinal, a project was created to further develop the design and manufacture the new bioreactor.

\subsubsection{Evaluation of Design 1}

The design was brought to David Laiho to assess design and manufacturing options. His assessment concluded that the inner locking mechanism was impossible to manufacture if the housing was to be made in one piece. In terms of securing the scaffolds, the scaffold cap provides holes where the scaffolds can be glued. However, for the scaffold caps to be reusable, securing the scaffolds with glue would make it difficult for multiple uses. To proceed with this scaffold cap design would require finding a medical grade adhesive that had a complementary solution to degrade the glue for installation of new scaffolds. When installing transluminal ports, machining a hole on a cylindrical surface raised possible leaking issues. To proceed with this idea, he suggested machining the hole and glue around the component that would represent the port, or install an O-ring around the component to prevent leaking. Furthermore, Mr. Laiho suggested keeping the cartridge, but to develop an alternative way to secure the cartridge inside the outer housing. After reviewing the issues of securing the cartridge and scaffolds, sealing the bioreactor, and including transluminal ports, Mr. Laiho's assessment led to the decision to design a new bioreactor. The overall evaluation of the first design is summarized in Table I below. 
Table I: Design evaluation for initial design 1

\begin{tabular}{|l|l|l|}
\hline & Result & Comments \\
\hline Manufacturing & Complicated & Locking system impossible \\
\hline Cost & Variable & Material dependent \\
\hline Ease of Use & Yes & Cartridge reduces total parts \\
\hline Reusable & Yes & $\begin{array}{l}\text { Sterilization procedure } \\
\text { dependent }\end{array}$ \\
\hline Sealing & No & Friction not enough to seal \\
\hline Multiple Scaffolds & Yes & Organized arrangement \\
\hline
\end{tabular}

\subsubsection{Design 2}

Based on the evaluation of the first design, the second design of the bioreactor included several of the same components, but differed in the design of each component. The initial cartridge idea was kept with a design that would allow the cartridge to be pushed through an outer housing (Fig. 9).

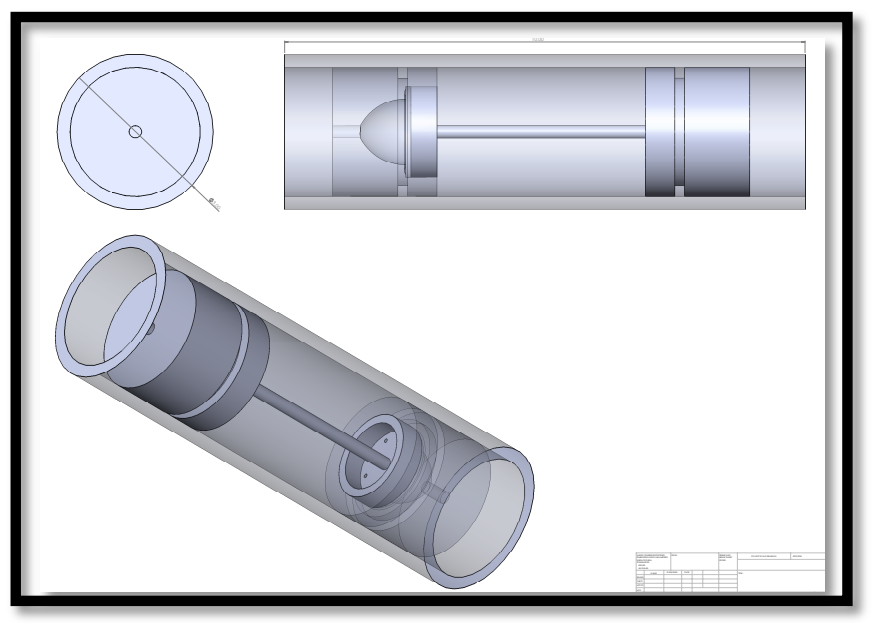

Figure 9: Solid drawing of cartridge designed to be pushed through the outer housing. Drawn by Amin Mirzaaghaeian. 
The cartridge was composed of two scaffold caps stabilized with a rod that would fit in the middle of each cap creating a dumbbell configuration. Each scaffold cap would have eight installation ports for scaffolds to be glued into. Once the scaffolds and stabilization bar were fitted to each scaffold cap, the scaffold caps would be fitted into two outer caps that would complete the cartridge configuration. The two outer caps would allow flow through the inlet and outlet to the media reservoir. Sealing of the bioreactor would be achieved by introducing an O-ring groove on the outer diameter of the outer caps. Once the cartridge was fitted with O-rings it would then be pushed through the inner diameter of the outer housing until both sides of the cartridge was secured inside. Transluminal ports were developed with holes machined on the outer diameter of the outer housing, and fittings to be screwed in with an O-ring fastened around it to prevent leaking.

\subsubsection{Evaluation of Design 2}

Design 2 overcame the limitations seen in Design 1 with the implementation of an improved cartridge apparatus that would allow easier scaffold installation and sealing of the bioreactor. With a larger outer housing diameter, the O-rings fastened around the fittings were thought to be adequate to prevent leaks. Although the new cartridge design was sufficient to seal the contents of the bioreactor, having to extract the scaffolds at different time points was not a function included in this design. The method of pulling the cartridge out, replacing the media, and avoiding contamination would be very complicated. Further development was needed to complete the design of the bioreactor. The table below describes the evaluation of Design 2. 
Table II: Design Evaluation for initial design 2

\begin{tabular}{|l|l|l|}
\hline & Result & Comments \\
\hline Manufacturing & Possible & CNC or Lathe \\
\hline Cost & Still Variable & Material dependent \\
\hline Ease of Use & Yes & Cartridge reduces total parts \\
\hline Reusable & Yes & $\begin{array}{l}\text { Sterilization procedure } \\
\text { dependent }\end{array}$ \\
\hline Sealing & Yes & $\begin{array}{l}\text { O-ring grooves used for } \\
\text { sealing }\end{array}$ \\
\hline Multiple Scaffolds & Yes & Organized arrangement \\
\hline
\end{tabular}

\subsection{Design 3}

This was developed using the ideas of the second design, but altering the outer housing to be equipped with a detachable window for scaffold extraction. In addition, plans to glue scaffolds into the scaffold caps was rejected, and suturing the scaffold onto barbs was chosen. To seal the window and to prevent leaking, silicon rubber would be implemented around the edges of the window. The design of the bioreactor included two scaffold caps, a stabilization bar, two outer caps, bioreactor cap, and an outer housing. At this stage of development, each part was modeled and dimensioned in SolidWorks (Fig. 10). Complete solid drawings can be referred in Appendix B. The next step was to find the most efficient method for manufacturing that would fulfill the requirement of building the bioreactor in-house. 


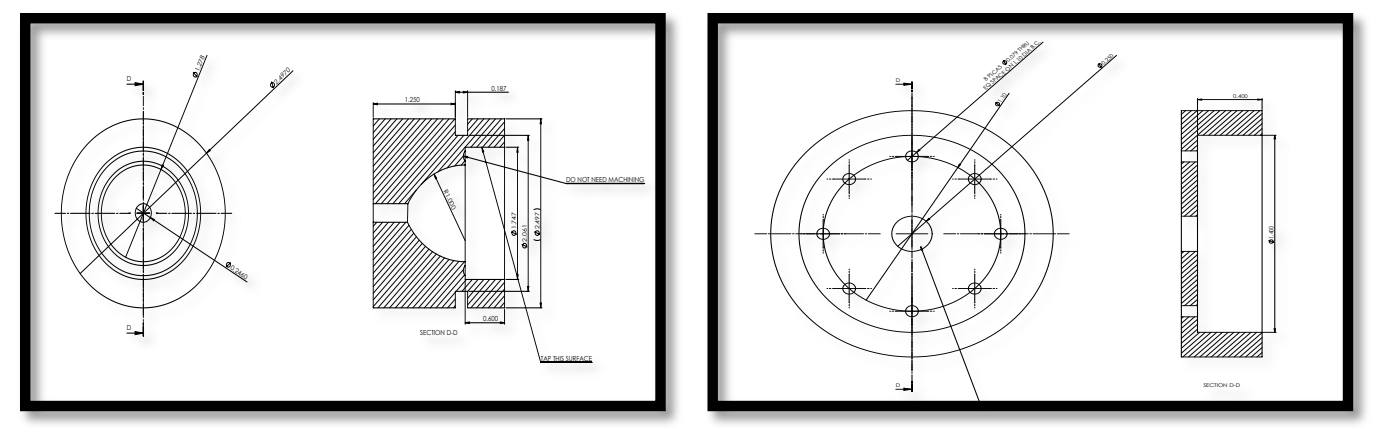

Figure 10: Solid Drawing created prior to manufacturing with all features dimensioned and specified.

\subsection{Manufacturing}

Campus resources used to manufacture the bioreactor included rapid-prototyping and machining methods. Rapid prototyping was first explored with the plan of utilizing the Stratasys systems used in the Biomedical Engineering Department. The advantage of using this system was the short time it would take to manufacture each of the parts; the components were estimated to take a couple of days to complete. This method of manufacturing was rejected after discovery of the material used and the system compatibility issues of the Stratasys system. The material used, P430 ABS plastic, was mentioned only for creation of prototypes and not considered biocompatible. A focus was then directed toward other Stratasys products to determine if they supplied materials used for medical devices. ABS-M30i and PC-ISO (polycarbonate-ISO) were two materials found suitable for biocompatibility that could also be EtO sterilized. Unfortunately, the cartridge that supplies these materials was not compatible with our models on campus. To use these materials, the department would have needed to purchase the FORTUS system, which was not feasible. Another option for rapid prototyping included equipment in the Mechanical Engineering Department, such as the Objet printer. With the help of Larry Coolidge, the Objet printer system was investigated for use to manufacture the bioreactor. 
The Objet system was known for its higher resolution compared to other fused deposition modeling systems provided on campus. Cost for using the printer was $\$ 25$ for the job and an extra fee depending on how much resin was used. The resin used in the Object system (Eden250) was first assessed for its biocompatibility. The materials fact sheet for the resin indicated that it was not suitable for medical device use. Therefore, of the possible options for manufacturing, rapid prototyping on campus was rejected. The last option for rapid prototyping was a company here in San Luis Obispo. Alltec Inc. was found based on its connection with the Mechanical Engineering Department. Manufacturability of this design was discussed with Randal Dennis, a mechanical engineer from Alltec Inc. After the assessment that the material would need to be sterilized and medical-grade, Dennis concluded that Alltec could not fulfill those requirements and recommended machining the parts instead.

Machining was considered the final option in creating the bioreactor. A question of where to machine the parts was raised based on the limiting factors of time and money. The options were between outsourcing to a machinist and having it done by a student. Material research was required before a decision was made. This research led to selecting 316-stainless-steel based on its mechanical and material attributes. The disadvantage of this material was that it required a slow machining procedure with a coolant of either gas or water to assess heating of drill bits. As a result, a professional machinist was chosen to help assess the complex machining and to manufacture the parts. Design of the bioreactor components were finalized in SolidWorks (Appendix B) and sent for manufacturing. Two outer caps, two scaffold caps, and a stabilization bar were machined out of 316-stainlesssteel. 
The one part of the bioreactor that was manufactured in-house was the outer housing of the bioreactor. Acrylic was chosen for its transparency, ease of machining, and low cost. The acrylic outer housing was dimensioned to be 6" in length and 3" in diameter representing the overall size of the bioreactor. The Mechanical Enginnering Department's Bonderson 60 machine shop was used for creating the outer housing. Milling was used to create the window, while a bandsaw was used to create the bioreactor top, and drill press to create all the outlets (Fig. 11).

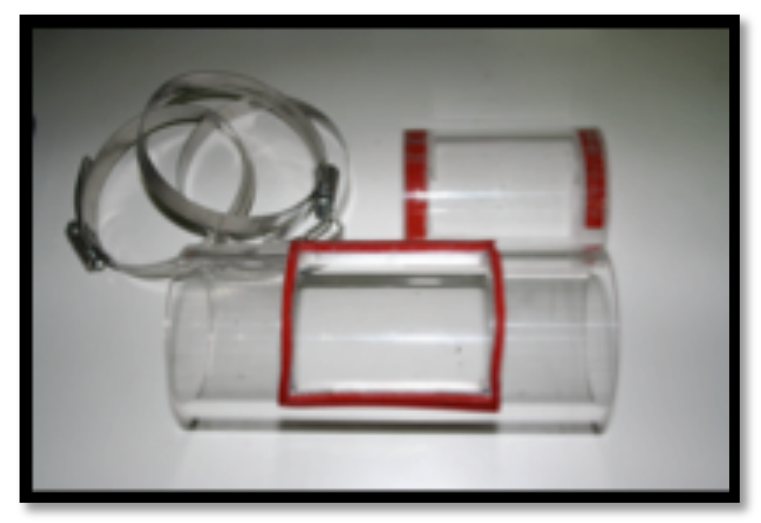

Figure 11: Outer housing machined in-house with bioreactor cap and silicon gasket material to create sealing of window

Around the edges of the machined window, silicon rubber with a durometer rating of 50A and adhesive backing supplied by Mcmaster-Carr was installed (5787T63). Perpendicular to the longitudinal axis of the outer housing in the center of the housing, two \#10-32 holes were drilled and tapped to allow fittings to be threaded onto, representing the transluminal ports of the bioreactor. 
The bioreactor cap consisted of a $3 \times 3$ " piece of acrylic manufactured from a 3.5 x 3.125" cast acrylic tube. The concave side of the cap was fitted with the same silicon rubber applied on the outer housing. The cap would be secured onto the outer housing with worn-driven hose clamps with thumbscrews. The clamps would be positioned at the ends and in the middle of the bioreactor cap.

\subsection{Machined Parts and Bioreactor Components}

\subsubsection{Outer Caps}

The outer caps represent the two ends of the bioreactor, with no directional orientation when choosing which to be the inlet or outlet. The diameters of the caps were slightly less than 2.5 ". Internally, each cap contains an inlet or outlet shown in Region I in the diagram below (Fig. 12). The inlet lumen then leads to Region II, the conical region.

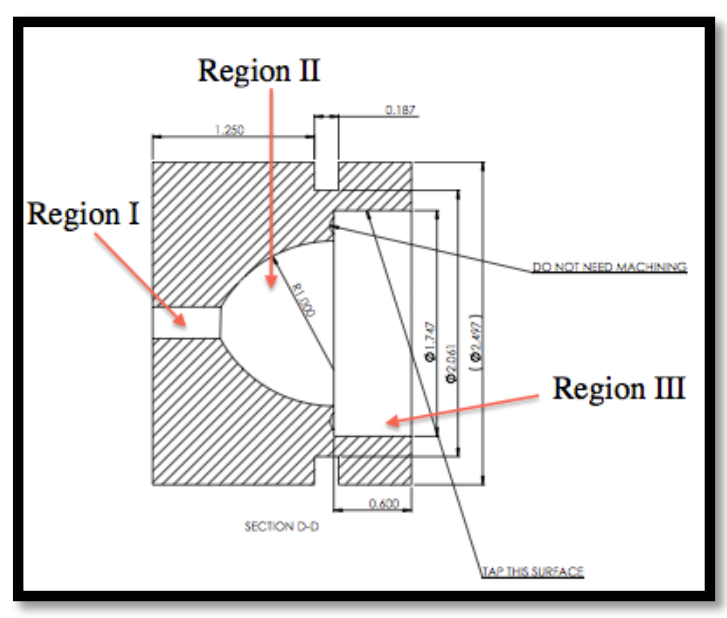

Figure 12: Schematic displaying different internal regions of the outer cap.

The design of this feature was intended to avoid turbulent flow and to provide uniform distribution of fluid to a surface. Region III represents where the scaffold cap would be threaded and secured into the outer caps. On the outer diameter of the outer 
caps, an O-ring groove was designed to allow ASM-228 silicon O-rings to sit in the groove and provide the required seal of the bioreactor (Fig. 13). Unfortunately, there was an error dealing with an incorrect reference dimension during the manufacturing process. The result of the error was a groove depth twice the amount that was originally specified based. This error was taken in account and discussed further in phase I of initial testing.

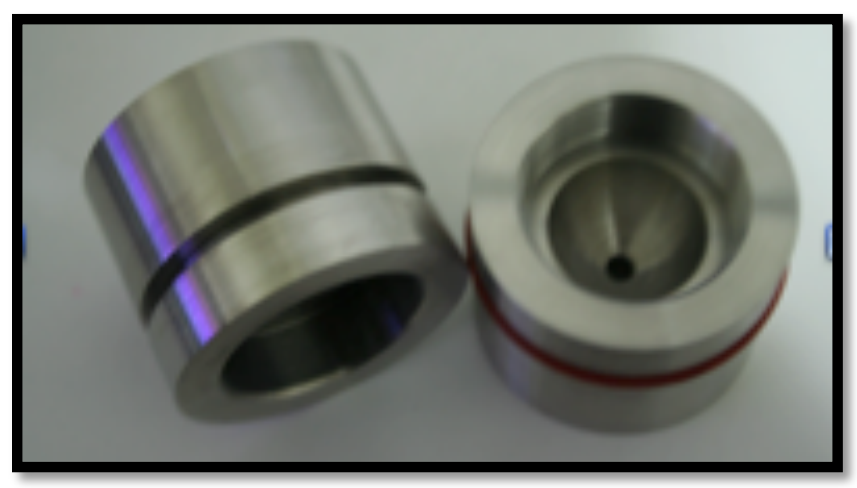

Figure 13: Machined outer caps with O-ring grooves to allow fitting of O-rings to create seal when inserted into the outer housing.

\subsubsection{Scaffold Caps \& Stabilization Bar}

This component of the bioreactor consisted of a scaffold cap with and without a stabilization bar (Fig. 14). These caps were designed to attach eight tissue scaffolds, $1 \mathrm{~mm}$ in diameter, using $2 \mathrm{~mm}$ holes machined into the caps. One of the scaffold caps was designed to allow insertion of the bar into a 0.25 " diameter hole creating the smaller "dumbbell" configuration previously mentioned (Fig. 14). This was implemented so that scaffolds could be installed on the one scaffold cap connected to the stabilization bar, and would align to fit into the other scaffold cap. The scaffold caps would then be threaded into the other caps to create the cartridge configuration that would later be inserted into the acrylic outer housing. 


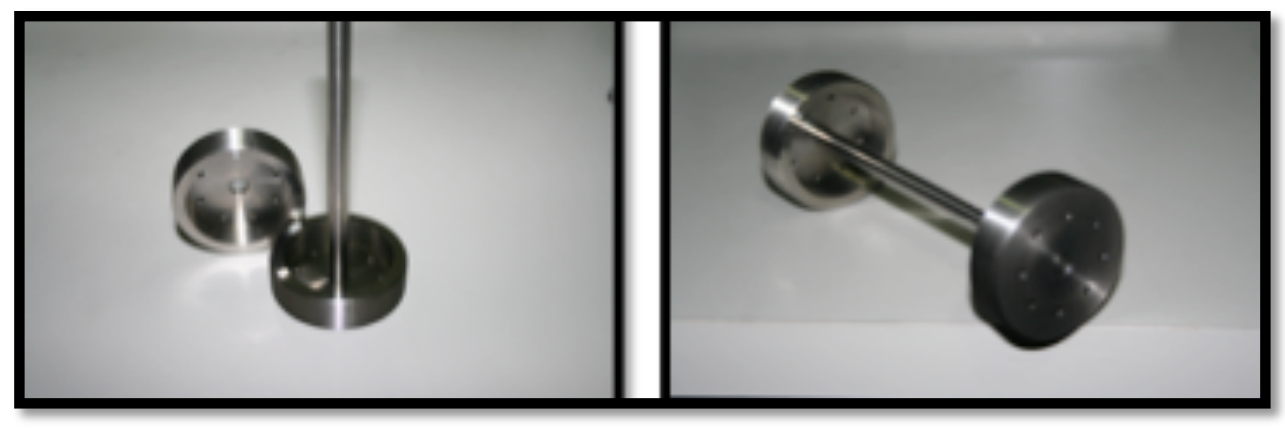

Figure 14: Machined scaffold caps with and without stabilization bar. Initial "dumbbell" configuration is achieved when insertion of stabilization bar into other scaffold cap.

\subsubsection{Scaffolds \& Barbs}

In the Tissue Engineering Lab, there are two types of scaffold material used:

ePTFE and PLGA. PLGA is electrospun in-house and serves as another division in the $\mathrm{BBB}$ research. Fibers spun are on the order of microns in the range of 2-3 $\mu \mathrm{m}$ [66]. The size of the scaffolds can be adjusted with the use of different sized mandrels. For the $\mathrm{BBB}$, the goal was to have scaffolds in the $1 \mathrm{~mm}$ range, however, based on the small scale of the fitting required for such sized scaffold, custom machining of a barb would have included additional time, money, and research and development. The actions to develop these barbs were not included in this thesis. However, $2 \mathrm{~mm}$ barbs were commercially available from Qosina, a fitting/accessory company centered in wholesale, and therefore this size was selected for the BBB scaffolds. A product sample of female luer lock to barb connector was donated to the project by Qosina, allowing the use of $2 \mathrm{~mm}$ scaffolds (Fig 15). Scaffolds were spun on a miniature 304 stainless steel 15-gauge tube (Fig. 16). 


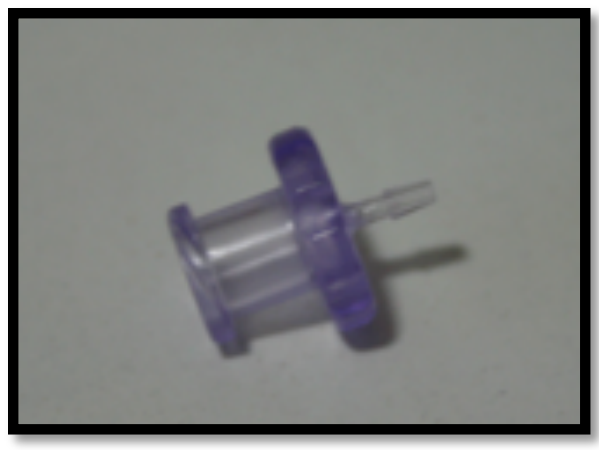

Figure 15: Qosina barb designed to fit $2 \mathrm{~mm}$ scaffolds.

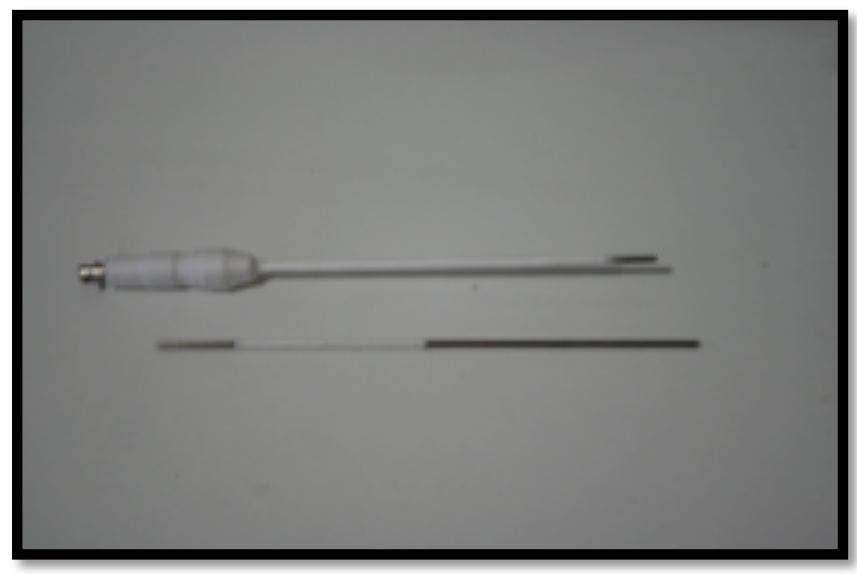

Figure 16: PLGA scaffold spun on precision miniature 304 stainless steel 15-gauge tube [66].

\subsubsection{Fittings and tubing}

Fittings for the bioreactor included one- and three-way stopcocks, Y-Connector$1 / 8$ " barbs, luer barbs, and tube clamps. Tubing included both 0.25 " silicon and 0.125 " gas permeable tubing that led the path of flow from the inlet to outlet. Female luer threaded barbed fittings and male luer lock barbed fittings were used to connect tubes and other fittings to each other. Barb to $1 / 4$ " thread fittings used to connect the 0.25 " tubing were threaded at Region I of each outer cap. End caps were used to stop flow out certain exits or during 1, 2, or 3 scaffold set-ups to block flow in any open scaffold ports. Male luer-to-thread and male-to-male collar fittings were used to connect the Qosina barbs. Tube clamps were used externally where flow needed to be stopped (Fig. 17). 


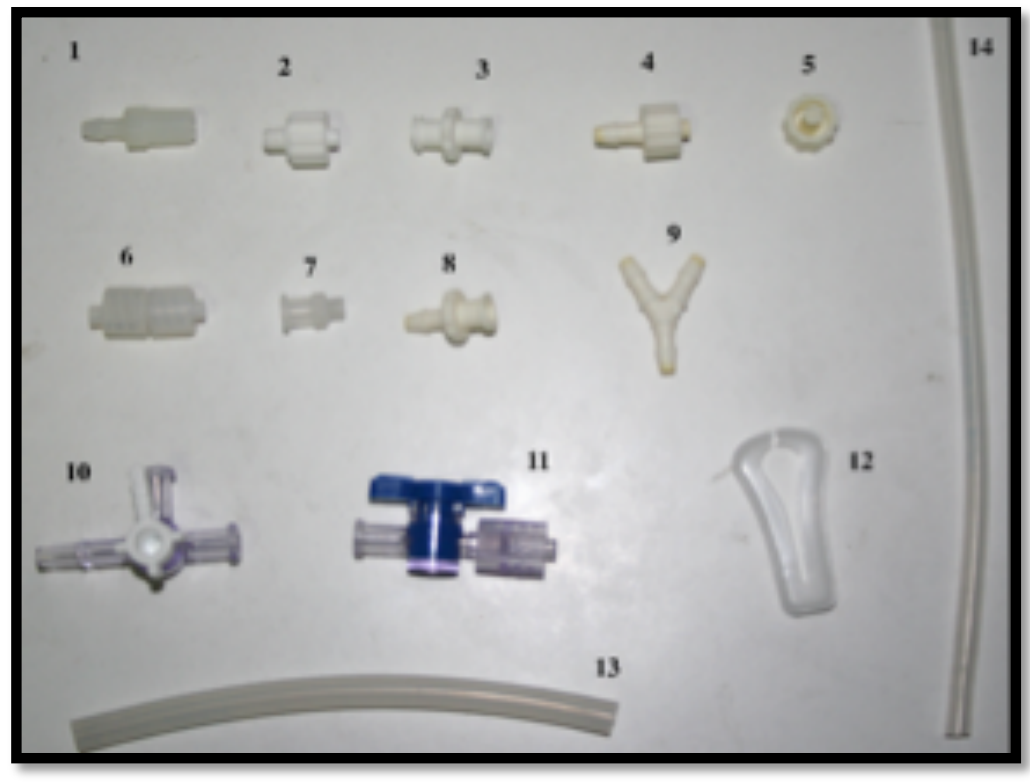

Figure 17: Diagram of all fittings used in bioreactor system except for Qosina barbs. 1.) Barb to 4-25 thread. 2.) Male luer integral lock ring to 10-32 special tapered thread. 3.) Female-female connector. 4.) Female luer thread barbed fittings. 5.) End caps. 6.) Maleto-male luer lock connectors. 7.) Female luer thread style with $1 / 4$ " hex to 10-32 UNF thread. 8.) Male luer thread barbed fittings 9.) Y-Connector-1/8" barbs. 10.) 3-way stopcock. 11.) 1-way stopcock. 12.) Tube clamps. 13.) 0.25 " silicone tubing. 14.) 0.125 " silicone gas permeable tubing. Part information is available in Appendix B.

\subsubsection{Media Reservoir \& Perfusion Pump}

The media reservoir consisted of a $50 \mathrm{ml}$ conical, which could house media

corresponding to a specific cell type. The conical cap was integrated with two holes corresponding to an inlet and outlet of the reservoir. The outlet port of the reservoir extends down to the bottom of the conical, while the inlet port was located at the reservoir cap. The extension of the outlet port was to allow the media level to decrease to the lowest amount before installing a new media reservoir. Outlet fluid from the bioreactor entered at the cap, while the oulet fluid exited at the base of the conical (Fig. $18)$. 


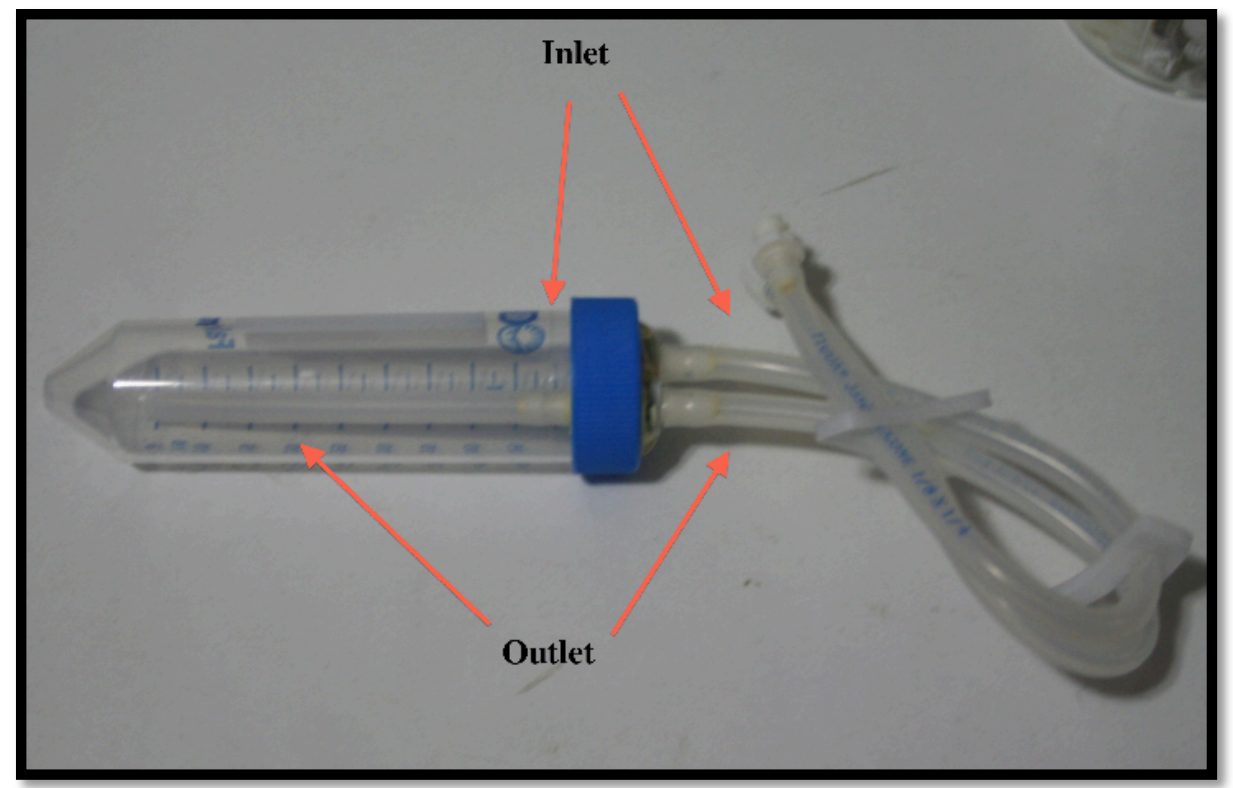

Figure 18: Media reservoir 50ml conical with inlet and outlet shown.

The perfusion pump that was used was the same pump used in the CellMax system. The pump was supplied with its own pump tubing and tubing holder that was mounted to the pumping mechanism used to produce flow. The pump was also equipped with a switch that allowed eleven modes of pump speeds (Fig. 19). For the CellMax system, each speed had been characterized and associated values of milliliters per minute were given $[62,64]$.

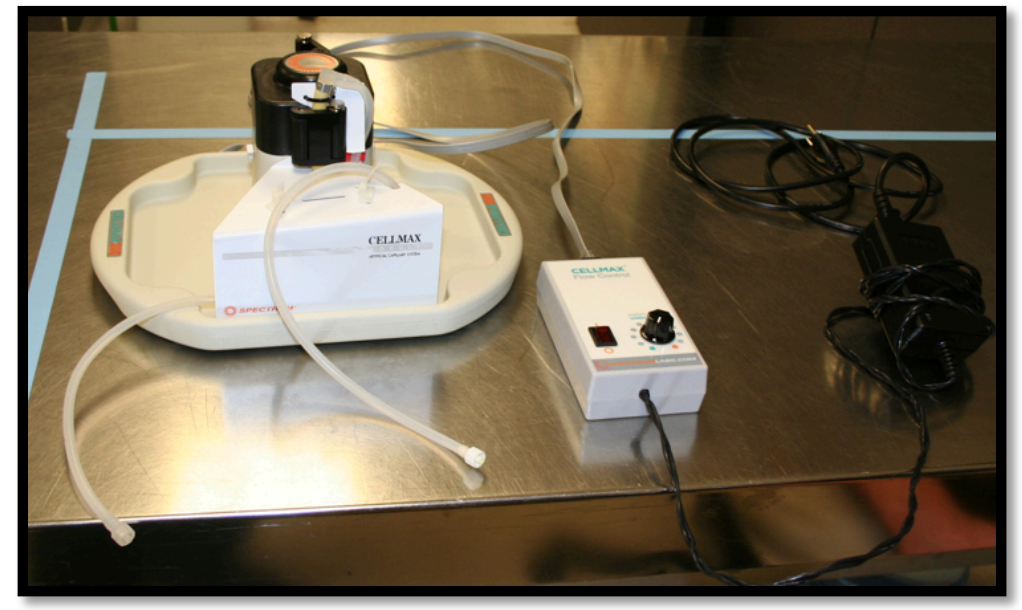

Figure 19: Pump system used during experiments that required flow. 


\subsection{Cost}

The system needed to be cost effective, especially when compared to the CellMax system, and therefore the overall cost was assessed. The cost of the bioreactor system was broken down into stainless steel components, acrylic, O-rings, tubing, and fittings. The following table presents the overall cost breakdown. Information regarding vendor, product numbers, quantity, and pricing can be found in Appendix B. This improved upon the $\$ 500$ cost for one CellMax hollow fiber bioreactor for a single use. The $\$ 200$ cost for the stainless steel parts was an up-front cost, with no maintenance fees or recurring charges.

Table III: Bill of materials with costs included showing total cost of bioreactor system.

\begin{tabular}{|l|l|}
\hline Stainless Steel & $\$ 200$ \\
\hline Acrylic Outer Housing & $\$ 34.42$ \\
\hline Acrylic Bioreactor Cap & $\$ 35.17$ \\
\hline Custom O-rings & $\$ 25.82$ \\
\hline Silicon sheet material & $\$ 34.45$ \\
\hline Custom Internal O-rings & $\$ 17.41$ \\
\hline Female Luer Lock to Barb Connector & Donated \\
\hline $\begin{array}{l}\text { Male Luer Integral Lock Ring to 10-32 } \\
\text { Special Tapered Thread }\end{array}$ & $\$ 31.82$ \\
\hline $\begin{array}{l}\text { Female Luer Thread Style with } 1 / \text { " } " \text { Hex to } \\
\text { 10-32 UNF Thread }\end{array}$ & $\$ 16.50$ \\
\hline Total & $\mathbf{\$ 3 9 6 . 5 9}$ \\
\hline
\end{tabular}




\section{Chapter 3: Initial Testing of Bioreactor}

\subsection{Initial Testing}

Once all associated parts including fittings and O-rings were purchased, and the bioreactor was constructed, initial testing of the bioreactor was performed (Fig. 20). The bioreactor was tested for leakage, the ability to be sterilized, and its ease of use. The tests performed were separated into three phases I, II, and III. Phase I testing dealt with any test protocols that involved fluids (water or media) including leak tests, flow characterization, and overall behavior of the bioreactor when internally exposed to fluids.

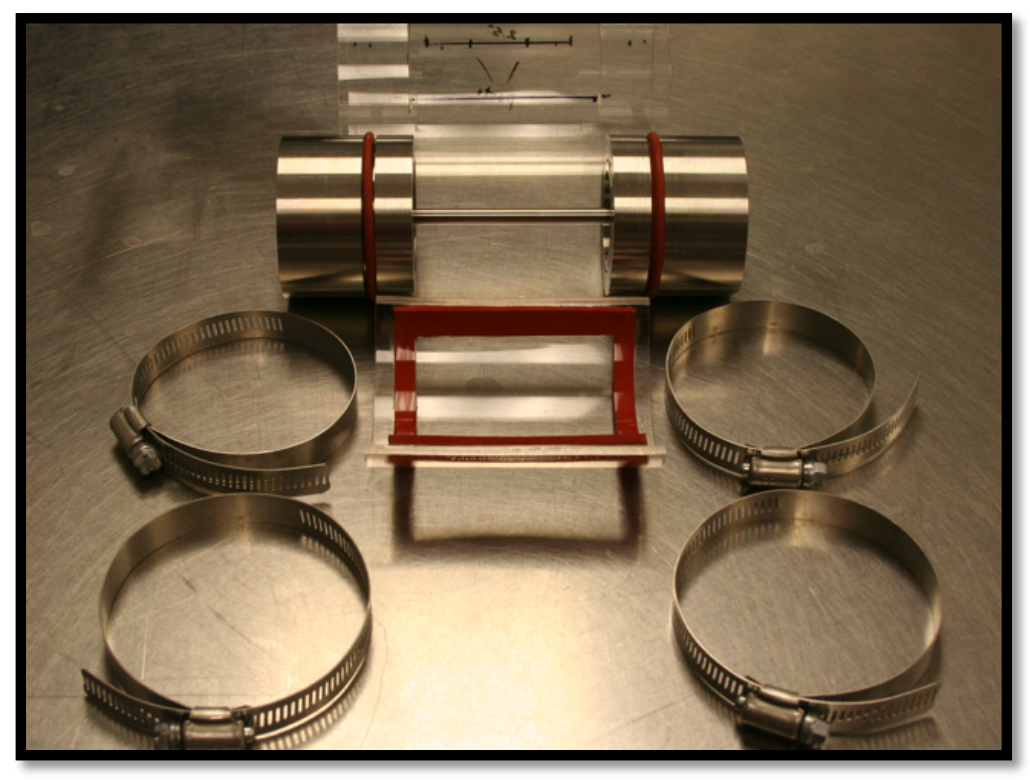

Figure 20: Initial bioreactor with scaffold cap, outer cap, outer housing, bioreactor cap, and clamps.

Phase II dealt with scaffold installation, packaging, and sterilization of the bioreactor. Phase III dealt with testing utilization in a laminar flow hood and application of cell culture methods with the bioreactor. Several iterations of design changes to the bioreactor were established through these phases of initial testing, all leading up to the final design of the bioreactor described in the next chapter. 


\subsection{Phase I}

\subsubsection{Leak Tests}

The tests under Phase I were used to evaluate the design of the bioreactor and its function when the bioreactor was internally exposed to fluid. Leak tests were the first of many water experiments used to assess the bioreactor's function. Water was chosen as it had viscosity similar to the media used in the lab, but was cheap and easy to use. The seal of the outer caps was analyzed first. As the groove for the outer caps was larger in depth then specified, use of the O-rings that had been purchased for the called out groove were first utilized. This resulted in stacking the O-ring to see if a seal could be achieved. During testing, outer caps were first fitted with O-rings using 3 or 4 in some cases. The O-ring stacking was possible with the use of O-ring picks (Fig. 21). The outer caps would then be pushed onto the ends of the acrylic outer housing and water would be filled in the extraluminal space. The bioreactor cap would then be installed and resulted in a bioreactor filled with water.

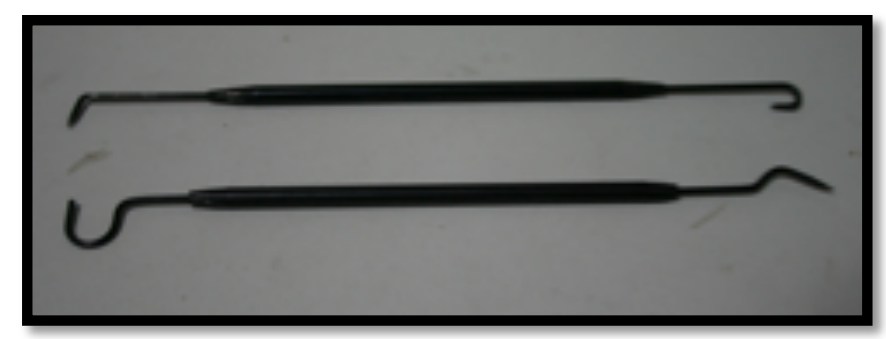

Figure 21: O-ring picks used to orientate or remove O-rings in O-ring groove.

Once filled, the bioreactor was held vertically, horizontally, and even shaken to evaluate any leaking that might occur. The bioreactor was then placed on a test tube rack, which was then placed on a paper towel. Leaks were measured if water dropped onto the paper towel marked with sharpie along the bioreactor's perimeter (Fig. 22). 


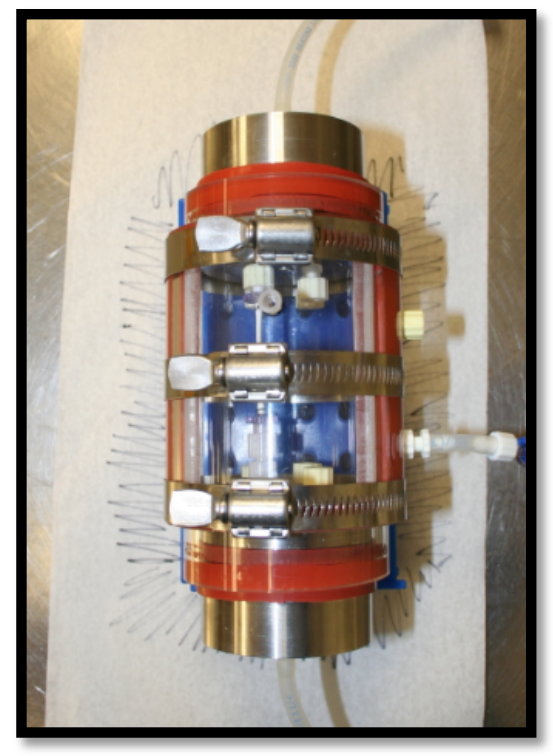

Figure 22: Bioreactor under leak test with marked perimeter to detect leaks.

With a drop of water, the ink would spread and once dried, display a distortion to the perimeter originally marked. The stacking of O-rings to create a seal worked, however one disadvantage was the difficulty of pushing the end caps into the acrylic outer housing. The difficulty originated from the high durometer specified for the O-rings. The next step was to apply O-rings with softer durometers to decrease the complexity of overall use. O-ring selection was based on material and compatibility with sterilization procedures used on campus, including EtO and autoclave. The table below shows all the O-rings that were tested to see which was the most optimal for use (Table IV).

Table IV: List of O-rings tested

\begin{tabular}{|c|c|}
\hline O-Ring & Comment \\
\hline Hard Silicone O-ring, As568 Dash Number 228 & Autoclave and EtO capable \\
\hline Hard Buna-n O-ring, As568a Dash Number 228 & $\begin{array}{c}\text { Not recommended for Autoclave or } \\
\text { EtO }\end{array}$ \\
\hline Pfte O-ring, As568 Dash Number 228 & $\begin{array}{c}\text { Stiffer than Silicone and Buna-n O- } \\
\text { rings. Produces too much friction. }\end{array}$ \\
\hline
\end{tabular}


As softer durometers were used in the outer caps, there was difficulty with the O-rings remaining in place and their ability to seal. The use of softer durometers increased ease of use of the bioreactor but decreased the ability to create a seal. The next idea was to change the design of the acrylic outer housing to keep the low durometer O-rings and maintain ease of use. This transitioned from the first to second generation acrylic outer housing.

The second-generation outer housing was designed with the same dimensions, but given a longitudinal cut .125" in width (Fig. 23).

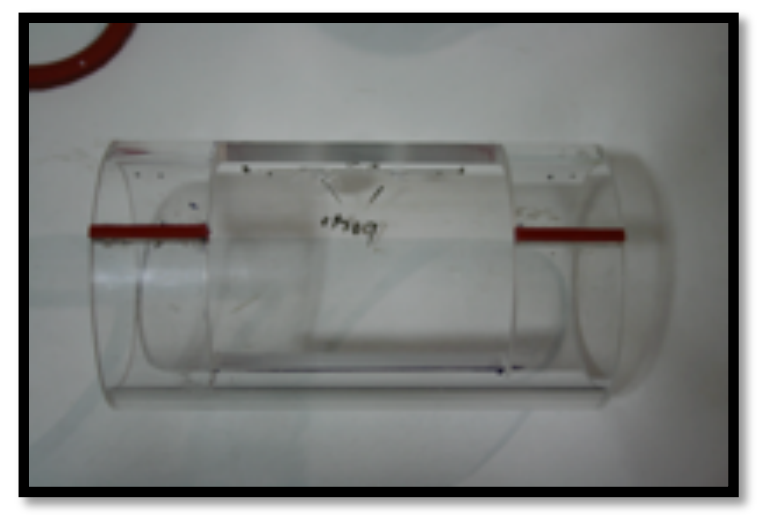

Figure 23: Second-generation housing designed with 0.125 " longitudinal cut and silicon material filling the gap.

The window located on the acrylic outer housing was larger to provide more space to reach into the bioreactor. This gap was then replaced with a piece of silicon rubber with an adhesive backing with a durometer of 50A. Stainless-steel hose clamps used to secure the bioreactor cap were used to clamp the acrylic outer housing tightly around the outer caps at the O-ring grooves. When tested, assembly was still difficult, as it required two people to pull open the outer housing and slide in the cartridge. When testing with water, leaking had occurred over several iterations of the test. As the bioreactor cartridge would 
slide into the housing, O-rings originally fixed in their grooves moved out of place affecting the seal (Fig. 24). This was assessed by repeated cartridge insertion.

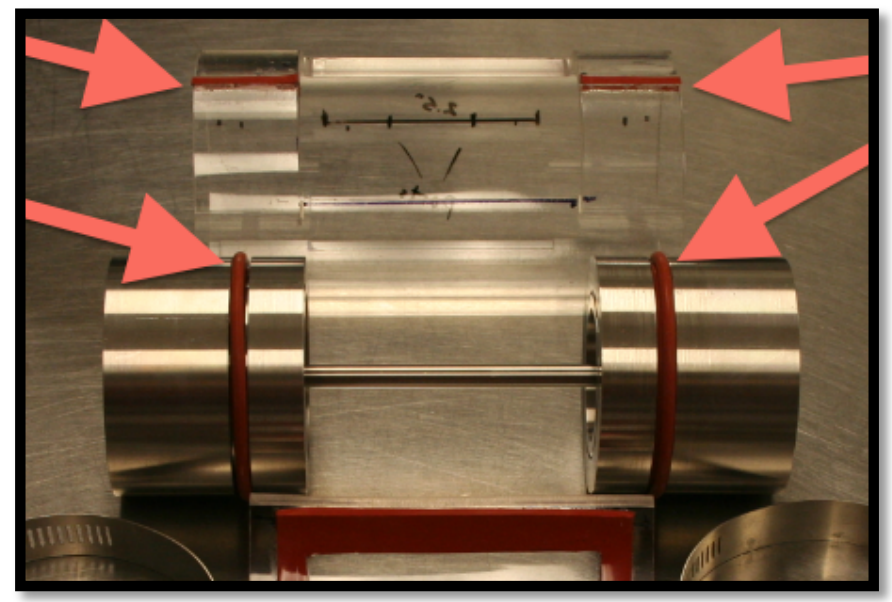

Figure 24: Second-generation bioreactor housing with cartridge (bottom) and housing (top) separated. Red arrows indicate the interfaces where leaks occurred.

In some cases, over tightening of the clamps would over compress the longitudinal gap deforming the outer housing to an ellipse where leaking would be apparent. At this stage of testing, a more reliable housing and seal were needed. Design changes went back to the first generation housing, but a focus was made to find better sealing techniques.

One solution was the manufacturing of a custom O-ring in-house to seal the bioreactor along with using the first generation acrylic outer housing. A silicon sheet of 7/16" in thickness was placed in the laser cutter provided by the Bonderson 60 machine shop. Based on the dimensions of the O-ring groove, a square O-ring was created (Fig. 25). 


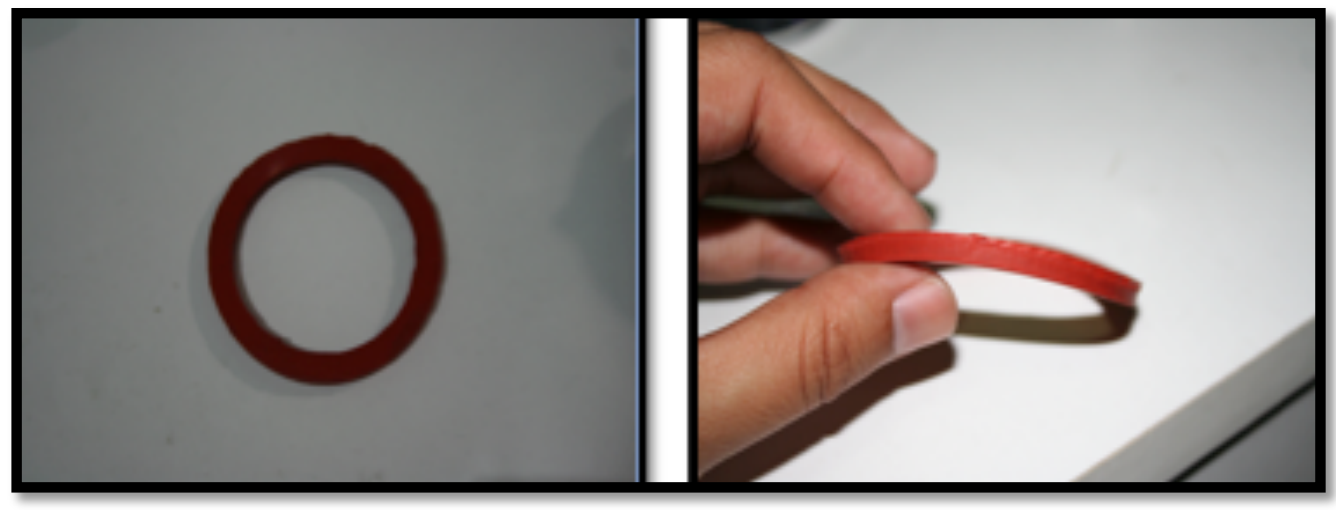

Figure 25: Custom square O-ring machined in-house using laser cutter.

Testing the seal on this design resulted in a very small leak. This was a result of a tilt that was noticed, as the component was situated in the outer housing. This resulted in leaks that would affect long-term cultivation studies.

Another solution was to utilize the area before and after the O-ring groove to place a silicon material around the outer diameter of the outer caps. The material was a silicon sheet with foam integrated within the core of the material, and an adhesive backing. Lengths were cut and dimensioned based on the perimeter of the outer diameter of the outer caps. Assembly into the outer housing was accomplished with no complications compared to previous uses of O-rings (Fig. 26).

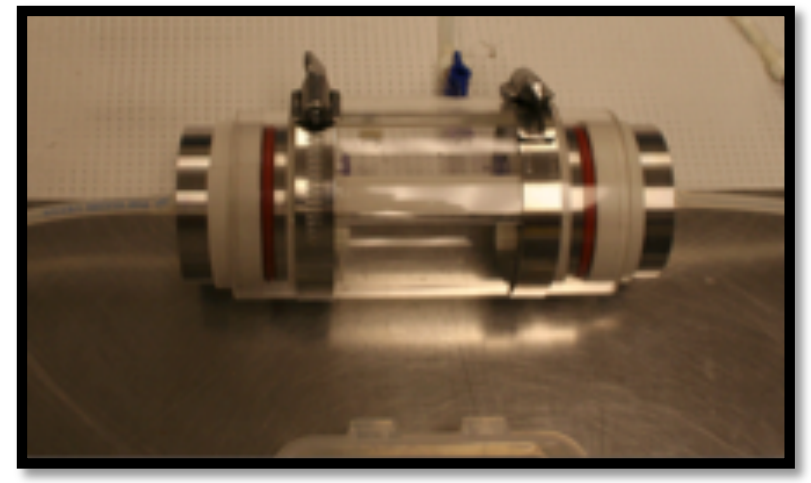

Figure 26: Assembled bioreactor with silicon foam sheet material used to seal the bioreactor. 
No signs of leaking were apparent when tested with water. This design became a potential solution to sealing the bioreactor, but complications started to arise when using this design for subsequent tests. It was discovered that the material was not able to create the robust seal during repeated tests. Every test meant replacing the material on the outer cap, which involved using acetone on the outer caps and 70\% isopropyl alcohol on the bioreactor cap to remove the residual adhesive left from the material. In terms of cost, this design would not have been efficient. In terms of upkeep of the bioreactor, this design increased maintenance time. By choosing a durometer one tier higher, a silicon material without the foam core was chosen.

The final solution was a combination of the silicon sheet material and the custom O-ring (Fig. 27). This combination increased the surface area of the seal and alleviated the tilt observed when just using the custom O-ring. The silicon sheet material was designed to be permanent on the outer diameter of the outer cap. A water test was conducted to assure that no leaks would occur under this design. The results of the water tests confirmed that this was the final iteration in the design to seal the bioreactor.

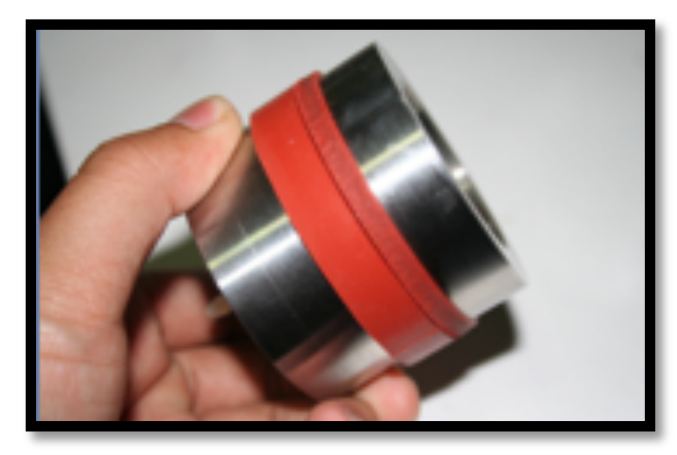

Figure 27: Final solution to sealing the bioreactor, using combination of custom square O-ring and use of silicon material along the outer diameter of the outer cap. 
To seal the contents within the outer caps from the bioreactor chamber, custom internal O-rings were placed between the scaffold cap to outer cap interface at the end of the threading (Fig. 28). To test the efficacy of the custom O-ring, water was pumped to one side of the bioreactor and the leak potential of the outer cap-scaffold cap interface was assessed. The implementation of this feature inhibited content intended for the scaffolds from escaping into the bioreactor chamber.

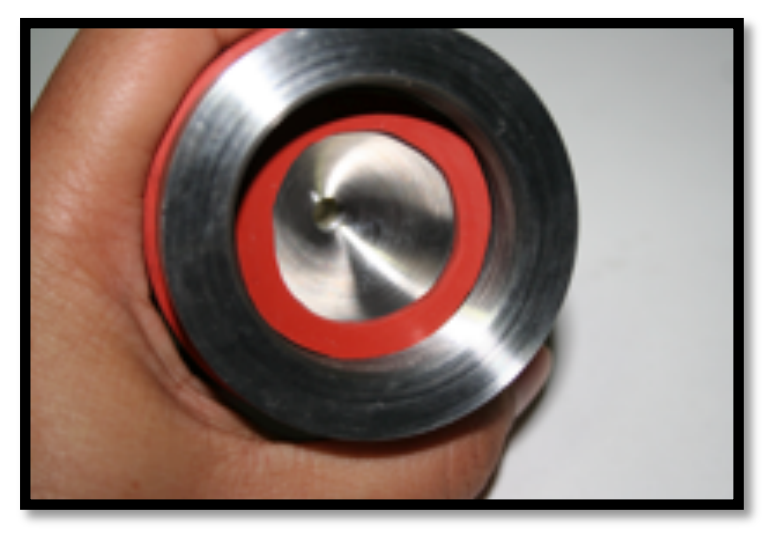

Figure 28: Custom internal O-ring used to seal inner contents of the bioreactor.

\subsubsection{Flow Characterization}

When using the bioreactor system with the perfusion pump used for the CellMax system, characterization of flow was required to know what kinds of shear stresses that would be exposing the cells to. Physiological shear stresses in the capillaries of the BBB range from 1-2 dynes $/ \mathrm{cm}^{2}[53,57,60]$. The bioreactor was assembled with $2 \mathrm{~mm}$ ID PLGA scaffolds sutured onto barbs. The tubing and chamber were filled with water and primed until all air in the system was removed. Three sets of flow rates were obtained, one for each Teflon bar including blunt end, cut end, and low flow rate pin (Fig. 29,30). Flow rates in milliliters per minute were measured at each of the eleven settings provided by the CellMax pump (Table V). Pipettes were used to measure the fluid that would exit the 
system in one minute. Using the theoretical definition of laminar flow shear stress, flow rate of milliliter per minute was converted to dynes $/ \mathrm{cm}^{2}$ (Eq. 1) (Fig. 31). Values obtained show similar values compared to flow rate and shear stress characterization of the CellMax system conducted by Bryan Brandon (Table VI).

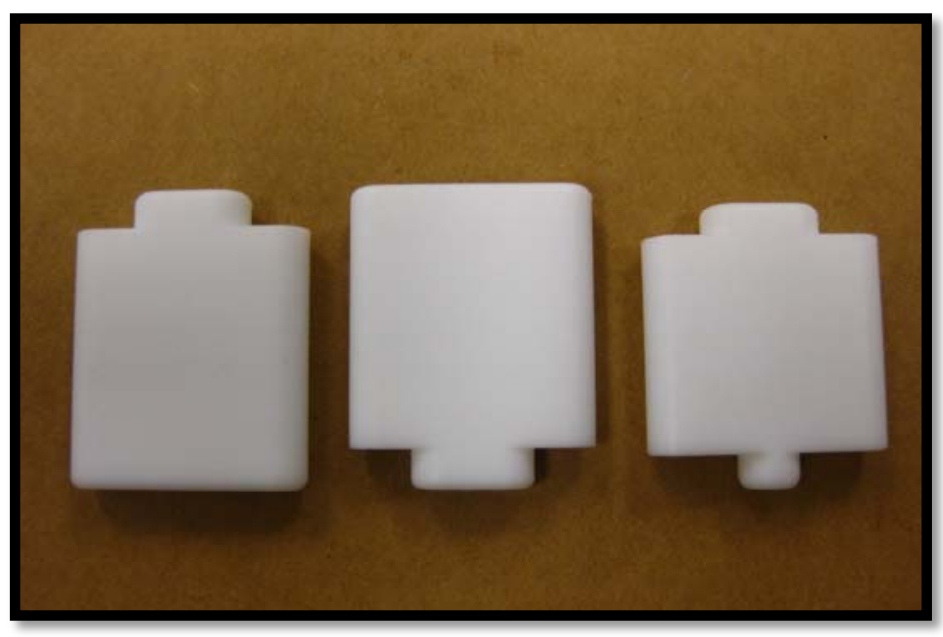

Figure 29: Three Teflon bars that were included in the CellMax pump system. The bar on the left, the blunt end pin, is capable in creating the highest flow rates. Bar in the middle, cut end pin can achieve lower flow rates. Lastly, the low flow rate pin achieves the lowest flow rates.

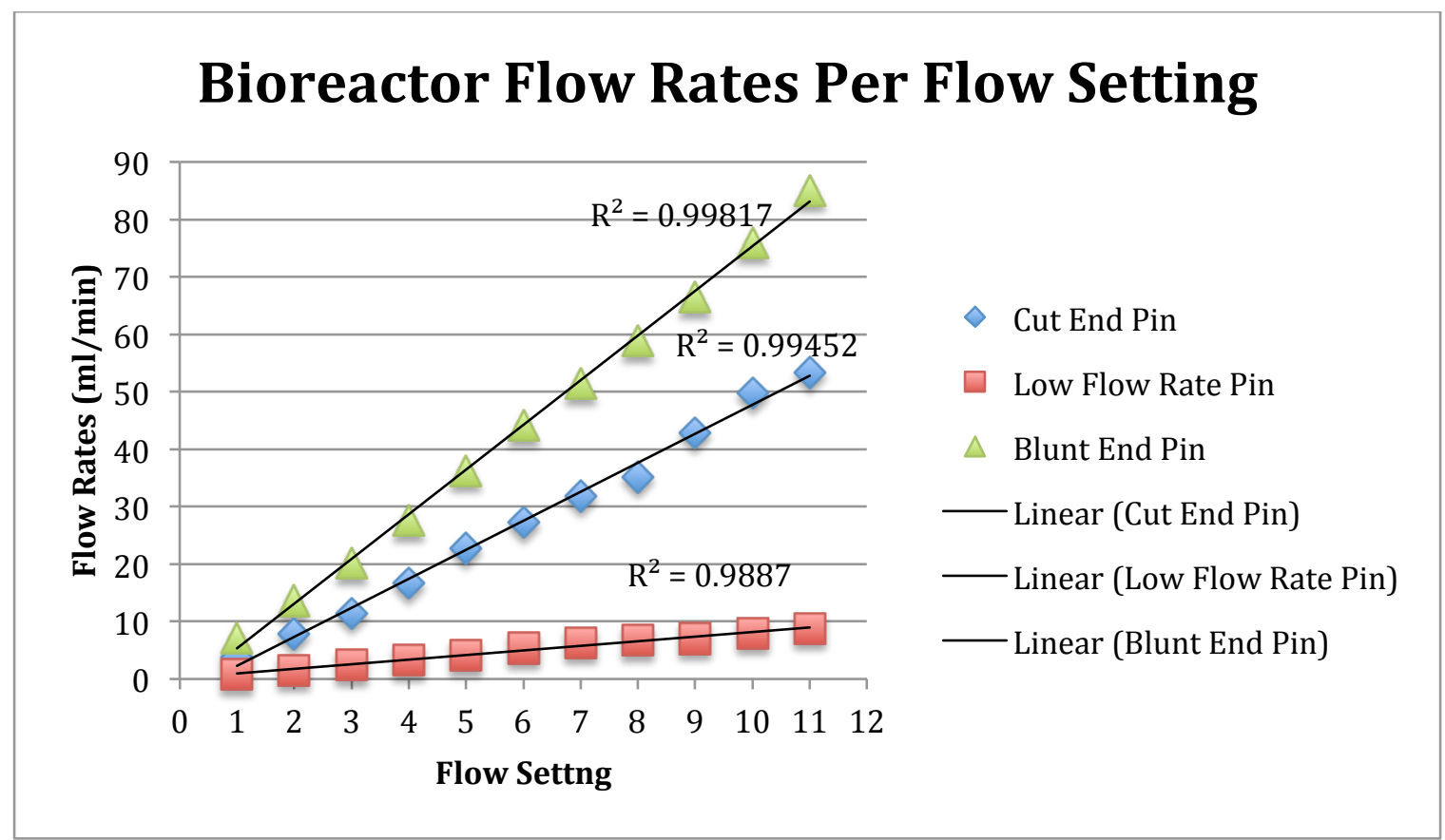

Figure 30: Flow rates in $\mathrm{ml} / \mathrm{min}$ referring to the rate experienced in bioreactor system with four scaffolds. 
Equation 1: $\quad \tau=(4 \eta \mathrm{Q}) /(\pi \mathrm{r} 3)$

where: $\eta=$ viscosity $(($ dyne sec $) / \mathrm{cm} 2)$

$\mathrm{Q}=$ fluid flow rate $(\mathrm{cm} 3 / \mathrm{sec})$

$\mathrm{r}=$ internal radius of hollow fiber $(\mathrm{cm})$

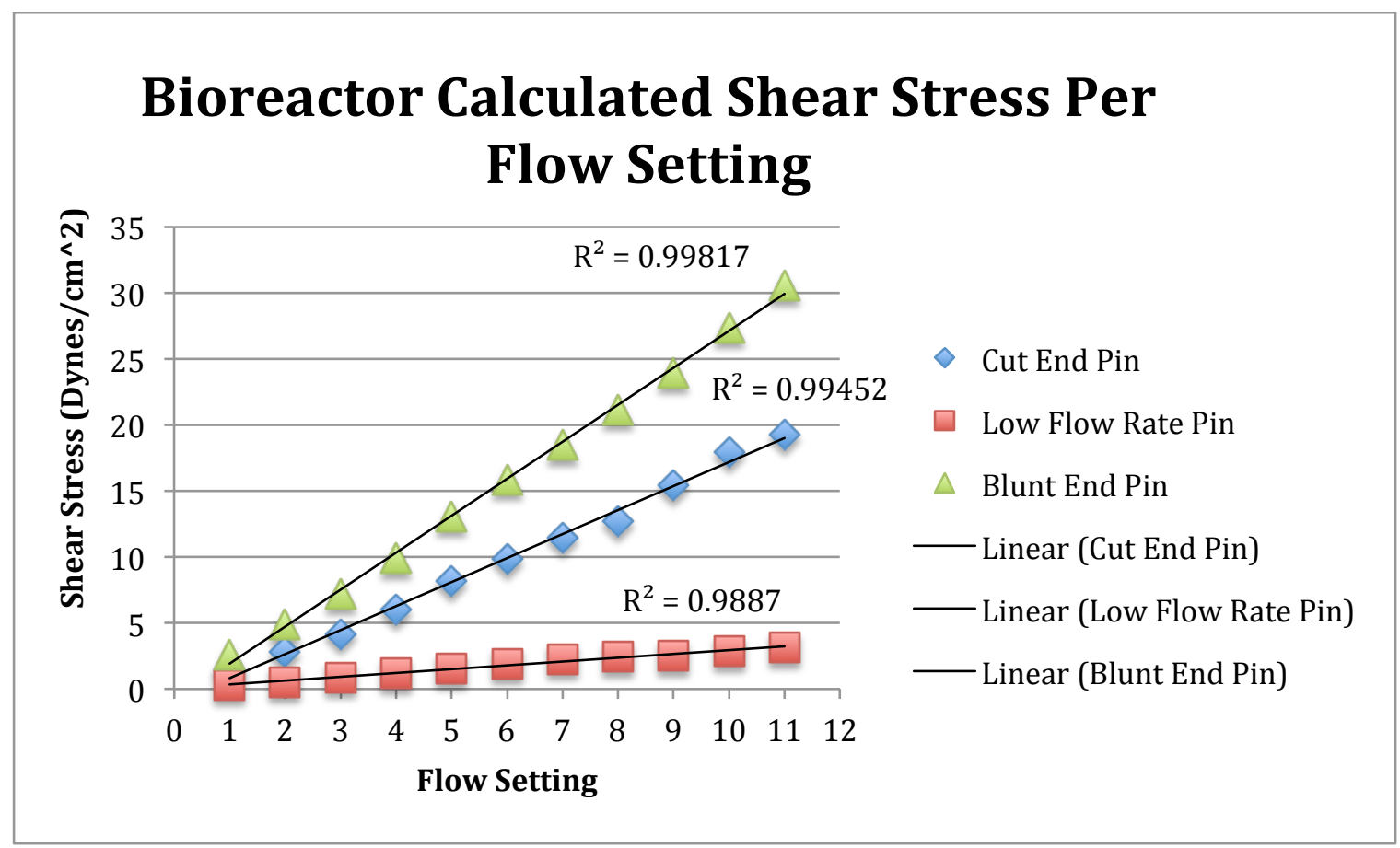

Figure 31: Each rate in dynes $/ \mathrm{cm}^{2}$ refers to the rate experienced in each scaffold in the Final BBB bioreactor. 
Table V: Table displaying flow rates in $\mathrm{ml} / \mathrm{min}$ and shear stress in dynes $/ \mathrm{cm}^{2}$ of the final bioreactor. These rates were obtained when the system was fitted with four scaffolds.

Each rate in $\mathrm{ml} / \mathrm{min}$ refers to the whole system with four scaffolds. Each rate in dynes $/ \mathrm{cm}^{2}$ refers to the rate experienced in each scaffold.

\begin{tabular}{|l|l|l|l|l|l|l|}
\hline & \multicolumn{2}{|l|}{ Blunt End Pin } & \multicolumn{2}{l|}{ Cut End Pin } & \multicolumn{2}{l|}{ Low Flow Rate Pin } \\
\hline Settings & $\mathrm{ml} / \mathrm{min}$ & dynes $/ \mathrm{cm}^{2}$ & $\mathrm{ml} / \mathrm{min}$ & dynes $/ \mathrm{cm}^{2}$ & $\mathrm{ml} / \mathrm{min}$ & dynes $/ \mathrm{cm}^{2}$ \\
\hline 1 & 7.2 & 2.60 & 3.9 & 1.4 & 0.89 & 0.32 \\
\hline 2 & 13.6 & 4.90 & 7.8 & 2.81 & 1.5 & 0.54 \\
\hline 3 & 20.1 & 7.24 & 11.5 & 4.14 & 2.5 & 0.90 \\
\hline 4 & 27.5 & 9.90 & 16.7 & 6.01 & 3.3 & 1.19 \\
\hline 5 & 36.3 & 13.10 & 22.7 & 8.17 & 4.2 & 1.51 \\
\hline 6 & 44.1 & 15.88 & 27.3 & 9.83 & 5.4 & 1.94 \\
\hline 7 & 51.4 & 18.51 & 31.8 & 11.45 & 6.3 & 2.27 \\
\hline 8 & 58.8 & 21.18 & 35.2 & 12.68 & 6.8 & 2.45 \\
\hline 9 & 66.5 & 23.95 & 42.8 & 15.41 & 7 & 2.52 \\
\hline 10 & 76 & 27.37 & 49.8 & 17.93 & 8 & 2.88 \\
\hline 11 & 85 & 30.61 & 53.4 & 19.23 & 8.79 & 3.17 \\
\hline
\end{tabular}

Table VI: Flow rate characterization performed by Bryan Brandon. Each rate in $\mathrm{ml} / \mathrm{min}$ and dynes $/ \mathrm{cm}^{2}$ refers to the CellMax system [60]

\begin{tabular}{|l|l|l|l|l|l|l|}
\hline & \multicolumn{2}{l|}{ Blunt End Pin } & \multicolumn{2}{l|}{ Cut End Pin } & Low Flow Rate Pin \\
\hline Settings & $\mathrm{ml} / \mathrm{min}$ & dynes $/ \mathrm{cm}^{2}$ & $\mathrm{ml} / \mathrm{min}$ & dynes $/ \mathrm{cm}^{2}$ & $\mathrm{ml} / \mathrm{min}$ & dynes $/ \mathrm{cm}^{2}$ \\
\hline 1 & 9 & 6.8 & 4.5 & 3.4 & 0.8 & 0.61 \\
\hline 2 & 16 & 12.1 & 8.5 & 6.4 & 2 & 1.52 \\
\hline 3 & 23 & 17.4 & 13 & 9.8 & 2.75 & 2.08 \\
\hline 4 & 30 & 22.7 & 17.5 & 13.3 & 3.75 & 2.84 \\
\hline 5 & 35 & 26.5 & 21 & 15.9 & 4.25 & 3.22 \\
\hline 6 & 42 & 31.8 & 26 & 19.7 & 5 & 3.79 \\
\hline 7 & 50 & 37.9 & 31 & 23.5 & 5.5 & 4.17 \\
\hline 8 & 57 & 43.2 & 36 & 27.3 & 6.25 & 4.73 \\
\hline 9 & 67 & 50.8 & 39 & 29.5 & 7 & 5.3 \\
\hline 10 & 71 & 53.8 & 43 & 32.6 & 7.5 & 5.68 \\
\hline 11 & 80 & 60.6 & 48 & 36.4 & 7.25 & 5.49 \\
\hline
\end{tabular}




\subsection{Phase II}

Scaffold installation was characterized by ease of installation, time spent in the hood, and the risk of contamination. Installing the scaffolds before or after sterilization in a laminar flow hood was evaluated. Installation while in the hood was the more difficult of the two. This method required the use of sterile gloves so that the user had the ability to pass his or her hands over objects. Sterile sutures were needed to secure the scaffold onto the barbs. This procedure was also the most time consuming, taking close to 20 minutes per scaffold. In addition, this led to increases in window dimensions to account for more space for users to enter the bioreactor chamber. This method was challenged by installation of scaffolds prior to sterilization. This procedure of installation reduced the risk of contamination and decreased time spent during experimental set up. The installation of scaffolds prior to sterilization was determined to be the more efficient method based on time of installation and difficulty for the user.

\subsubsection{Packaging \& Sterilization}

Packaging of the bioreactor was another phase of testing to see if the bioreactor could comply with techniques already used in the lab. Packaging of the Tupperware bioreactor involved the use of Paper/Film Gas/Steam Sterilization Pouches supplied by Cardinal Health. The dimensions for the large blue film self-seal pouches were $7.5 \times 13$ ", fitting the Tupperware bioreactors quite well. However, the bioreactor system was too large to fit into one pouch, so the system was divided into three bags (Fig. 32). 


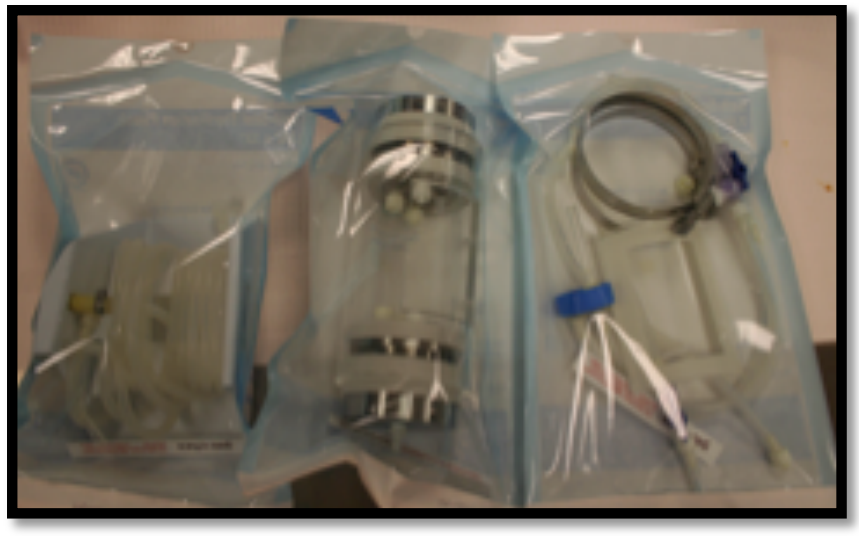

Figure 32: Bioreactor packed in three 7.5" x 13" sterilization pouches.

To increase efficiency and to decrease time spent in the hood, larger pouches were purchased so that the whole system could be fully assembled, sterilized, and opened in one pack. The larger packs had dimensions of $13 \times 18$ ", and were able to fit the bioreactor and pump tubing (Fig. 33).

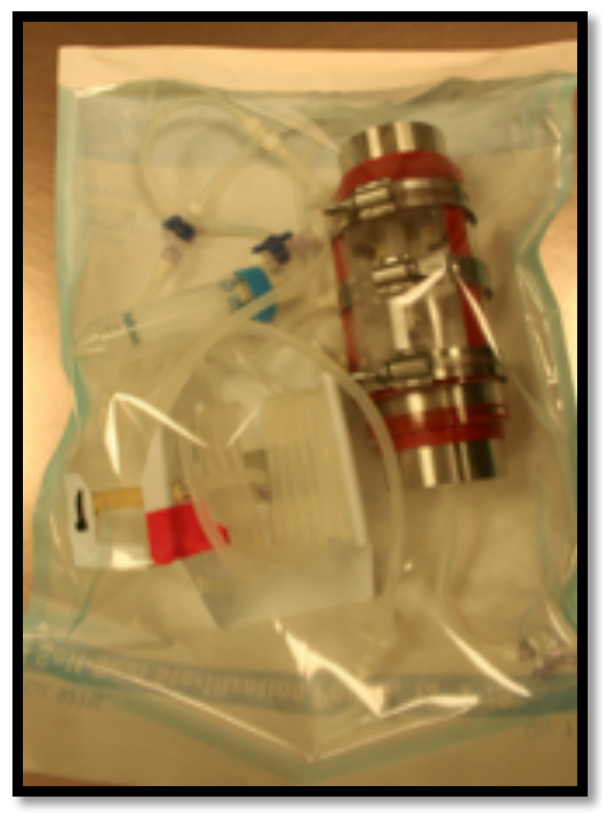

Figure 33: Bioreactor system packaged in one 13" x 18" sterilization pouch. 
Sterilization was the other half of phase II testing, exploring methods available on campus and in the Tissue Engineering Lab. The Veterinary Clinic on campus provided an Ethylene Oxide sterilizer, while an autoclave was available in the Biomedical Engineering Department. Both methods were investigated to assess which was best, starting with the autoclave since it was easily accessible.

The specifications of the autoclave included a temperature range of $250-300^{\circ} \mathrm{F}$. All main components of the bioreactor including outer housing, outer caps, scaffold caps/bar, internal outer cap O-ring, and internal fitting had melting temperatures above the temperature specifications of the autoclave. Before placing the bioreactor inside the autoclave, samples from each of the main components of the bioreactor were placed in the sterilizer and processed under the autoclave setting "Packs". Measurements of dimensions were taken for each sample before and after sterilization to assess deformation. Once each sample was cleared to withstand autoclave sterilization, testing the bioreactor fully assembled was next to be done. Once packaged, the bioreactor was set to undergo the "Packs" setting for the duration of one hour (Fig. 34).

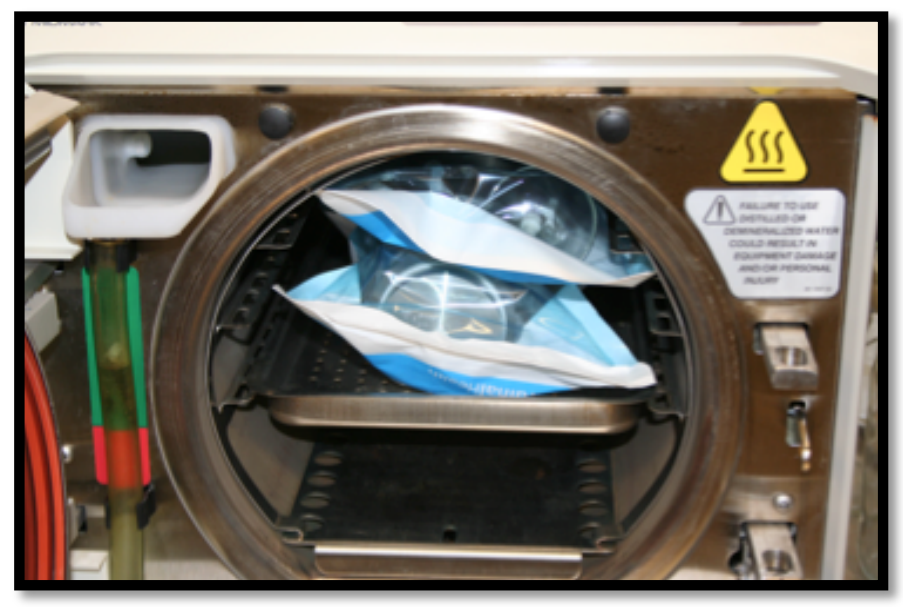

Figure 34: Bioreactor packages placed into autoclave for sterilization. 
When sterilization finished, the package needed time to cool off, as the stainlesssteel parts were still hot. Damage was first seen when the acrylic outer housing had warped and deformed near the interface of the outer caps. This was first seen to be a problem, but additional leak tests proved that the sterilization processes did not ruin the seal of the bioreactor. The second observation was that the stopcock fittings were loose and could no longer engage in their locking positions. This became problematic as the autoclave shrunk internal parts of the stopcock. This showed that with the bioreactor fully assembled, autoclave was not a feasible method for sterilizing.

EtO sterilization was then assessed, posing as an alternative to autoclave sterilization. The tissue-engineering lab used the EtO sterilizer at the Vet Clinic to sterilize bioreactors intended for sterile set-ups. The lab was charged per lot for the ethylene gas used, and services were only available on the weekends. The specifications for EtO include a temperature range of $100-140^{\circ} \mathrm{F}$ and very minimal pressures. The sterilization differs from autoclave, as it does not use steam to kill microorganisms, but instead uses gas to sterilize. The one disadvantage of using EtO was the residue that could have resided on the surfaces or pores of materials. This was a potential challenge for pre-loading PLGA scaffolds into the bioreactor prior to sterilization as there was concern that the residue could be trapped within the pores of the scaffold, and would cause a cytotoxic effect on the cells. Sterilizing with EtO meant that the bioreactor system was fully assembled and left as a semi-closed system. Semi-closed refers to having one of the transmural ports open to allow gas to enter the bioreactor chamber and sterilize scaffolds. The bioreactor cap port was also open to allow gas entry into the chamber as well as a way for exiting gas and pressure. At the end of the sterilization 
cycle, the process did not damage the bioreactor. EtO therefore became the main sterilization process for the bioreactor, but later investigation of cell viability was required to assess whether the scaffolds could be preloaded, and if the residue was killing the cells [67].

\subsection{Phase III}

Phase III of initial testing consisted of testing the bioreactor in the hood and applying cell culture methods. Before testing inside the hood, a grid was created to mimic the hood dimensions on a bench top, in order to determine how the packaged bioreactor would enter, be placed, and opened in the hood. Orienting the packaged bioreactor to the upper right area of the hood left the middle area for loading of bioreactor media, as well as placing end caps to open ports. Assessing how to insert bioreactor media into the chamber involved strategies to reduce risk of contamination (Fig. 35).

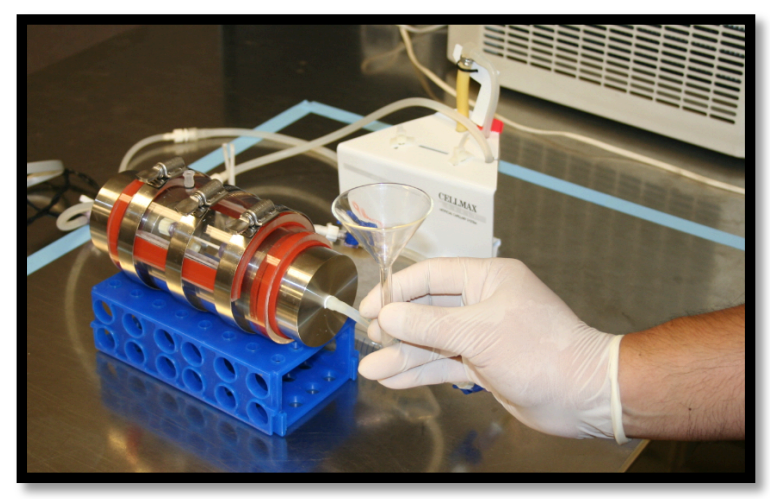

Figure 35: Funnel technique used to insert bioreactor media into bioreactor chamber.

The first technique was to open the hose clamps and remove the bioreactor cap to allow entry into the chamber. It was then discovered during filling of the bioreactor chamber, that as window size increased, space to hold the fluid in the chamber decreased. This risked the users ability to fully submerge the scaffolds with bioreactor media. During 
single or double scaffold set-ups, this would not be a problem, as the two scaffolds would be situated on the lower level. With the time of installing the scaffolds and the addition of bioreactor media into the chamber, this method took an hour in the hood. To address time constraints and risk of contamination when opening the bioreactor, a funnel technique was used. This funnel technique included a glass funnel and the transmural outlet tubing. Pouring of the bioreactor media into the chamber was accomplished without ever opening it. This allowed the media level to go past the outer diameter of the outer caps, and fully submerge four scaffolds (Fig. 36).

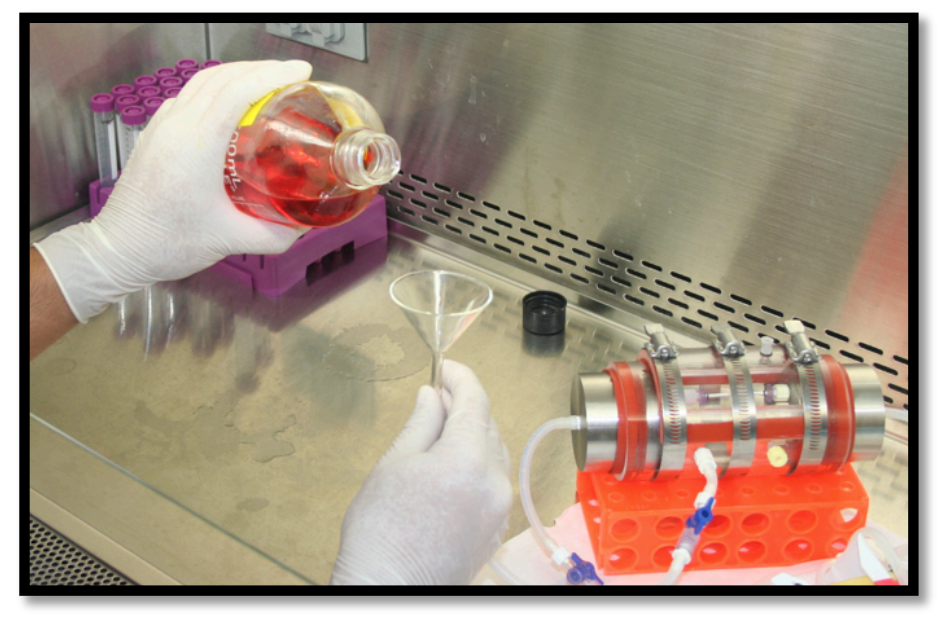

Figure 36: Process to fully submerge scaffold barbs.

Compared to the non-funnel method, the funnel approach took 15 minutes and decreased the amount of difficulty using the bioreactor. Because of this, the funnel method was chosen to be in the final protocol for use. This also lead to the design change of the bioreactor cap to be fitted with a transmural port, to relieve pressure as the bioreactor chamber was being filled. Based on students using the bioreactor to gain feedback in accessibility, it was determined that the stabilization bar hindered navigation inside the chamber during scaffold installation. After assessment that more room could be 
obtained in the absence of the bar, the stabilization bar was removed and replaced with a plug. The outcome of all the initial phase testing resulted in the final design of the bioreactor with protocols for overall use and maintenance, as described in the following sections. 


\section{Chapter 4: Final Design of BBB Bioreactor}

\subsection{Final Design}

This chapter describes the final components and assembly of the bioreactor system. The custom, in-house bioreactor system was required to meet the following design constraints: sterilizability, reusability, easy of use, leak-free, and culture up to four scaffolds at once. The results of the initial phase testing described in the previous section, as well as the associated design changes led to the final design of the bioreactor described in this chapter (Fig. 37). Finalized protocols were also created including protocols for assembly, conditioning, cell culture, take down, and cleanup (Appendix D).

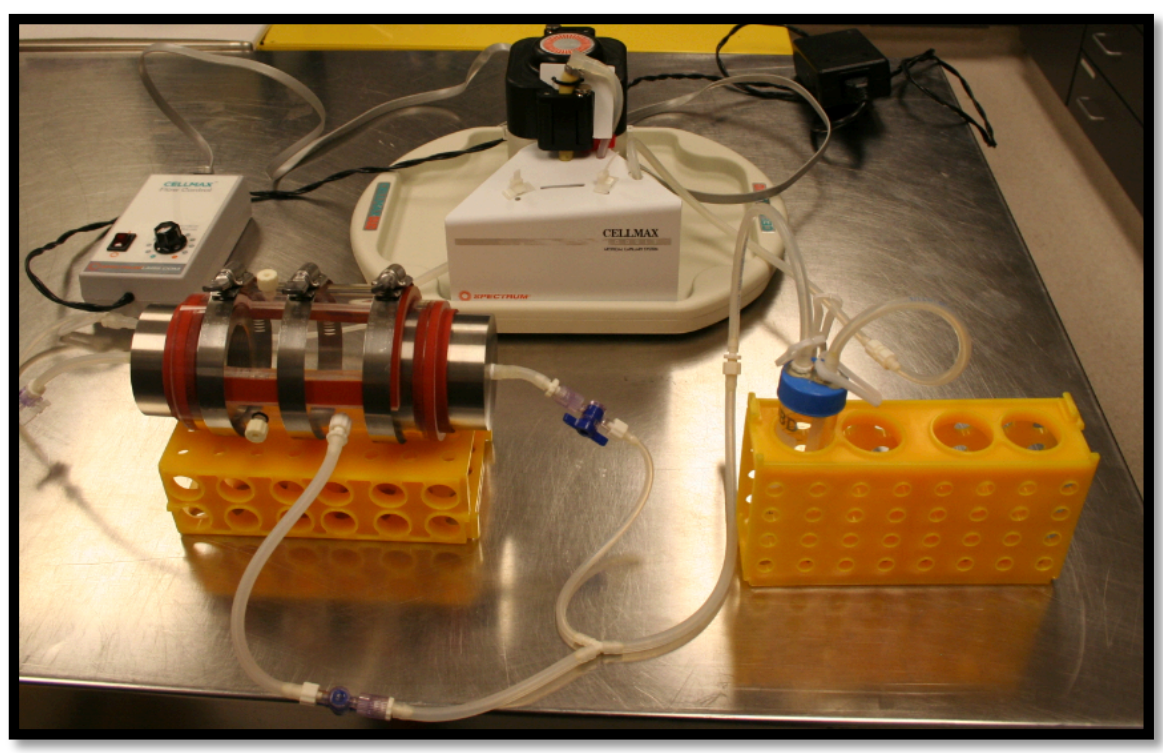

Figure 37: Final design of bioreactor system with pump and media reservoir.

\subsection{Components}

This final version of the bioreactor improves upon the components seen in previous designs. In this version, the stabilization bar was removed to provide more space for scaffold installation. These scaffold caps were fitted with \#10-32 holes for both male and female luer-to-thread fittings. To prevent leaks the custom square O-ring and the 
silicone strip material was mounted on the outer diameter of the outer caps. Two transmural ports were located on the side of the bioreactor to enable sampling, filling, and allow for transmural flow as shown in Figure 38. Stainless-steel hose clamps were used to secure the bioreactor cap providing a seal. The transluminal port located on the cap is only accessed during introduction of bioreactor media to relieve pressure build up. Finally, a 50ml conical was included in the tubing to serve as a media reservoir.

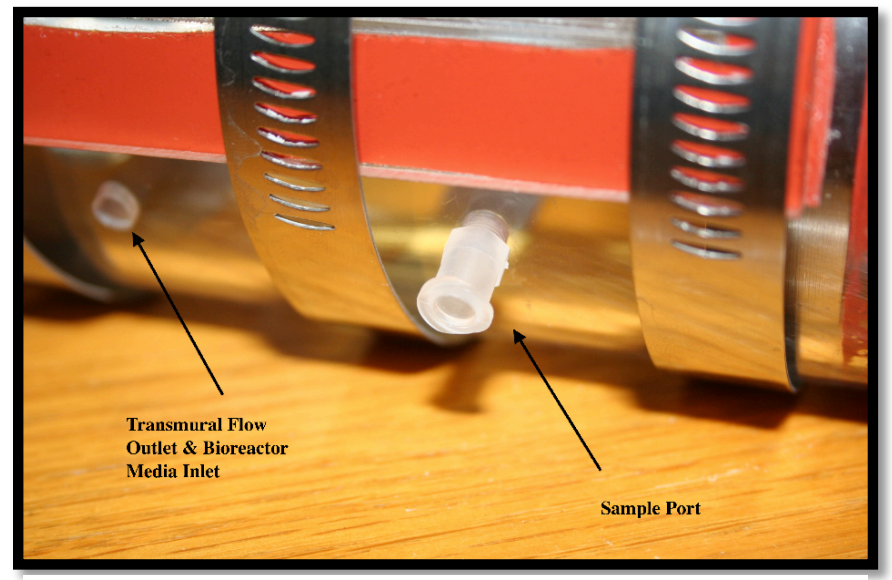

Figure 38:Transmural outlets located on the side of the bioreactor.

\subsection{Assembly}

Constructing the bioreactor was a four-part process including scaffold installation, outer cap insertion, bioreactor cap placement, and connecting the appropriate tubing. A detailed step-by-step protocol can be found in Appendix D, and is summarized below. Assembly, which took approximately twenty minutes, was designed so that the whole system was a semi-closed system before EtO sterilization. 


\subsubsection{Scaffold Cap Preparation}

With the scaffold caps facing up, four male luer-to-thread fittings and four female luer-to-thread fittings were threaded onto the caps (Fig. 39). Custom O-rings were then placed into the outer caps to provide a seal between the inside of the outer cap and the bioreactor chamber. Caps were threaded into the stainless-steel outer caps, and oriented so that all eight barbs were aligned with each other.

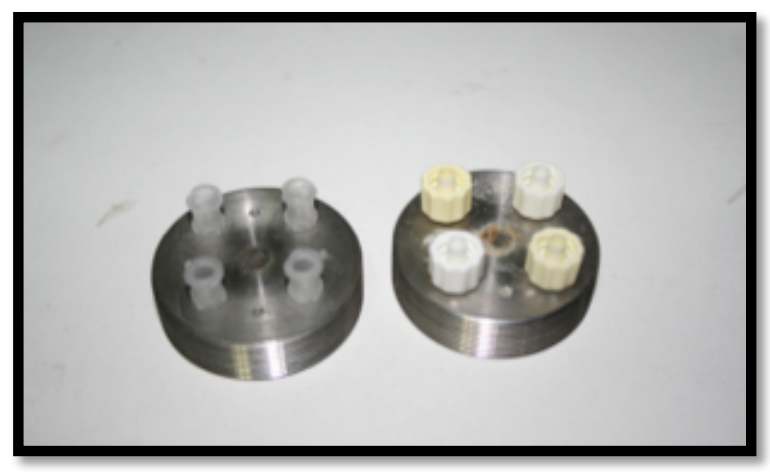

Figure 39: Scaffold caps installed with their associated fittings.

Once the scaffold cap and outer cap were now connected, scaffolds could be fitted onto one of the outer caps. Using the Qosina barbs and silk threaded sutures, the PLGA scaffolds were sutured tightly around the barbs (Fig. 40). Once secured, one end of the barb-scaffold-barb component was connected to the male luer-to-thread fittings and the other end to the male-to-male luer lock connectors (Fig. 41). 


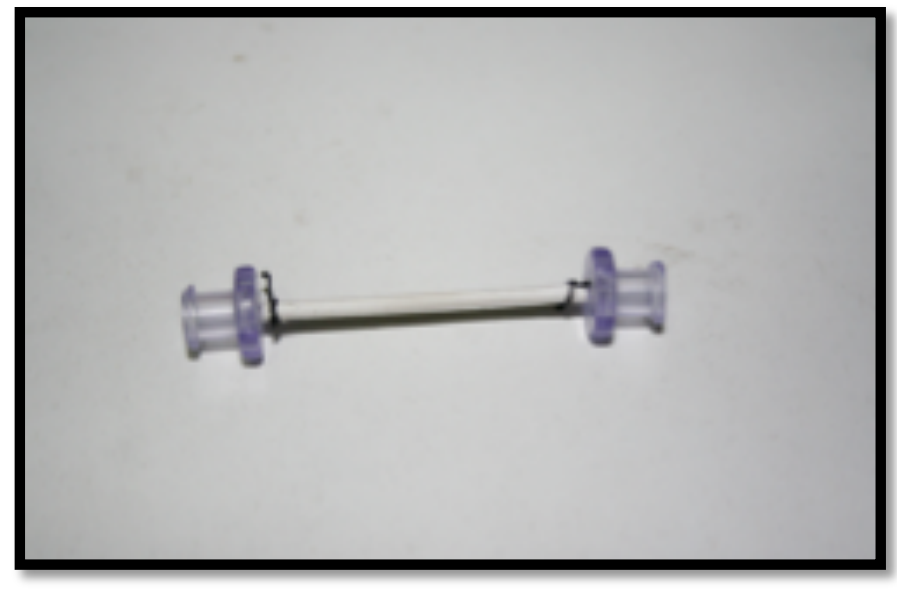

Figure 40: Scaffold sutured to two Qosina barbs ready to be installed into bioreactor.

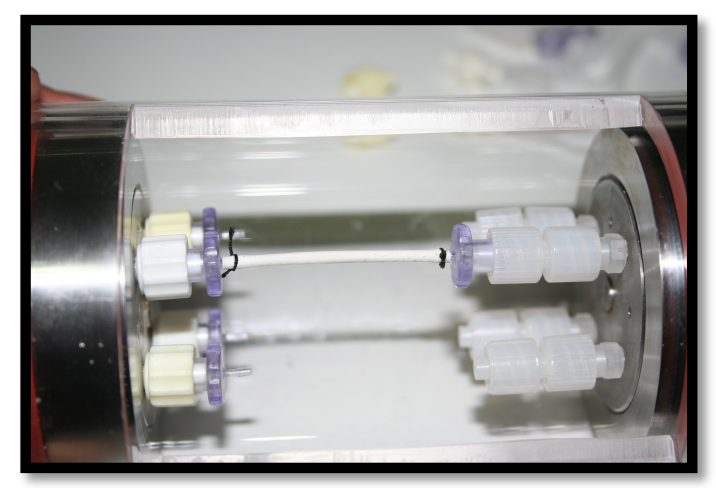

Figure 41: Scaffold installation into bioreactor.

\subsubsection{Outer Cap Installation}

After scaffold installation, the two outer caps were then ready to be inserted into the acrylic outer housing to construct the bioreactor. The outer caps were pushed into the outer housing until there was $1 / 8$ " of silicone still visible from the edges of the housing. Simultaneously, alignment of the end of the barbs and the male-to-male fittings was important so the scaffolds could be connected. Once aligned, scaffold barbs were secured by turning the male-to-male fittings counterclockwise, securing the luer lock connection. 


\subsubsection{Bioreactor Cap}

The bioreactor cap was then placed over the outer housing window. The cap was centered over the window, as alignment of the cap was important to maintain a seal of the chamber. Once placed, the window was pressed firmly to assure that all surfaces of the silicone were touching the acrylic housing. Three stainless-steel hose clamps were then placed around the housing and cap to close the bioreactor and prevent leaking. Following this assembly, the rest of the tubing was connected to complete the system described below.

\subsubsection{Tubing \& Media Reservoir}

Once the bioreactor was assembled, tubing was connected to provide flow from the media reservoir, through the pump tubing, into the inlet of the bioreactor, through the outlet (transmurally or transluminally), and back into the media reservoir. Starting with the outlet of the media reservoir, tubing was connected the pump and then the inlet of the bioreactor. The outlet tubing of the bioreactor was then connected with the inlet tubing of the media reservoir, completing a cycle back to the outlet of the reservoir (Fig. 42). 


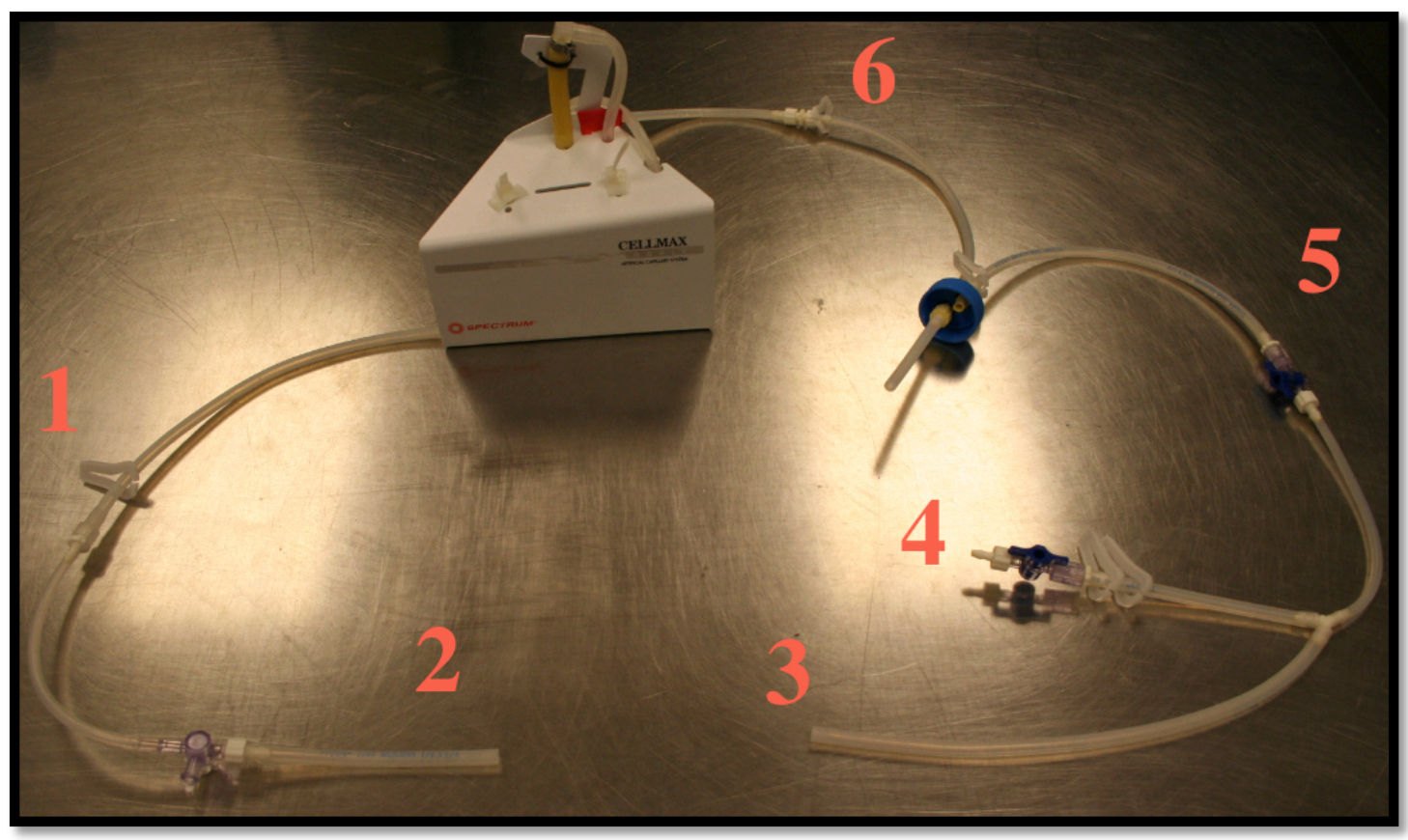

Figure 42: Inlet Tubing: Connect one end of the inlet tubing to the outlet connection of the pump tubing (1). Then, connect the other end to the inlet of the bioreactor (2).

Outlet Tubing: There are three ends in the outlet tubing. Connect one end to the outlet of the bioreactor (3), and another end should be connected to one of the transmural ports on the side of the bioreactor (4). The last end should be connected to the outlet port of the media reservoir (5).

Pump Tubing: Connect the inlet connection of the pump tubing to the inlet port of the media reservoir (6). The outlet connection should be connected to the inlet tubing of the bioreactor.

Assembly protocols for the tubing and media reservoir as well as the previous assembly processes mentioned above were further described in detail in Appendix D. As the assembly protocols were finalized, the system was ready to perform cellular testing to further evaluate the capabilities of the bioreactor. 


\section{Chapter 5: Cell Testing Methods, Results, \& Discussion}

\subsection{Introduction}

Once the assembly process had been finalized and documented, the next step was to test the system with cells. Testing with cellular components progressed from nonsterile tests, to sterile tests, and from single scaffold tests, to four scaffold tests. Each test further evaluated the capabilities and performance of the bioreactor, with a focus on aspects such as leaks, cell distribution, sterility, cell viability, and long-term cultivation. Single scaffold studies were presented as proof-of-concept studies and four-scaffold tests were designed to evaluate cell densities and cell distribution in proximal, middle, and distal regions of the scaffolds. "Proximal" refers to the region being closest to the inlet of the bioreactor, while "distal" refers to a region farthest away from the inlet. "Middle" was the region in between the two. Multiple-scaffold tests also investigated flow distribution between top, bottom, left, and right scaffold ports. Fibroblasts were chosen as the cell type for all tests due to the ease of culture, maintenance, and lower cost compared to other cell types used in the lab. Methods for sterile or non-sterile, single scaffold, and multiple scaffold tests are outlined below. Detailed protocols of all tests can be referred to in Appendix D.

\subsection{Sterility Evaluation}

Contamination can be a result of microorganisms entering the bioreactor system.

During cell culture, contamination typically does not become a problem until after the first twenty-four hours. Therefore, non-sterile studies were conducted in twenty-four hours or less. In order for the bioreactor to perform long-term culture, sterile conditions were necessary to recreate normal culture conditions such as $\mathrm{pH}$. Bioreactor tests were 
conducted using non-sterile for short-term studies or sterile techniques for longer-term studies. Non-sterile conditions presented information quickly, allowing quick assessment of the presence and the distribution of the cells on the scaffold. Assembly of the bioreactor was done outside of the laminar flow hood. After the bioreactor was then brought into the hood, bioreactor media was poured into the chamber, and conditioning media was poured into the media reservoir. The bioreactor pump tubing was then brought into the large incubator, where the pump would be installed. Pump installation involved securing the pump tubing into the CellMax pumping station. The bioreactor was then primed to allow all air to be expelled from the system (Fig. 43).

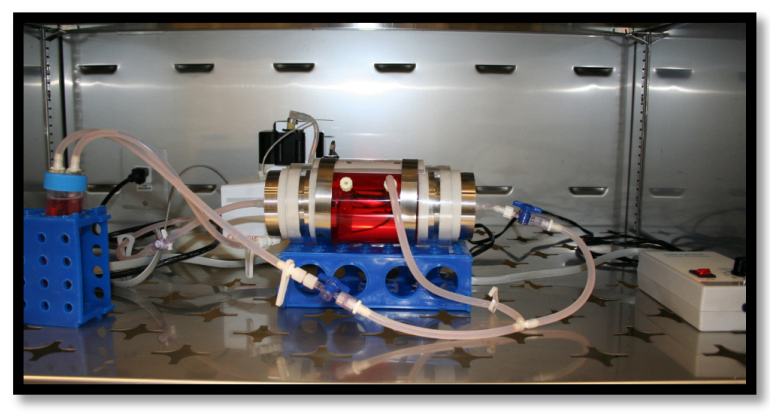

Figure 43: System primed before transmural flow.

Once the system was primed, the bioreactor was ready for flow. Sterile experiments involved more restrictions in terms of handling compared to non-sterile setups. Bioreactor handling occurred inside the hood with the use of sterile gloves. Results are summarized in the table below indicating which protocols maintained sterility. Sterile protocols resulted in the most successful experiments across all types of testing. After standardizing protocols for using the bioreactor, single-scaffold evaluation was next to assess the presence of cells and cell distribution on a scaffold. 
Table VII: Evaluation of Sterile and Non-sterile protocols across all types of tests

\begin{tabular}{|l|l|l|l|}
\hline & $\begin{array}{l}\text { Problems in 24 hour } \\
\text { single-scaffold set- } \\
\text { up }\end{array}$ & $\begin{array}{l}\text { Problems in 7-day } \\
\text { single-scaffold set- } \\
\text { up }\end{array}$ & $\begin{array}{l}\text { Problems in 7-day } \\
\text { multi-scaffold set- } \\
\text { up. }\end{array}$ \\
\hline Non-Sterile Protocol & Yes & N/A & N/A \\
\hline Sterile Protocol & No & No & No \\
\hline
\end{tabular}

\subsection{Single-Scaffold Testing Methods \& Results}

Single-scaffold testing allowed the assessment of the presence and distribution of cells and the ability of long-term culture of up to seven days. Both sterile and non-sterile experiments were conducted for single-scaffold tests. The first single single-scaffold test was a non-sterile twenty-four hour set-up. A twenty-four hour conditioning period was implemented to allow proteins in the media to radially stick onto the inner lumen of the scaffold prior to sodding of cells. The experiment involved one 100\% confluent T75 flask of 3T3 fibroblast cells, providing around 3-4 million cells to the bioreactor. Pump settings maintained at a rate of $1 \mathrm{ml} / \mathrm{min}$ or a shear stress of $1.4 \mathrm{dynes} / \mathrm{cm}^{2}$ [62]. At the end of the culture period, scaffolds were extracted and evaluated for cell presence and distributions using a Bisbenzimide (BBI) stain. Images were taken at 40X magnification at proximal, middle, and distal regions. Sterile single-scaffold testing followed the same protocol described in section 5.2 to obtain the qualitative assessment cell presence and distribution. Once data was collected for sterile twenty-four hour experiments, seven-day culture set-ups could be evaluated. Seven-day set-ups followed the same protocols as twenty-four hour experiments except for the addition of feeding schedules. For longer duration studies, a new conical of fibroblast media was given every other day until the conclusion of the test. 
The non-sterile scaffold test resulted in contamination after cells were introduced into the bioreactor. The contamination caused discoloration of bioreactor and reservoir media (Fig. 44). The experiment had to be taken down and disassembled to be cleaned. All fittings were collected and soaked in bleach for twenty-four hours, while the acrylic housing and tubing were sent to EtO to be sterilized before any more experiments could be conducted. Sterile protocols were now used to avoid incidences such as these.

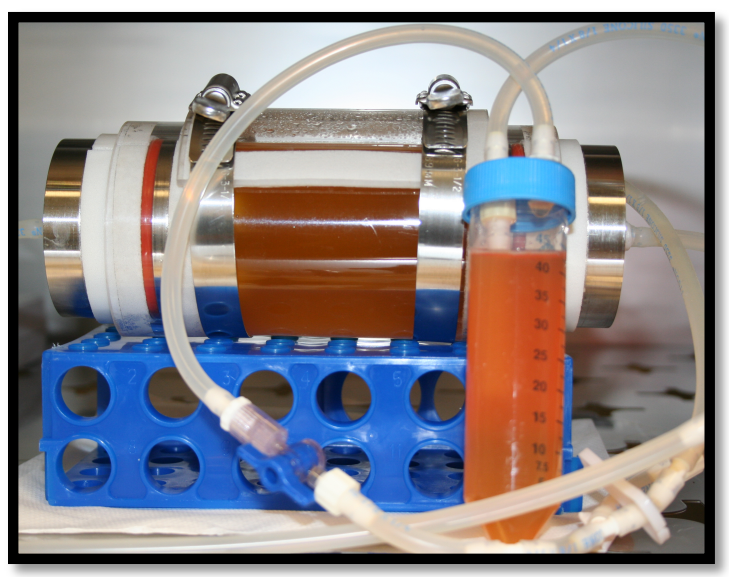

Figure 44: Contaminated bioreactor 7 hours after cells were introduced into the system.

Results from using sterile protocols gave us BBI images of proximal, middle, and distal regions of the scaffold (Fig. 45). These images allowed us to assess consistency and distribution of cells. Contamination was not seen in any cases when sterile protocols were performed. Similar results were seen when performing seven-day culture experiments (Fig. 46). Single-scaffold tests showed that the bioreactor system could culture cells between acute and long-term studies. It was then necessary to perform multi-scaffold tests to assess cell distribution between all scaffolds. 

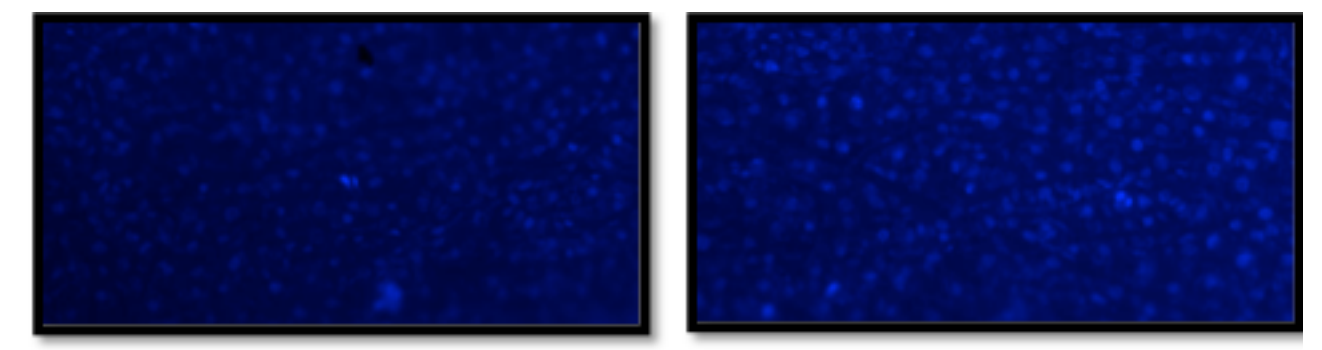

Figure 45: BBI Images of single-scaffold short-term test, showing fibroblasts in proximal (top left), middle (top right), and distal regions (bottom).
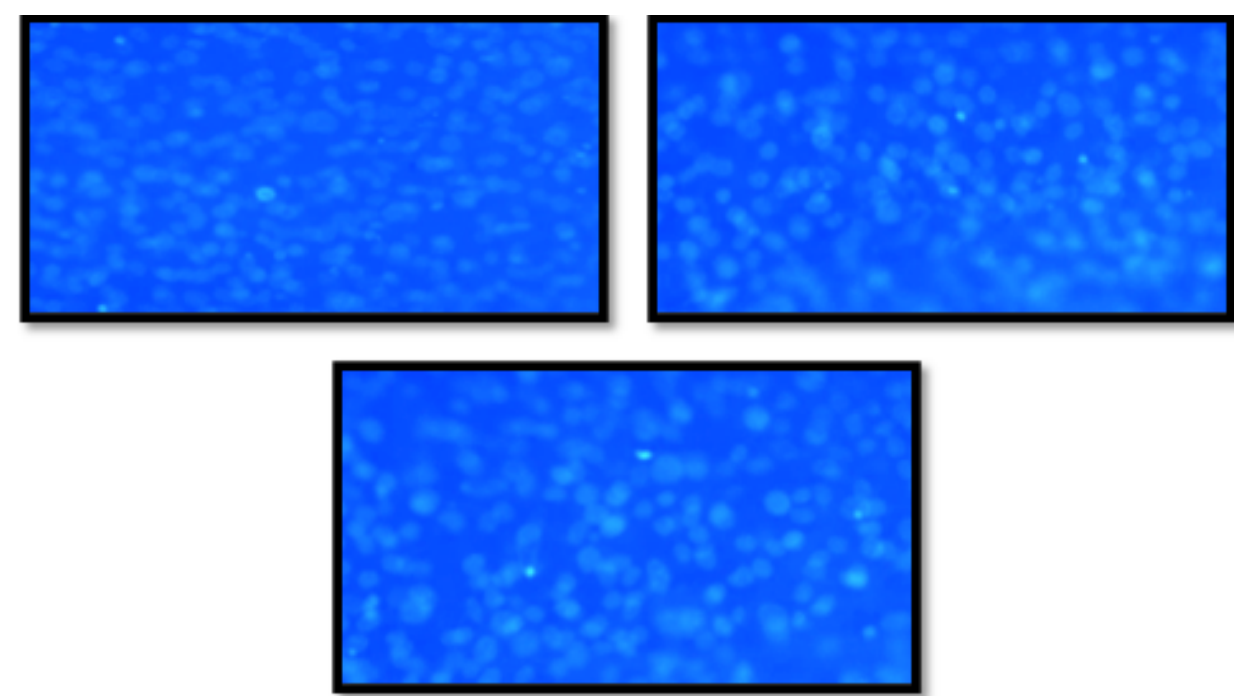

Figure 46: BBI Images of single-scaffold long-term test, showing fibroblasts in proximal (top left), middle (top right), and distal region (bottom).

\subsection{Multiple-Scaffold Testing}

Multiple-scaffold testing provided quantitative results as opposed to the

qualitative results in single scaffold tests. Sterile procedures were used in these experiments, as long-term cultivation (seven days) was required for this stage of testing.

Scaffolds were orientated in a square pattern with each position labeled as scaffold 1-4 
(Fig. 47). Scaffolds 1 and 3 were used to analyze cell distribution from proximal to distal ends. However, the proximal end of scaffolds 2 and the distal end of scaffold 4 were used to run cell viability studies subsequently.

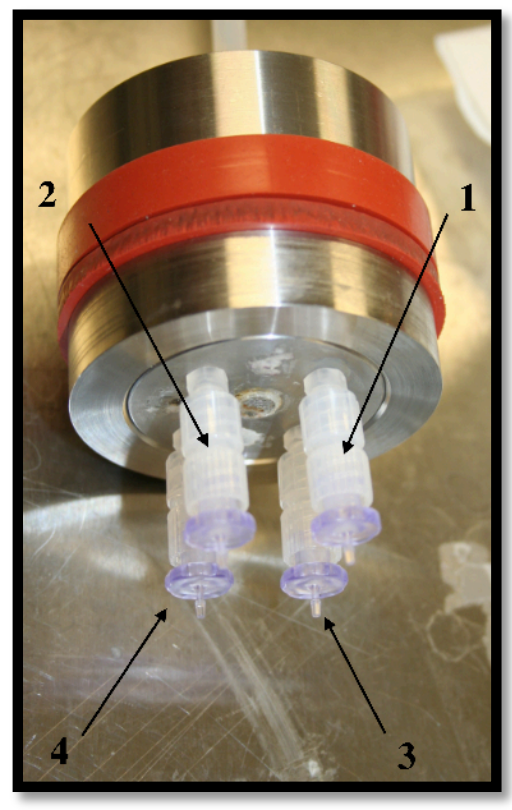

Figure 47: Scaffold configuration of four scaffolds.

The bioreactor went through a twenty-four hour conditioning period under transmural flow prior to sodding of cells. Three T75 flasks of 3T3 fibroblasts grown at $100 \%$ confluency were used to sod the scaffolds, tripling the amount used in singlescaffold experiments. The pump setting was set to setting 1 , giving a flow rate of 4 $\mathrm{mL} / \mathrm{min}$ or a shear stress of 1.4 dynes $/ \mathrm{cm}^{2}$. Fibroblasts were fed according to the same schedule conducted during long-term single-scaffold tests. Cell distribution was assessed through each scaffold region including proximal, middle, and distal, as well as top, bottom, left, and right scaffolds. Cell densities were measured based on average number of cells per $\mathrm{mm}^{2}$ quantified for each region. Three images of each region, proximal, middle, and distal, were taken. Using the Ronchi Ruling, a slide with 150 vertical lines 
per millimeter used to determine the size of the field, a conversion factor from pixels to millimeters was created. A box of known dimensions was then used to gain information on the number of cells inside the box, and three box counts were made per image (Fig. 48).

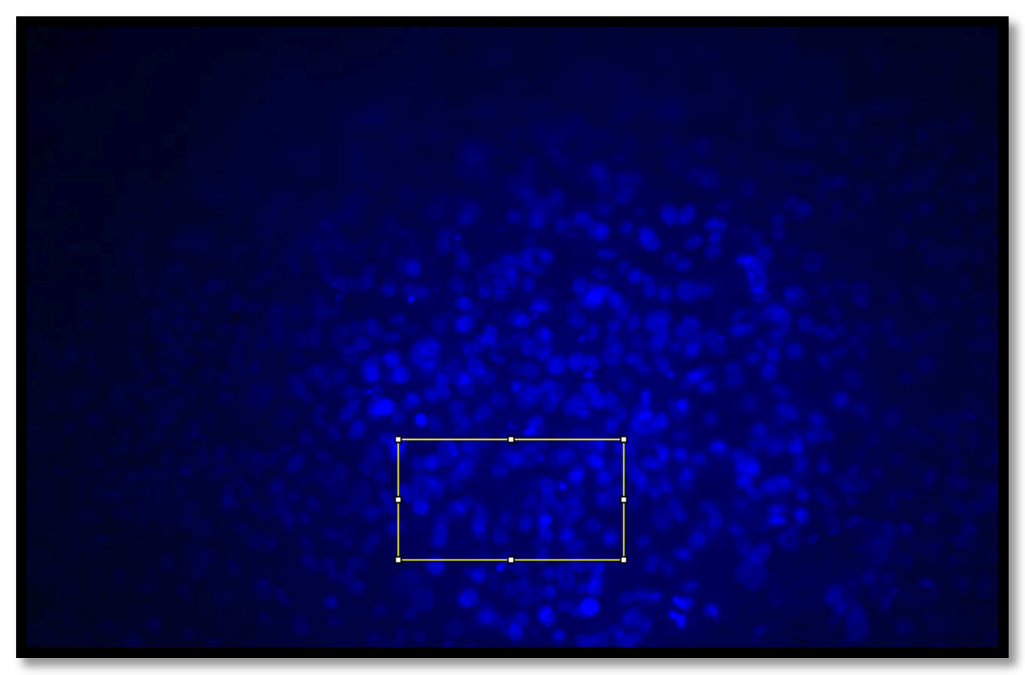

Figure 48: Box created to count cells in each image to obtain cells $/ \mathrm{mm}^{2}$.

Cell densities were calculated per box resulting in number of cells per $\mathrm{mm}^{2}$. By using the raw data, finding the number of cells per $\mathrm{mm}^{2}$, taking the log of those cell densities, and averaging them, assuming the data follows a Poisson distribution, this corrected any lack of equality of spread. Performing a log transformation helped the data to be amenable for analysis of variance (ANOVA) procedures. Essentially averaging the log created a more normal distribution. Data preparation when conducting T-tests used the same technique. The T-tests were used to evaluate total cell number differences between top and bottom scaffolds. ANOVA tests assessed differences in regions of different scaffolds including proximal, middle, and distal. Tukey tests were used in association with ANOVA to figure out which variables were different. 
After seven days of culture with no leaks or contamination, results showed that cells were still present on the inner lumen of the scaffold. These results were used to gain quantitative information regarding differences in cell distribution between the top, bottom, left, and right scaffolds. Statistical analysis evaluated differences between top and bottom, and differences in proximal, middle, and distal regions of each of the four scaffolds. BBI images displayed cell distribution in the three scaffold regions (Fig. 49).
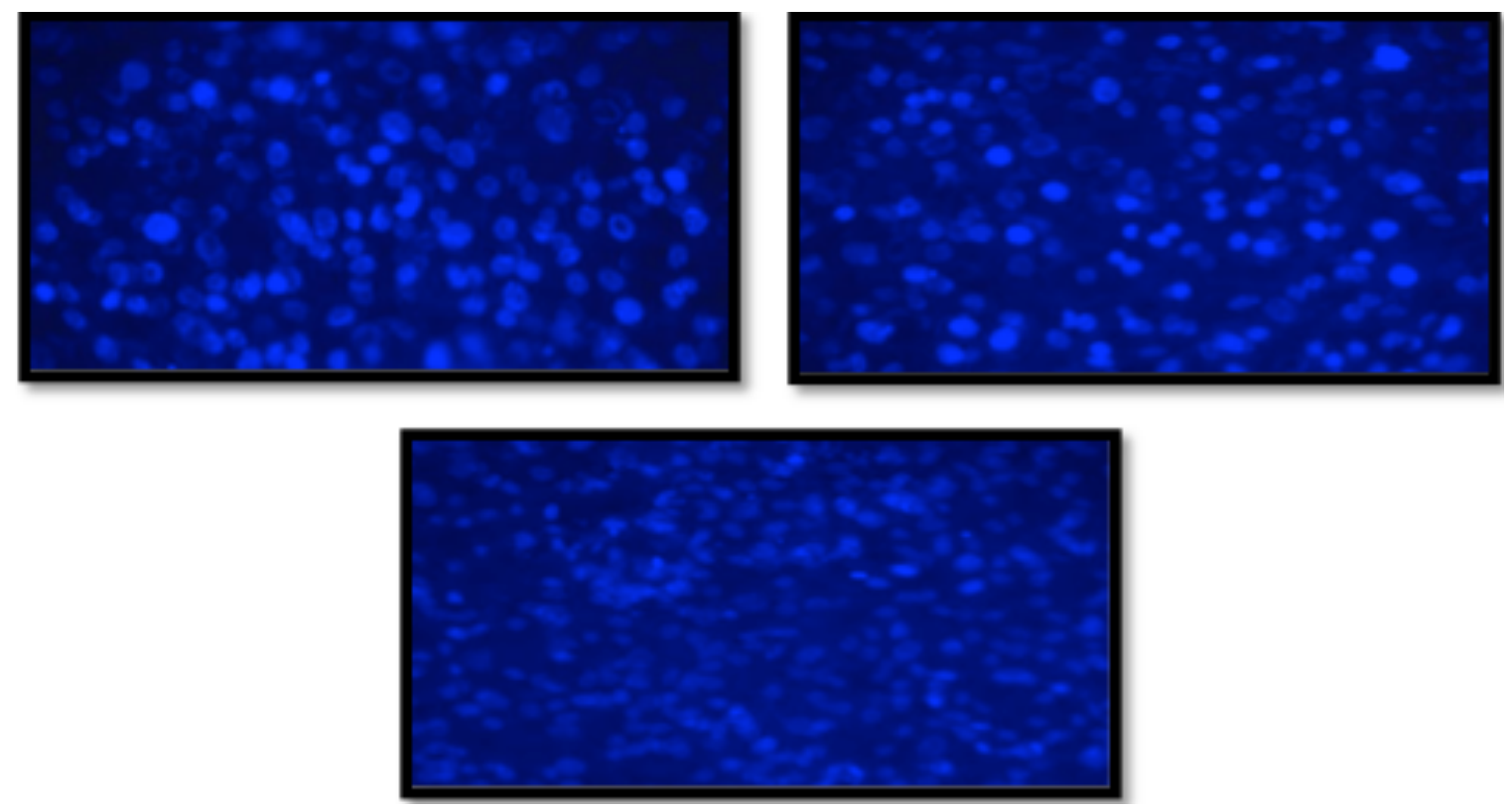

Figure 49: BBI Image of bottom scaffold displaying proximal (top left), middle (top right), and distal (bottom center).

Results of the two-tailed T-test evaluating total cell number per scaffold between top and bottom scaffolds showed a p-value of .0036 , showing that there was a statistical difference in cell density between the top and bottom scaffolds. An ANOVA test used to test differences between regions in the top and bottom scaffolds resulted with a p-value of 0.0355 , further assessing statistical difference in the cell density between various regions of the scaffolds. Finally, a Tukey test indicated no differences between various regions of the scaffold. 
ANOVA procedures were also used to test differences between each proximal region for all scaffolds resulting with a p-value of less than 0.0001 , showing statistical difference in the proximal regions of all the scaffolds. A Tukey test was used to determine which was different (Table VIII). The test indicated that scaffold 3 and 4 were not different from each other, but both were different from scaffold 1 .

Table VIII: Tukey test evaluating which proximal region is different. The column labeled "Mean" refers to the average log of the cellular densities for the proximal regions.

\begin{tabular}{|l|l|r|}
\hline Level & & Mean \\
\hline 4 & $\mathrm{~A}$ & 6.30 \\
\hline 3 & $\mathrm{~A}$ & 6.04 \\
\hline 1 & $\mathrm{~B}$ & 5.51 \\
\hline
\end{tabular}

ANOVA testing on middle regions resulted with a p-value of 0.0013 , indicating that there were statistical differences in the middle regions of all the scaffolds. A Tukey test was utilized to see which middle region was different from the others (Table IX). The results showed that groups with letter that did not overlap were statistically different. Another ANOVA meant to investigate differences between distal regions of different scaffolds produced a p-value of 0.8567 , showing no statistical differences between the distal regions.

Table IX: Tukey test evaluating which middle region is different. The column labeled "Mean" refers to the average log of the cellular densities for the middle regions.

\begin{tabular}{|l|r|r|}
\hline $\mathbf{1}$ & & Mean \\
\hline 3 & A & 6.0878919 \\
\hline 4 & A B & 5.8386302 \\
\hline 1 & B & 5.2678068 \\
\hline 2 & B & 5.2503754 \\
\hline
\end{tabular}


The results obtained from the T-test when evaluating total cell number for both top and bottom scaffolds, indicated that the bottom scaffold had more cells compared to the top (Fig. 50). This could be a result of slow injection rates, resulting in flow toward the path of least resistance. By implementing an additional sodding procedure to the bioreactor, this solution would assess the uneven distribution between the top and bottom scaffolds. Although the Tukey test did not show differences between regions, the ANOVA test did show a difference. This occurred based on the Tukey multiple comparison tests only comparing means, and that the overall $\mathrm{p}$-value produced by the ANOVA does not guarantee that the Tukey test will find a significant difference [66].

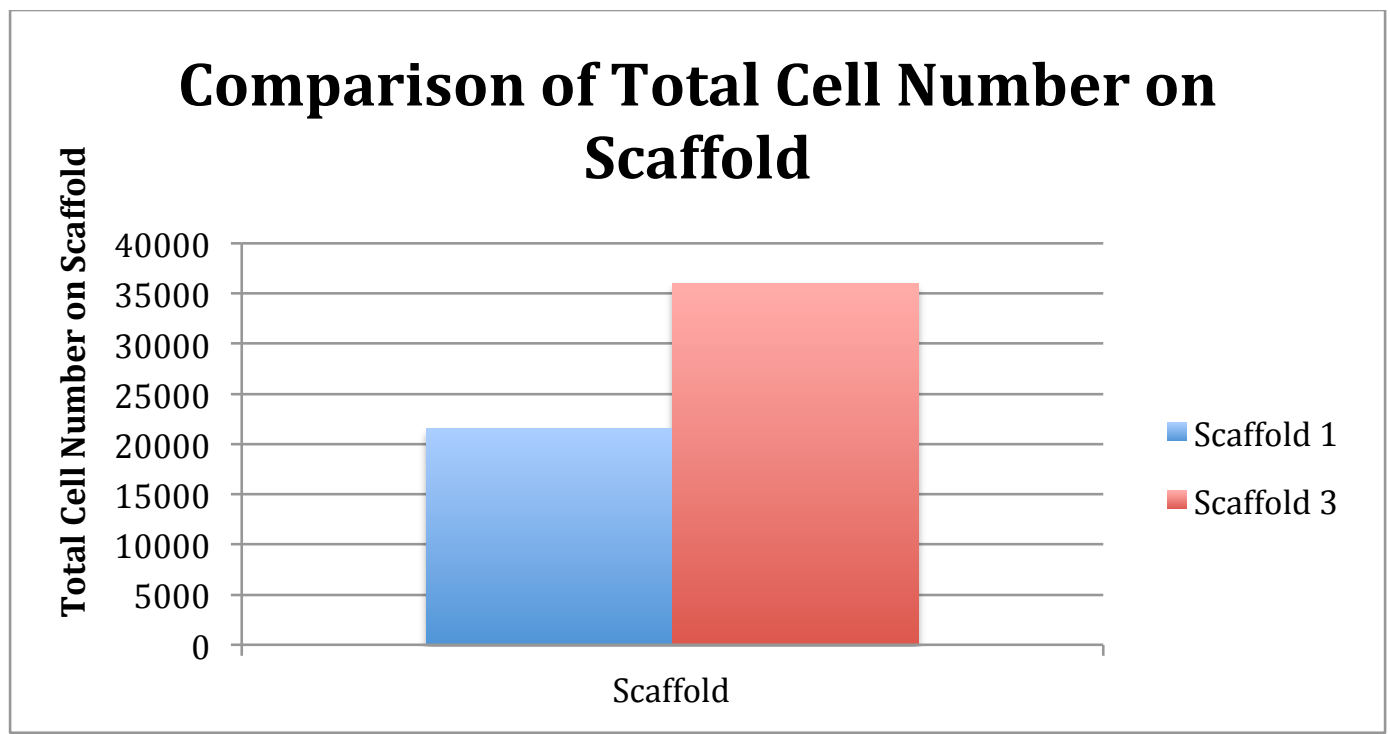

Figure 50: Graph displaying differences in total cell number on scaffold between top and bottom scaffold arrangement.

The ANOVA test that evaluated proximal regions for all scaffolds indicated that there was a difference. The corresponding Tukey test showed that top (scaffold 1) and bottom (scaffold $3 \& 4$ ) proximal regions were different, however no differences could be seen between left and right bottom proximal regions. ANOVA testing of middle regions of the scaffolds showed that there was a difference across all scaffolds. The Tukey tests 
indicated that there was only a difference between the top and bottom on scaffold 3 . The middle region of scaffold 4 showed no difference between the two top scaffolds. There were no differences between left and right scaffolds as well. Lastly, ANOVA testing distal regions across all scaffolds indicated that there were no differences (Fig. 51). In the end, results from the proximal region of scaffold 2 and the distal region of scaffold 4 were excluded from this study and were used in the results of cell viability described in the next section.

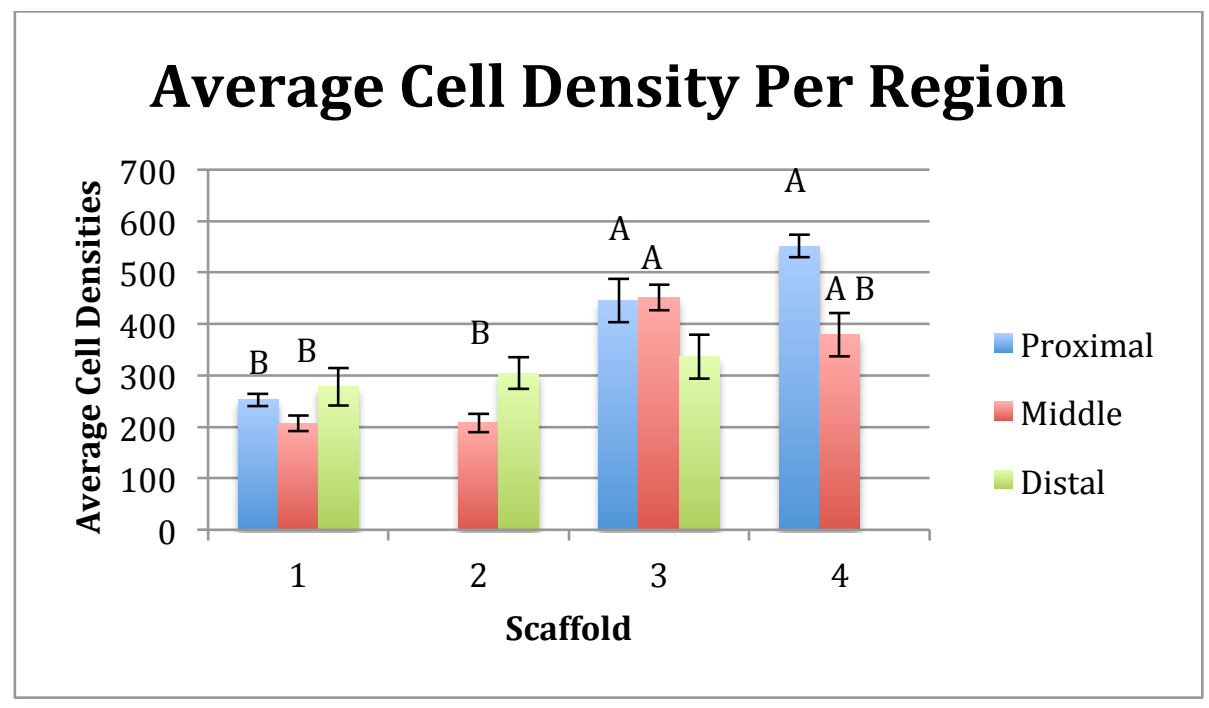

Figure 51: A graph displaying average cell densities versus scaffold and scaffold region. Letters are results of a Tukey tests indicating similarities and differences across scaffold regions. Proximal and distal regions of scaffolds 2 and 4 were excluded as their results were used in cell viability testing.

A trend showing an increase in average cell density from top to bottom scaffolds at the proximal end to the distal end was observed, however there are no differences between regions at the distal end. This may be a result from sodding the cells from one side of the bioreactor. Including an alternative cell induction port to sod cells in a different location would contribute to provide uniform distribution from proximal to distal ends of the scaffolds. 


\subsection{Cell Viability Testing}

Evaluation of cell viability was necessary to illustrate that the system could cultivate a living tissue construct and determine if EtO sterilization was harmful to cells. Cell viability testing used sterile protocols as well as single and multiple-scaffold tests. Culture duration was seven days serving as a good indicator if cells were harmed by EtO residue. Cell culture requirements for both single and multi-scaffold set-ups previously mentioned were used in cell viability testing. A Single-scaffold set-up was first used to assess viability. Multi-scaffold set-ups were used to obtain more cells to analyze. Using the cell viability protocol created by Chris Miracle [69], once the scaffold was extracted from the bioreactor, cells were trypsinized from the scaffold and assessed using either trypan blue or cell viability assay kit provided by Invitrogen (L3224). Trypan blue enters the cells based on its membrane integrity, resulting in live cells with a blue outline around their perimeter and a clear center, and dead cells colored blue. The cell viability assay kit stains living cells green and dead cells red, and utilizes a hemocytometer to perform counts. Protocols for cell viability tests are included in Appendix D.

Cell viability results could not be obtained in single-scaffold tests due to the small number of cells that could possibly be extracted from the scaffold. When multi-scaffold set-ups were used, results provided cell viability percentages. Table $\mathrm{X}$ shows the different measurements taken from the cell counter (Nexcelcom Cellometer Auto T4). As some quadrants were absent of cells, an automated count could not be obtained and manual counts were made. No dead cells were found in any of the quadrants of the cell counter slide. To verify these results, images taken on the fluorescent microscope indicated living and dead cells (Fig. 52). 
Table X: Table showing results obtained from cell counter. All counts resulted in $100 \%$ viability with no visual indication of dead cells in any of the quadrants of the cell counter slide.

\begin{tabular}{|c|c|}
\hline Cell Viability & Total Cell Concentration $($ Cells $/ \mathrm{ml})$ \\
\hline $100 \%$ & $8.8 \times 10^{4}$ \\
\hline $100 \%$ & $8.5 \times 10^{4}$ \\
\hline $100 \%$ & $9.9 \times 10^{4}$ \\
\hline $100 \%$ & $1.3 \times 10^{5}$ \\
\hline $100 \%$ & $3.9 \times 10^{4}$ \\
\hline
\end{tabular}
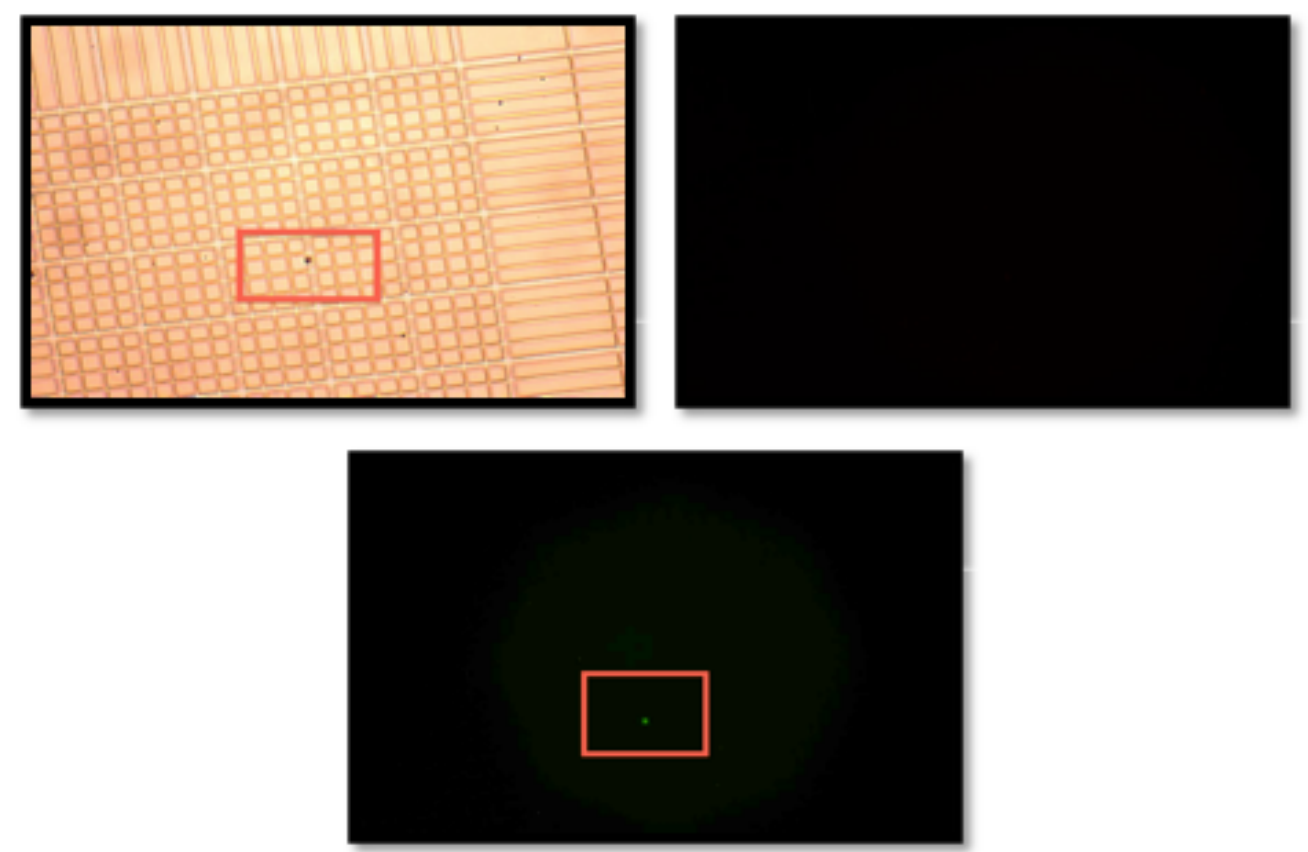

Figure 52: One set of images from fluorescent microscope indicating no dead cells observed. The cell highlighted inside the red box indicates the living cell found in that region of the hemocytometer.

Evaluation of the cell viability studies concluded that EtO residue was not harmful to cells and cell viability in long-term culture was achievable. The results obtained from the cell counter cannot conclusively show that there was $100 \%$ cell viability. Cell viability is a percentage that requires both live and dead cell counts, and as there were no visible dead cells, the percentage reflects only the live cells counted. Additional testing is required to fully verify the $100 \%$ cell viability figure. Although few living cells were 
found on the hemocytometer, no dead cells were observed. The small amount of cells present on the hemocytometer may have been a result of the design of the experiment: when assessing cell viability, only halves of scaffolds were used. Thus, when the cells were trypsinized there may have been a low number of cells obtained, resulting in a small pellet when centrifuged or accidently aspirated when removing the supernatant. However, results did show the presence of living cells and absence of dead cells. From these results, pre-installation of scaffolds prior to sterilization was safe to perform, and allowed for a decreased set-up time of fifteen minutes in the hood. The studies performed above in this segment of work illustrated that the bioreactor could perform as well as the other culture system seen in the laboratory. The bioreactor's ability to perform these cellular tests allows the introduction of native components of the BBB in future testing. 


\section{Chapter 6: Final Discussion, Future Work, Limitations, \& Conclusion}

\subsection{Final Discussion}

Overall, the design of the bioreactor was optimized to display ease of use in assembly, sterilization, conditioning, and culture. Assembly was developed so that the user had no complications when inserting the outer caps into the housing. The method of pre-scaffold installation allowed more space for users to work with the bioreactor, provided less concern about using aseptic technique, and translated to easier set up procedures when conditioning the bioreactor. This method led to the protocol of never having to interact with the internal components of the bioreactor once sterilized, sustaining sterile conditions and creating easier protocols for use.

Placing the bioreactor in one package allowed the system to be fully assembled and allowed the system to require less time in the hood. After determining that contamination can occur when tubing, scaffolds, and components are not sterilized in non-sterile 24-hour experiments, sterile procedures were chosen to be apart of all experimental set-ups when working with this bioreactor system. As a result, there was only one contamination occurrence out of all the experiments conducted when testing the system.

The flow rates measured and used in the bioreactor system were similar to rates measured in literature $[53,57,60]$. For example, Cucullo et al., showed that shear stress maximum in blood capillaries was about 5 dynes $/ \mathrm{cm}^{2}$ [53]. All of the experiments were well under this estimated maximum shear stress. During testing of a new DIV-BBB, Cucullo discussed that the pumping mechanism was capable of generating flow levels from 1 to $50 \mathrm{ml} / \mathrm{min}$ with associated shear stresses of 1-200 dynes $/ \mathrm{cm}^{2}$ [58]. These ranges 
were achieved with the system presented here as well. Compared to the flow rates measured in Mr. Brandon's project, similar flow rates were achieved providing suitable ranges of shear stresses for future testing on the bioreactor.

The bioreactor designed in this thesis aimed to improve upon the limitations of the CellMax system, including the destruction of the CellMax bioreactors and the difficulty of extracting scaffolds. The bioreactor was designed to be reusable, avoiding the need to purchase multiple bioreactors for each experiment. The bioreactor cap allowed access into the bioreactor chamber, preserving the bioreactor during scaffold extraction. The use of an acrylic housing allowed increased visibility into the bioreactor chamber to monitor the scaffolds and the media during culture. Although, the size and weight of the bioreactor were significantly larger than the CellMax system, the bioreactor was able to fit into the allotted space in the large incubator.

Compared to the Lock N Lock bioreactors, the bioreactor used for BBB testing displayed both favorable and non-favorable attributes including assembly processes, chamber access, and sterilization procedures. The BBB bioreactor consists of more components for the users to construct and requires larger packaging for sterilization. The Lock N Lock system consists of a chamber with tubing permanently installed simplifying assembly of the system to connecting the media reservoir and Tupperware cap connections. Assembly time was shorter for the Lock N Lock system compared to the BBB bioreactor. Packaging the BBB bioreactor for sterilization requires the system be fully assembled and partially closed, while the Lock N Lock bioreactors require separate packaging and sterilization procedures for system sterilization. Chamber access was more time intensive in the BBB bioreactors as the hose clamps were removed before opening 
the bioreactor cap, compared to the Lock N Lock bioreactors that required flipping the clamps connected to the cap to access the bioreactor chamber. This eases the process of loading bioreactor media into the bioreactor chamber of the Lock N Locks, and is accomplished by simply pouring media into the bioreactor chamber of the Lock N Locks, instead of using a glass funnel to pour the media into the chamber. The glass funnel technique does require more time to fill the chamber, however was effective in maintaining sterility of the system. The BBB bioreactor's ability to culture more scaffolds at one time can easily be implemented into the Lock N Lock bioreactor with the installation of holes for additional ports. Although the BBB bioreactor can only match some attributes of the Lock N Lock bioreactors, future work can potentially increase the performance of the bioreactor developed in this thesis.

\subsection{Future Work \& Limitations}

To alleviate the problem of non-uniform distribution between top and bottom scaffolds, sodding of cells can also occur when flipping the bioreactor over so that the bioreactor cap is facing down. By introducing a three-way stop cock on the outlet and sodding cells from both sides we could possibly alleviate the problem of uneven distribution at the proximal sides. Additional cell viability tests must be conducted to further evaluate cell viability. The protocol explained (in Section 5.5) can be used to repeat the test, but an additional step of saving the scaffold to observe if all the cells were taken off using BBI should be included. An alternative test could be a static cell viability test with the use of well plates to investigate cell viability on a scaffold. This approach alleviates the need to extract cells from the scaffold. By placing a sample of PLGA at the bottom of a well plate and seeding cells onto the scaffold, the Invitrogen Cell Viability 
Assay can be used to view the cell under the fluorescent microscope after 7 days of culture. Introduction of ECs and glial cells can now be implemented to characterize the interactions between the two cell types. Development of protocols for introducing two cell types into the bioreactor must be made. The creation of smaller barbs could allow for the use of smaller scaffolds, which would provide a more accurate culture environment. The work of two undergraduates, Matt Rogers and Nathan Ferrier, created barb-to-thread fittings that would allow for the use of $1 \mathrm{~mm}$ inner diameter scaffolds in future testing of the bioreactor. However as we scale down even further in size, difficulty increases in protocols such as scaffold cutting during analysis. An assessment must be made as to how protocols for this bioreactor will change as we try to improve upon the recapitulation of the BBB's environment. Concerns such as change in flow rate, scale of the pumping mechanism, culture protocols, and scale of associated components of the bioreactor are brought to attention as well when thinking of introducing more physiological conditions. Using smaller diameter scaffolds will require smaller scale barbs to connect them to. Flow rates that use these smaller barbs and scaffolds will need to be characterized to maintain the shear rate range required for BBB research. If the pump currently being used is not compatible with these smaller barbs and scaffolds to produce these shear ranges, then a new pump will need to be acquired for future tests. Culture protocols might be changed due to the fragility of the scaffolds at such low diameters.

One limitation of this thesis is using a protocol that relies on the use of other departments such as the Animal Science Department for their sterilization procedures. The fact that experiments were constrained to availability for sterilization increases the length of the experiment as a whole. Obtaining an EtO sterilizer for Cal Poly's Tissue 
Engineering lab would allow tests to be conducted at any time. The procedure of trypsinizing the scaffolds during cell viability tests was challenging, limiting the number of cells that could be extracted and viewed under the fluorescent scope. When dealing with cells from only a half of a scaffold, there is only a small volume of cells that can be extracted. There was extreme caution not to aspirate the pellet after centrifugation, as well as not to dilute the pellet during resuspension. By implementing the additional cell viability test mentioned above, the effect of EtO residue on cells could be further explored. Another limitation was the weight of the bioreactor. Although stainless steel was an optimal material to be used in the design of the bioreactor, the weight did cause some accidents when dropped or ripped during packaging. Using a different material may be a solution to reduce the weight of the system, however, durability was chosen over weight to maintain reusability.

\subsection{Conclusion}

The work accomplished in this thesis aimed to build upon the second aim in Mr. Brandon's project [62]. The development and creation of an in-house bioreactor was completed to dramatically reduce costs in testing in the lab, to provide easy access to scaffolds, and to avoid destruction of the bioreactor for assessment and analysis as reiterated in the future work first presented by Mr. Brandon [62]. The aims, design processes, methods, and results of the BBB bioreactor have been discussed in detail throughout this thesis. The creation of this bioreactor has the potential to contribute in the investigations of the BBB and to aid the pharmaceutical industry in the over one billion dollar drug discovery process [70]. The BBB bioreactor provides an in-house, reusable model to recapitulate the BBB compared to previous commercial products. The design of 
the bioreactor helps users to easily assemble, culture, and analyze tissue constructs grown to further increase progress in research and industry. As researchers continue to search for robust systems to recreate physiological environments, this thesis can provide a basis to further develop and improve upon models of the BBB. 


\section{Bibliography}

1. Cardoso, Filipa L. "Looking at the Blood-brain Barrier: Molecular Anatomy and Possible Investigation Approaches." Brain Research Reviews 64 (2010): 328-63. Print.

2. Petty, M.A., Lo, E.H., 2002. Junctional complexes of the blood-brain barrier: permeability changes in neuroinflammation. Prog. Neurobiol. 68, 311-323.

3. Lee, S.W., Kim, W.J., Park, J.A., Choi, Y.K., Kwon, Y.W., Kim, K.W., 2006. Blood-brain barrier interfaces and brain tumors. Arch. Pharm. Res. 29, 265-275.

4. Persidsky, Y., Ramirez, S.H., Haorah, J., Kanmogne, G.D., 2006a. Blood-brain barrier: structural components and function under physiologic and pathologic conditions. J. Neuroimmune Pharmacol. 1, 223-236.

5. Pardridge, W.M., 1999. Blood-brain barrier biology and methodology. J. Neurovirol. 5, 556-569.

6. Hawkins, B.T., Egleton, R.D., 2006. Fluorescence imaging of blood-brain barrier disruption. J. Neurosci. Methods 151, 262-267.

7. Khan, E., 2005. An examination of the blood-brain barrier in health and disease. Br. J. Nurs. 14, 509-513

8. Choi, Y.K., Kim, K.W., 2008. Blood-neural barrier: its diversity and coordinated cell-to-cell communication. BMB Rep. 41, 345-352

9. Abbott, N.J., Ronnback, L., Hansson, E., 2006. Astrocyte-endothelial interactions at the blood-brain barrier. Nat. Rev. Neurosci. 7, 41-53.

10. Zlokovic, B.V., 2008. The blood-brain barrier in health and chronic neurodegenerative disorders. Neuron 57, 178-201.

11. Weiss, N., Miller, F., Cazaubon, S., Couraud, P.O., 2009. The blood-brain barrier in brain homeostasis and neurological diseases. Biochim. Biophys. Acta 1788, 842-857.

12. Chaudhuri, J.D., 2000. Blood brain barrier and infection. Med. Sci. Monit. 6, $1213-1222$

13. De Boer, A.G., Gaillard, P.J., 2006. Blood-brain barrier dysfunction and recovery. J. Neural Transm. 113, 455-462.

14. Kumar, P., Shen, Q., Pivetti, C.D., Lee, E.S., Wu, M.H., Yuan, S.Y., 2009. Molecular mechanisms of endothelial hyperpermeability: implications in inflammation. Expert Rev. Mol. Med. 30, 11:e19

15. Wolburg, H., Noell, S., Mack, A., Wolburg-Buchholz, K., Fallier-Becker, P., 2009. Brain endothelial cells and the glio-vascular complex. Cell Tissue Res. 335, $75-96$.

16. Lee, S.J., Benveniste, E.N., 1999. Adhesion molecule expression and regulation on cells of the central nervous system. J. Neuroimmunol. 98, 77-88.

17. Carvey, P.M., Hendey, B., Monahan, A.J., 2009. The blood-brain barrier in neurodegenerative disease: a rhetorical perspective. J. Neurochem. 111, 291-314.

18. Adibhatla, R.M., Hatcher, J.F., 2008. Tissue plasminogen activator (tPA) and matrix metalloproteinases in the pathogenesis of stroke: therapeutic strategies. CNS Neurol. Disord. Drug Targets 7, 243-253.

19. Kim, J.A., Tran, N.D., Li, Z., Yang, F., Zhou, W., Fisher, M.J., 2006. Brain endothelial hemostasis regulation by pericytes. J. Cereb. Blood Flow Metab. 26, $209-217$ 
20. Lai, C.H., Kuo, K.H., 2005. The critical component to establish in vitro BBB model: pericyte. Brain Res. Brain Res. Rev. 50, 258-265.

21. Allt, G., Lawrenson, J.G., 2001. Pericytes: cell biology and pathology. Cells Tissues Organs 169, 1-11.

22. Bagley, R.G., Weber, W., Rouleau, C., Teicher, B.A., 2005. Pericytes and endothelial precursor cells: cellular interactions and contributions to malignancy. Cancer Res. 65, 9741-9750.

23. Hawkins, B.T., Davis, T.P., 2005. The blood-brain barrier/neurovascular unit in health and disease. Pharmacol. Rev. 57, 173-185.

24. Colgan, O.C., Collins, N.T., Ferguson, G., Murphy, R.P., Birney, Y.A., Cahill, P.A., Cummins, P.M., 2008. Influence of basolateral condition on the regulation of brain microvascular endothelial tight junction properties and barrier function. Brain Res. 1193, 84-92.

25. Satoh J., Tabunoki H., Yamamura T., Arima K. and Konno H. (2007) Human astrocytes express aquaporin-1 and aquaporin-4 in vitro and in vivo. Neuropathology 27, 245-256.

26. Choi, Y.K., Kim, K.W., 2008. Blood-neural barrier: its diversity and coordinated cell-to-cell communication. BMB Rep. 41, 345-352.

27. Lundquist, Stefan. "The Use of in Vitro Cell Culture Models for Mechanistic Studies and as Permeability Screens for the Blood-brain Barrier in the Pharmaceutical Industry - Background and Current Status in the Drug Discovery Process." Vascular Pharmacology 38 (2002): 355-64. Print

28. Eddy, E. "In Vitro Models to Predict Blood-brain Barrier Permeability." Advanced Drug Delivery Reviews 23.1-3 (1997): 185-98. Print.

29. Terasaki, T. "New Approaches to in Vitro Models of Blood-brain Barrier Drug Transport." Drug Discovery Today 8.20 (2003): 944-54. Print.

30. Van Vliet, E.A., da Costa Araújo, S., Redeker, S., van Schaik, R., Aronica, E., Gorter, J.A., 2007a. Blood-brain barrier leakage may lead to progression of temporal lobe epilepsy. Brain 130, 521-534.

31. Culot, Maxime, Stefan Lundquist, Dorothée Vanuxeem, Stéphane Nion, Christophe Landry, Yannick Delplace, Marie-Pierre Dehouck, Vincent Berezowski, Laurence Fenart, and Roméo Cecchelli. "An in Vitro Blood-brain Barrier Model for High Throughput (HTS) Toxicological Screening." Toxicology in Vitro 22.3 (2008): 799-811. Print.

32. Reichel, Andreas. "The Role of Blood-Brain Barrier Studies in the Pharmaceutical Industry." Current Drug Metabolism 7.2 (2006): 183-203. Print.

33. Zimmer, Carl. "The Brain - Maybe You Do Need a Hole in Your Head to Let the Medicine In." Discover Magazine 15 Nov. 2011. Web. 5 Feb. 2012

34. Hasselbalch, S.G., Knudsen, G.M., Capaldo, B., Postiglione, A., Paulson, O.B., 2001. Blood-brain barrier transport and brain metabolism of glucose during acute hyperglycemia in humans. J. Clin. Endocrinol. Metab. 86, 1986-1990.

35. Reinhard, M., Hetzel, A., Krüger, S., Talazko, J., Ziyeh, S., Weber, J., Els, T., 2006. Blood-brain barrier disruption by low-frequency ultrasound. Stroke 37, 1546-1548.

36. Kusch-Poddar, Manisha. In Vitro Models of the Blood-Brain Barrier: Applications and Evaluation of a New Human Immortalized Brain Capillary 
Endothelial Cell Line. Diss. Philosophisch-Naturwissenschaftlichen Fakultat Der Universitat Basel, 2006. Print.

37. Bickel, U. "How to Measure Drug Transport across the Blood-Brain Barrier." NeuroRX2.1 (2005): 15-26. Print.

38. Lemaire, M., and S. Desrayaud. "The Priorities/needs of the Pharmaceutical Industry in Drug Delivery to the Brain." International Congress Series 1277 (2005): 32-46. Print

39. Avdeef, Alex. "How Well Can in Vitro Brain Microcapillary Endothelial Cell Models Predict Rodent in Vivo Blood-brain Barrier Permeability?" European Journal of Pharmaceutical Sciences 43.3 (2011): 109-24. Print.

40. Demeuse, P., A. Kerkhofs, C. Struysponsar, B. Knoops, C. Remacle, and P. Vandenboschdeaguilar. "Compartmentalized Coculture of Rat Brain Endothelial Cells and Astrocytes: A Syngenic Model to Study the Blood-brain Barrier."Journal of Neuroscience Methods 121.1 (2002): 21-31. Print.

41. Dagenais, Claude, Alex Avdeef, Oksana Tsinman, Adam Dudley, and Richard Beliveau. "P-glycoprotein Deficient Mouse in Situ Blood-brain Barrier Permeability and Its Prediction Using an in Combo PAMPA Model." European Journal of Pharmaceutical Sciences 38.2 (2009): 121-37. Print

42. Egleton, R.D., Mitchell, S.A., Huber, J.D., Palian, M.M., Polt, R., Davis, T.P., 2001. Improved blood-brain barrier penetration and enhanced analgesia of an opioid peptide by glycosylation. J. Pharmacol. Exp. Ther. 299, 967-972.

43. Walker, I. "The Blood-brain Barrier: In Vitro Methods and Toxicological Applications."Toxicology in Vitro 9.2 (1995): 191-204. Print.

44. Lossi, L., Alasia, S., Salio, C., Merighi, A., 2009. Cell death and proliferation in acute slices and organotypic cultures of mammalian CNS. Prog. Neurobiol. 88, 221-245.

45. Cho, S., Wood, A., Bowlby, M.R., 2007. Brain slices as models for neurodegenerative disease and screening platforms to identify novel therapeutics. Curr. Neuropharmacol. 5, 19-33.

46. Harris, L.W., Wayland, M., Lan, M., Ryan, M., Giger, T., Lockstone, H., Wuethrich, I., Mimmack, M., Wang, L., Kotter, M., Craddock, R., Bahn, S., 2008. The cerebral microvasculature in schizophrenia: a laser capture microdissection study. PLoS ONE 3, e3964.

47. Crecelius, A., Götz, A., Arzberger, T., Arnold, G.J., Ferrer, I., Kretzschmar, H.A., 2008. Assessing quantitative post-mortem changes in the gray matter of the human frontal cortex proteome by 2-D DIGE. Proteomics 8, 1276-1291.

48. Mehdipour, Ahmad Reza, and Mehrdad Hamidi. "Brain Drug Targeting: A Computational Approach for Overcoming Blood-brain Barrier." Drug Discovery Today (2009): n. pag. Print.

49. D.E. Clark, In silico prediction of blood-brain barrier permeation, Drug Discov. Today 8 (20) (2003) 927-973.

50. M. Lobell, L. Molnar, G.M. Keseru, Recent advances in the prediction of bloodbrain partitioning from molecular structure, J. Pharm. Sci. 92 (2) (2003) 360-370.

51. X. Liu, et al., Development of a computational approach to predict blood-brain barrier permeability, Drug Met. Dispos. 32 (1) (2004) 132-139. 
52. Chen, Yan, Qing-Jun Zhu, Jian Pan, Yi Yang, and Xiao-Pei Wu. "A Prediction Model for Blood-brain Barrier Permeation and Analysis on Its Parameter Biologically."Computer Methods and Programs in Biomedicine 95.3 (2009): 28087. Print

53. Cucullo, Luca. "Drug Delivery and in Vitro Models of the Blood-brain Barrier." Current Opinion in Drug Discovery \& Development 8.1 (2005). Print.

54. Lauer, R. "Development of an in Vitro Blood-brain Barrier Model Based on Immortalized Porcine Brain Microvascular Endothelial Cells." Il Farmaco 59.2 (2004): 133-37. Print.

55. Hatherell, Kathryn, Pierre-Olivier Couraud, Ignacio A. Romero, Babette Weksler, and Geoffrey J. Pilkington. "Development of a Three-dimensional, All-human in

56. Santaguida, Stefano, Damir Janigro, Mohammed Hossain, Emily Oby, Edward Rapp, and Luca Cucullo. "Side by Side Comparison between Dynamic versus Static Models of Blood-brain Barrier in Vitro: A Permeability Study." Brain Research 1109.1 (2006): 1-13. Print.

57. Stanness, Kathe A. "A Dynamic Model Of The Blood-Brain Barrier "In Vitro""NeuroToxicology 17.2 (1996): 481-96. Print.

58. Cucullo, Luca, Mohammed Hossain, Ed Rapp, Toby Manders, Nicola Marchi, and Damir Janigro. "Development of a Humanized In Vitro Blood?Brain Barrier Model to Screen for Brain Penetration of Antiepileptic Drugs." Epilepsia 48.3 (2007): 505-16. Print.

59. Stanness, Kathe A., Lesnick E. Westrum, Eleonora Fornaciari, Patrizia Mascagni, Jay A. Nelson, Stephan G. Stenglein, Tim Myers, and Damir Janigro.

"Morphological and Functional Characterization of an in Vitro Blood-brain Barrier Model." Brain Research 771.2 (1997): 329-42. Print.

60. Neuhaus, Winfried, Regina Lauer, Silvester Oelzant, Urs P. Fringeli, Gerhard F. Ecker, and Christian R. Noe. "A Novel Flow Based Hollow-fiber Blood-brain Barrier in Vitro Model with Immortalised Cell Line PBMEC/C1-2." Journal of Biotechnology 125.1 (2006): 127-41.Print

61. Dehouck, M.p., B. Dehouck, C. Schluep, M. Lemaire, and R. Cecchelli. "Drug Transport to the Brain: Comparison between in Vitro and in Vivo Models of the Blood-brain Barrier." European Journal of Pharmaceutical Sciences 3.6 (1995): 357-65. Print

62. Brandon, Bryan. Developing a Dynamic in Vitro Blood-Brain Barrier Model. Diss. California Polytechnic State University, 2010. San Luis Obispo: California Polytechnic State University, 2010. Print.

63. Mahaffey, Ian. A Novel In-House Design of a Bioreactor for the Modeling of an in Vitro Blood Brain Barrier Model. A Novel In-House Design of a Bioreactor for the Modeling of an in Vitro Blood Brain Barrier Model. California Polytechnic State University, 10 June 2011. Web.

64. Spectrum Laboratories, I. 2010 July 3, 2010]; Available from: http://www.spectrumlabs.com/cell/CellCulturing.html.

65. Rubin, L. L. "A Cell Culture Model of the Blood-brain Barrier." The Journal of Cell Biology 115.6 (1991): 1725-735. Print.

66. Patel, Deven C. Preparation and Characterization of Electrospun PLGA Scaffolds for Vascular Tissue Engineering and the Advancement of an In Vitro 
Blood-Brain Barrier Model. Thesis. California Polytechnic State University, 2012. San Luis Obispo: California Polytechnic State University, 2012. Print.

67. Holy, Chantal E. "Optimizing the Sterilization of PLGA Scaffolds for Use in Tissue Engineering." Biomaterials 22.1 (2000): 25-31. Print.

68. "One-way ANOVA Computes a P Value." GraphPad. GraphPad, 2002. Web. 01 July 2012. <http://www.graphpad.com/faq/viewfaq.cfm?faq=782>.

69. Miracle, Chris. Assay Development for the Assessment of Endothelial Cell Adhesion on Polymeric Scaffolds for Tissue Engineered Blood Vessel Mimics. Final Project. California Polytechnic State University, n.d. San Luis Obispo: California Polytechnic State University, n.d. Print.

70. "Inside Drug Discovery." Innovation.org -. N.p., n.d. Web. 01 July 2012. $<$ http://www.innovation.org/index.cfm/insidedrugdiscovery $>$. 


\title{
Appendix A - Abbreviations
}

\author{
AIDS - Acquired Immune Deficiency Syndrome \\ AJs - Adherens Junction Proteins \\ ANOVA - Analysis of Variance \\ BBB - Blood-Brain Barrier \\ BBI - Bisbenzimide \\ BMVEC - Brain Microvascular Endothelial Cells \\ BUI - Brain Uptake Index \\ CNS - Central Nervous System \\ CSF - Cerebral-Spinal Fluid \\ DIV-BBB - Dynamic In Vitro Model of the Blood-Brain Barrier \\ ECs - Endothelial Cells \\ ePTFE - Expanded Polytetrafluoroethylene \\ EtO - Ethylene Oxide \\ HTS - High Throughput Screening \\ NVU - Neurovascular Unit \\ PET - Positron Emission Tomography \\ PLGA - Poly(lactic-co-glycolic acid) \\ QSAR - Quantitative Structure Activity Relationship \\ TEER - Trans-endothelial electrical resistance \\ TJs - Tight Junctions
}




\section{Appendix B - Parts Information and Design Drawings}

\begin{tabular}{|c|c|c|c|c|}
\hline Component & Description & Company & Website & $\begin{array}{l}\text { Part } \\
\text { Num } \\
\text { ber }\end{array}$ \\
\hline $\begin{array}{l}\text { Outer } \\
\text { Housing }\end{array}$ & $\begin{array}{l}\text { Scratch-Resistant Clear } \\
\text { Cast Acrylic Tube, } \\
\text { 3" OD X 2-5/8" ID, } \\
\text { 1' Length }\end{array}$ & McMaster-Carr & www.mcmaster.com & $\begin{array}{l}8486 \\
K 463\end{array}$ \\
\hline Outer Caps & 2.497” DIA Outer Caps & $\begin{array}{l}\text { Atech } \\
\text { Manufacturing }\end{array}$ & & $\mathrm{N} / \mathrm{A}$ \\
\hline $\begin{array}{l}\text { Bioreactor } \\
\text { Cap }\end{array}$ & $\begin{array}{l}\text { Scratch-Resistant Clear } \\
\text { Cast Acrylic Tube, 3- } \\
\text { 1/2" OD X 3-1/8" ID, 1' } \\
\text { Length }\end{array}$ & McMaster-Carr & www.mcmaster.com & $\begin{array}{l}8486 \\
K 473\end{array}$ \\
\hline Silicon Sheet & $\begin{array}{l}\text { Super-Soft Silicone } \\
\text { Rubber, Adhesive Back, } \\
\text { 1/16" Thick, 12" X12", } \\
\text { 20A Durometer }\end{array}$ & McMaster-Carr & www.mcmaster.com & $\begin{array}{l}9010 \\
\text { K422 }\end{array}$ \\
\hline $\begin{array}{l}\text { Internal O- } \\
\text { ring }\end{array}$ & $\begin{array}{l}\text { Silicone Sealing Washer } \\
\text { 1" Screw Size, 2" OD, } \\
.093 \text { " Thick, Packs of } 10\end{array}$ & McMaster-Carr & Www.mcmaster.com & $\begin{array}{l}9960 \\
4 \mathrm{~A} 13 \\
1 \\
\end{array}$ \\
\hline $\begin{array}{l}\text { Custom } \\
\text { Square O- } \\
\text { ring }\end{array}$ & $\begin{array}{l}\text { Super-Soft Silicone } \\
\text { Rubber, Plain Back, } \\
\text { 3/16" Thick, 6" X 6", } \\
\text { 20A Durometer }\end{array}$ & McMaster-Carr & www.mcmaster.com & $\begin{array}{l}9010 \\
\mathrm{~K} 842\end{array}$ \\
\hline Scaffold Cap & $\begin{array}{l}1.75 ” \text { DIA Scaffold } \\
\text { Caps }\end{array}$ & $\begin{array}{l}\text { Atech } \\
\text { Manufacturing }\end{array}$ & & $\mathrm{N} / \mathrm{A}$ \\
\hline Outlet barbs & $\begin{array}{l}\text { Sanitary White PVDF } \\
\text { Sngl-Barbed Tube } \\
\text { Fitting Adapter for } 1 / 4 " \\
\text { Tube ID X 1/4" NPT } \\
\text { Male Pipe }\end{array}$ & McMaster-Carr & www.mcmaster.com & $\begin{array}{l}5305 \\
5 \mathrm{~K} 21 \\
3\end{array}$ \\
\hline Hose Clamps & $\begin{array}{l}\text { Worm-Drive Hose \& Tu } \\
\text { Clamp with Thumb } \\
\text { Screw, 2-9/16" to } \\
\text { 3-1/2" Clamp Diameter } \\
\text { Range, 9/16" Band } \\
\text { Width, Packs of } 5 \\
\text { Th }\end{array}$ & AcMaster-Carr & www.mcmaster.com & $\begin{array}{l}5362 \\
\text { K24 }\end{array}$ \\
\hline $\begin{array}{l}\text { Female Luer- } \\
\text { Thread } \\
\text { Fitting } \\
\end{array}$ & $\begin{array}{l}\text { Female Luer x 10-32 } \\
\text { UNF thread, PP, 25/pk }\end{array}$ & Cole-Parmer & $\begin{array}{l}\text { www.coleparmer.co } \\
\text { m }\end{array}$ & $\begin{array}{l}4550 \\
0-60\end{array}$ \\
\hline Male Luer- & Male Luer Integral Lock & Value Plastic & www.valueplastic.co & XMT \\
\hline
\end{tabular}




\begin{tabular}{|c|c|c|c|c|}
\hline $\begin{array}{l}\text { Thread } \\
\text { Fitting }\end{array}$ & $\begin{array}{l}\text { Ring to 10-32 Special } \\
\text { Tapered Thread, White } \\
\text { Nylong }\end{array}$ & & $\mathrm{m}$ & LL-1 \\
\hline Qosina Barbs & $\begin{array}{l}\text { Female Luer Lock to } \\
\text { Barb Connector, Fits } \\
\text { 1/32" ID tubing }\end{array}$ & Qosina & www.qosina.com & $\begin{array}{l}1173 \\
3\end{array}$ \\
\hline $\begin{array}{l}\text { Male-to- } \\
\text { Male Fitting }\end{array}$ & $\begin{array}{l}\text { Male-to-Male Luer } \\
\text { Connector with One } \\
\text { Rotating Collar }\end{array}$ & Qosina & www.qosina.com & $\begin{array}{l}1766 \\
4\end{array}$ \\
\hline $\begin{array}{l}\text { Female-to- } \\
\text { Female } \\
\text { Coupler }\end{array}$ & $\begin{array}{l}\text { Female Luer Thread } \\
\text { Style Coupler }\end{array}$ & Value Plastics & $\begin{array}{l}\text { www.valueplastics.c } \\
\text { om }\end{array}$ & $\begin{array}{l}\text { FTLL } \\
\text { C-1 }\end{array}$ \\
\hline $\begin{array}{l}\text { Male-to-Barb } \\
\text { fitting }\end{array}$ & $\begin{array}{l}\text { Male Luer Integral Lock } \\
\text { Ring to } 200 \text { Series Barb, } \\
1 / 8 \text { " ( } 3.2 \mathrm{~mm}) \text { ID } \\
\text { Tubing, White Nylon }\end{array}$ & Value Plastics & $\begin{array}{l}\text { www.valueplastics.c } \\
\text { om }\end{array}$ & $\begin{array}{l}\text { MTL } \\
\text { L230- } \\
1\end{array}$ \\
\hline $\begin{array}{l}\text { Female Luer- } \\
\text { to-Barb }\end{array}$ & $\begin{array}{l}\text { Female Luer Thread } \\
\text { Style to } 200 \text { Series Barb, } \\
1 / 8 \text { " (3.2 mm) ID } \\
\text { Tubing, White Nylon }\end{array}$ & Value Plastics & $\begin{array}{l}\text { www.valueplastics.c } \\
\text { om }\end{array}$ & $\begin{array}{l}\text { FTLL } \\
230-1\end{array}$ \\
\hline Y-Connector & $\begin{array}{l}\text { Y-Connector with } 200 \\
\text { Series Barbs, } 1 / 8 \text { " }(3.2 \\
\text { mm) ID Tubing, White } \\
\text { Nylon }\end{array}$ & Value Plastics & $\begin{array}{l}\text { www.valueplatics.co } \\
\text { m }\end{array}$ & $\begin{array}{l}\text { Y230 } \\
-1\end{array}$ \\
\hline End Cap & $\begin{array}{l}\text { Male Luer Integral Lock } \\
\text { Ring Plug, Closed at } \\
\text { Grip, White Nylon }\end{array}$ & Value Plastics & $\begin{array}{l}\text { www.valueplastics.c } \\
\text { om }\end{array}$ & $\begin{array}{l}\text { LP4- } \\
1\end{array}$ \\
\hline $\begin{array}{l}\text { 3-Way Stop } \\
\text { Cock }\end{array}$ & $\begin{array}{l}\text { Three-Way Stopcock, } 2 \\
\text { Capped Female Luer } \\
\text { Thread Style \& Capped } \\
\text { Male Luer Lock, } \\
\text { Polycarbonate Body w/ } \\
\text { Polyethylene Handle }\end{array}$ & Value Plastics & $\begin{array}{l}\text { www.valueplastics.c } \\
\text { om }\end{array}$ & $\begin{array}{l}\text { VPB1 } \\
0000 \\
79 \mathrm{~N}\end{array}$ \\
\hline $\begin{array}{l}\text { 1-Way Stop } \\
\text { Cock }\end{array}$ & $\begin{array}{l}\text { One-Way Stopcock, } \\
\text { Capped Male Luer Lock } \\
\text { to Capped Female Luer } \\
\text { Thread Style, }\end{array}$ & Value Plastics & $\begin{array}{l}\text { www.valueplastics.c } \\
\text { om }\end{array}$ & $\begin{array}{l}\text { VP45 } \\
5980\end{array}$ \\
\hline
\end{tabular}




\begin{tabular}{|c|c|c|c|c|}
\hline & $\begin{array}{l}\text { Polycarbonate Body w/ } \\
\text { Polyethylene Handle }\end{array}$ & & & \\
\hline Tubing & $\begin{array}{l}\text { Tygon Silicone Tubing } \\
1 / 8 " \text { ID x } 1 / 4 " \text { OD }\end{array}$ & Cole Parmer & $\begin{array}{l}\text { www.coleparmer.co } \\
\text { m }\end{array}$ & $\begin{array}{l}\text { WU- } \\
9570 \\
2-06\end{array}$ \\
\hline $\begin{array}{l}\text { Gas } \\
\text { Permeable } \\
\text { Tubing }\end{array}$ & $\begin{array}{l}\text { Tygon Silicone Tubing } \\
33501 / 8 \times 3 / 16\end{array}$ & Cole Parmer & $\begin{array}{l}\text { www.coleparmer.co } \\
\text { m }\end{array}$ & $\begin{array}{l}\text { EW- } \\
9570 \\
2-05\end{array}$ \\
\hline $\begin{array}{l}50 \mathrm{ml} \\
\text { Conical }\end{array}$ & $\begin{array}{l}\text { BD FalconTM } 50 \mathrm{ml} \\
\text { Conical-Bottom } \\
\text { Polypropylene Tube }\end{array}$ & $\mathrm{BD}$ & www.bd.com & $\begin{array}{l}3520 \\
98\end{array}$ \\
\hline Loctite 4011 & $\begin{array}{l}\text { Prism } 4011 \text { Medical } \\
\text { Glue, } 20 \mathrm{Gm} \text { Net } \\
\text { Weight Bottle }\end{array}$ & McMaster-Carr & www.mcmaster.com & $\begin{array}{l}1818 \\
\mathrm{~A} 4\end{array}$ \\
\hline $\begin{array}{l}\text { Loctite } \\
\text { Solvent }\end{array}$ & $\begin{array}{l}\text { LOCTITE } 768 \text { X-NMS } \\
\text { Clean Up Solvent For } \\
\text { Instant Adhesive } 52 \mathrm{ml}\end{array}$ & Ellsworth & www.ellsworth.com & $\begin{array}{l}7682 \\
0\end{array}$ \\
\hline
\end{tabular}



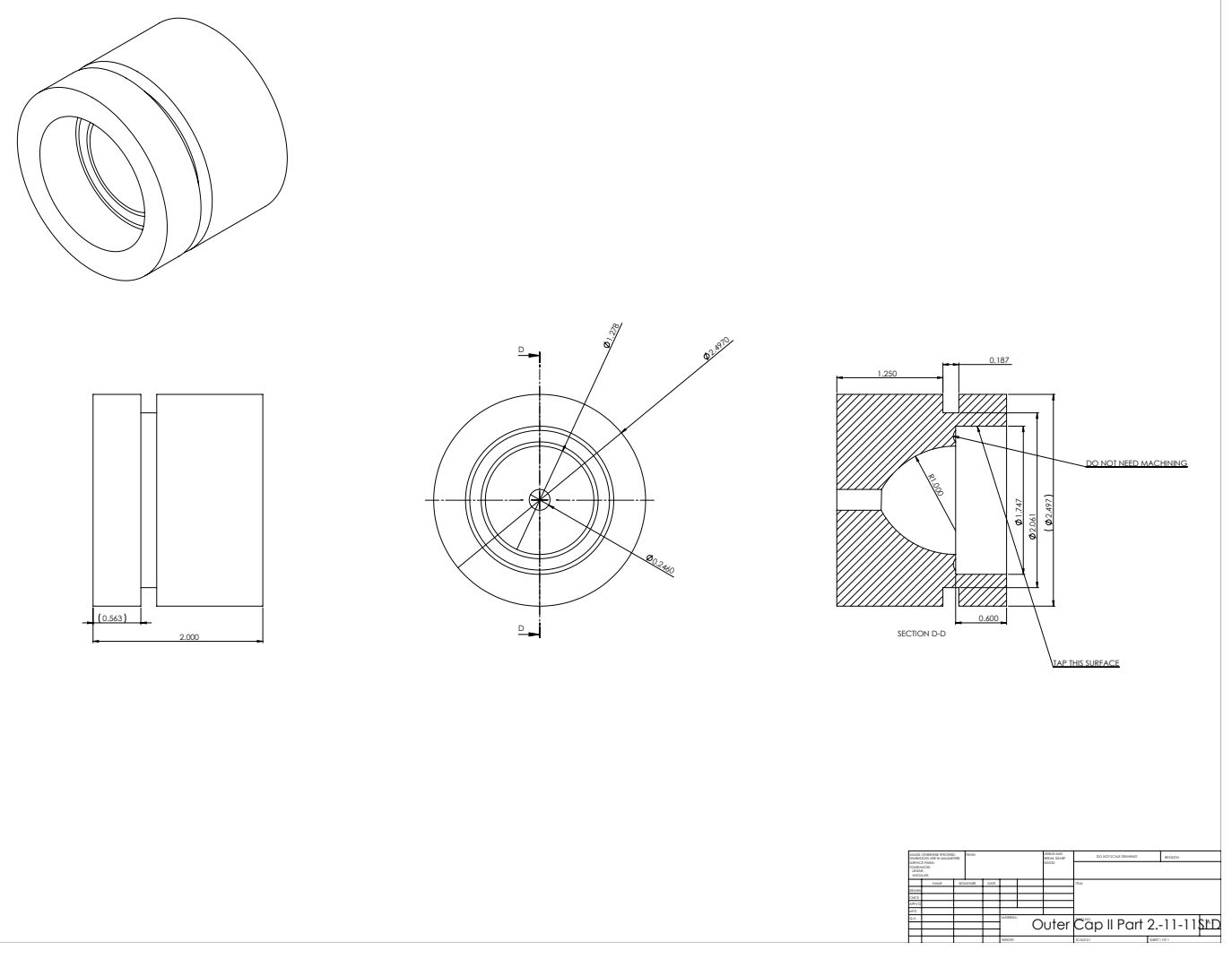

NOTE: MAKE 2
OF THESE
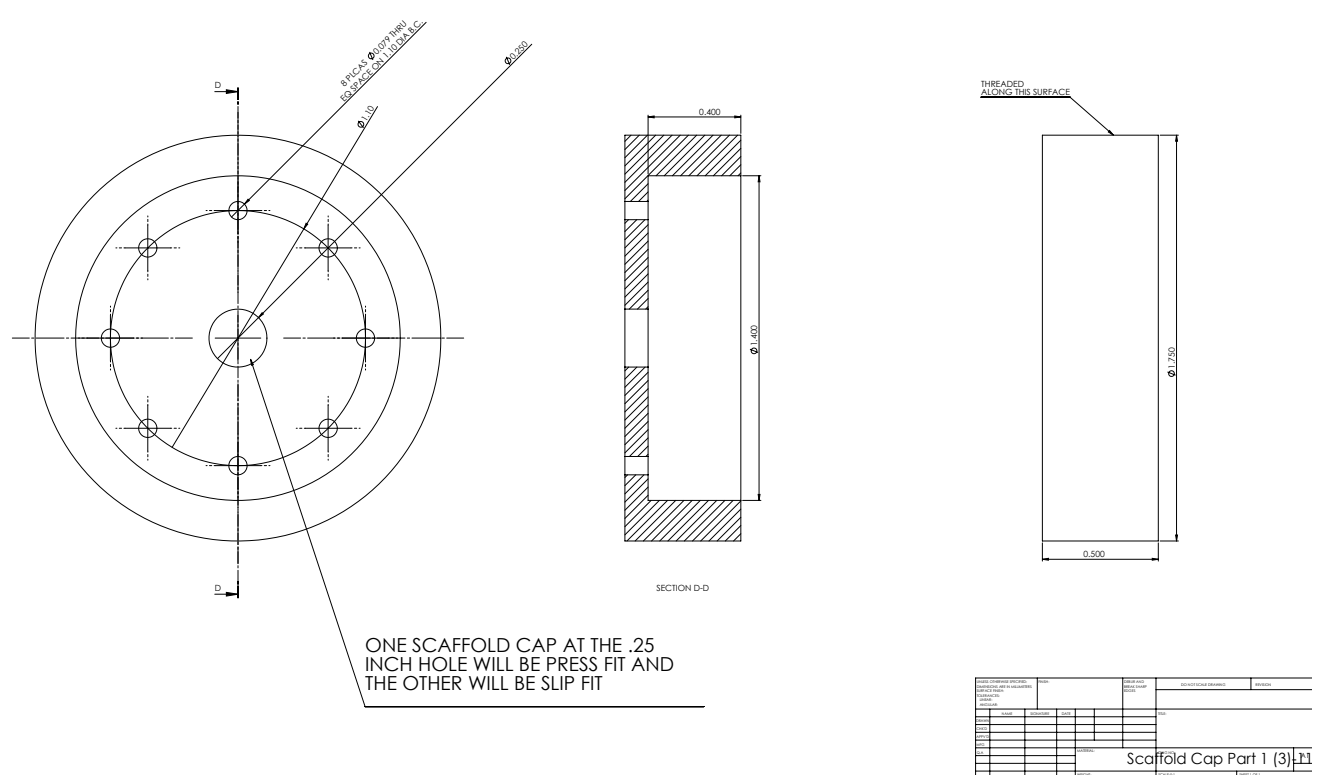


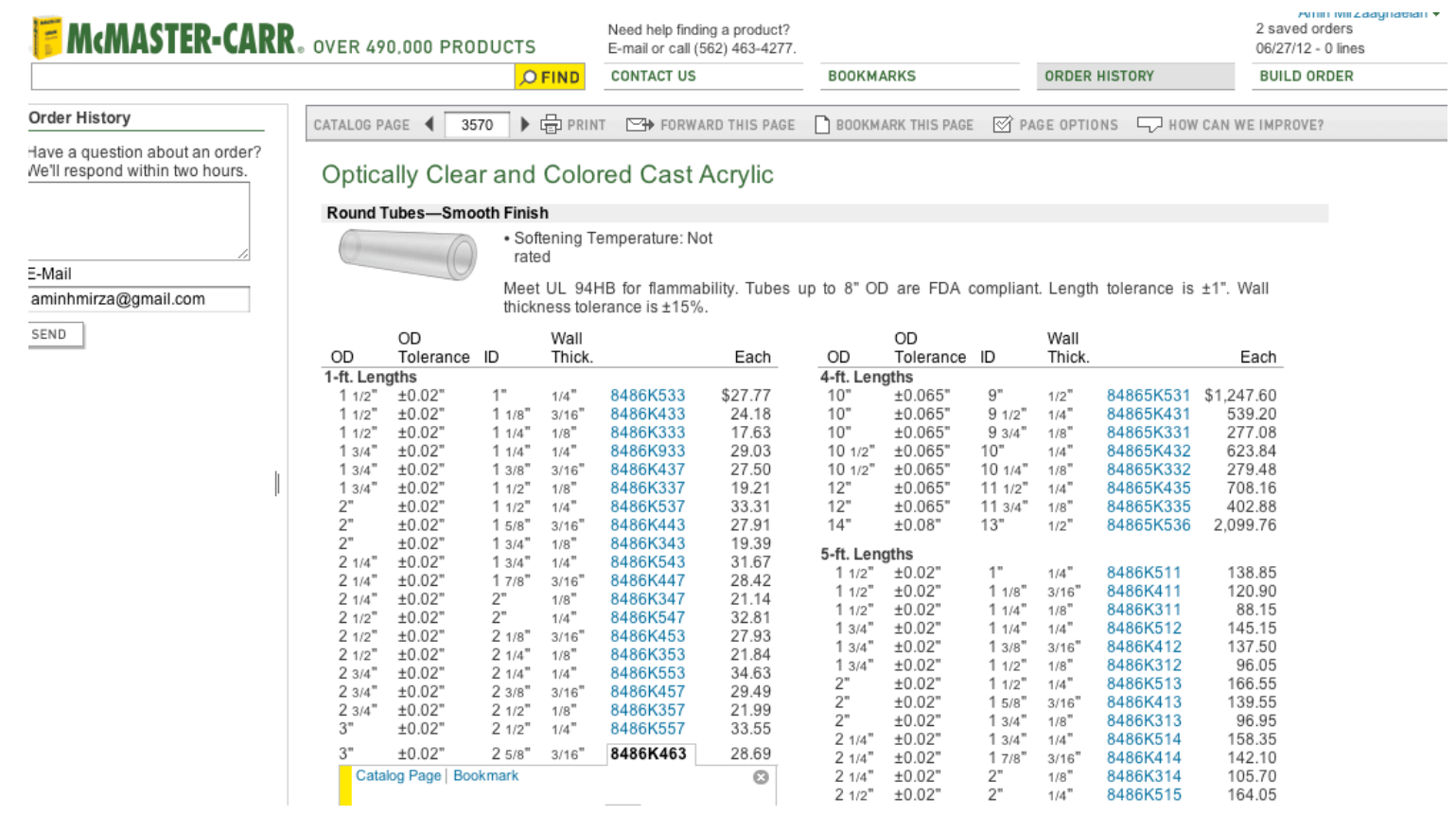

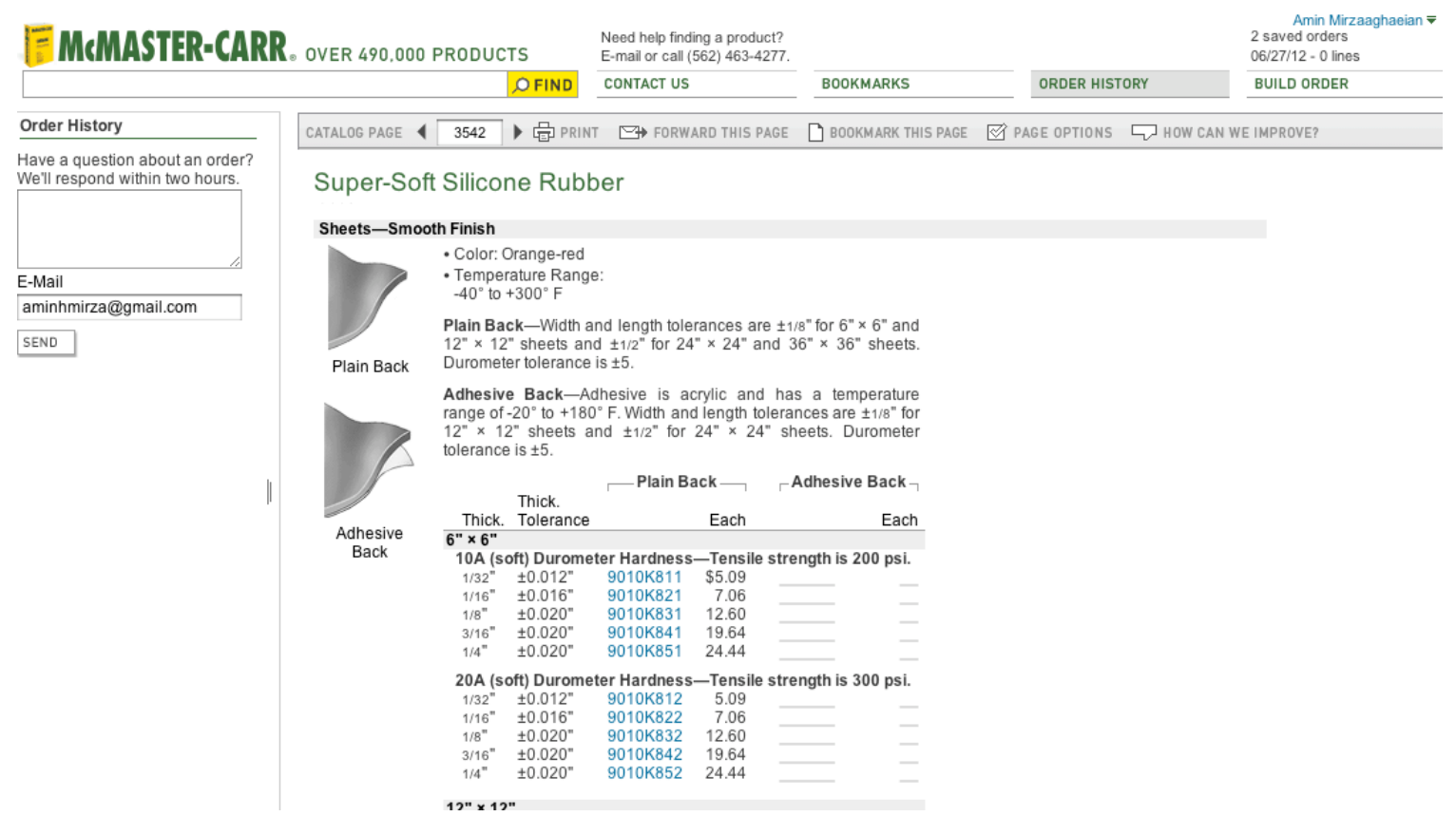




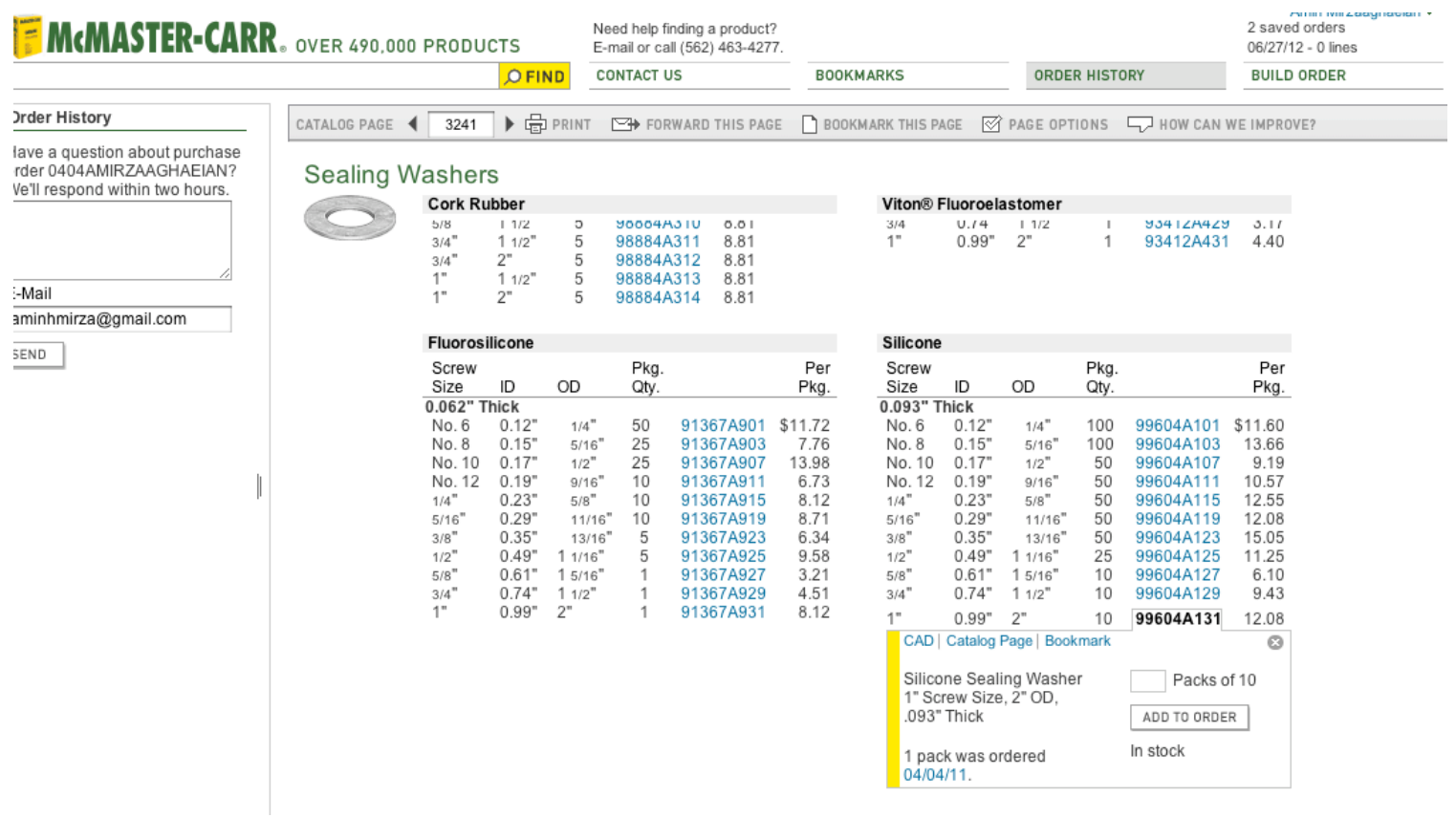

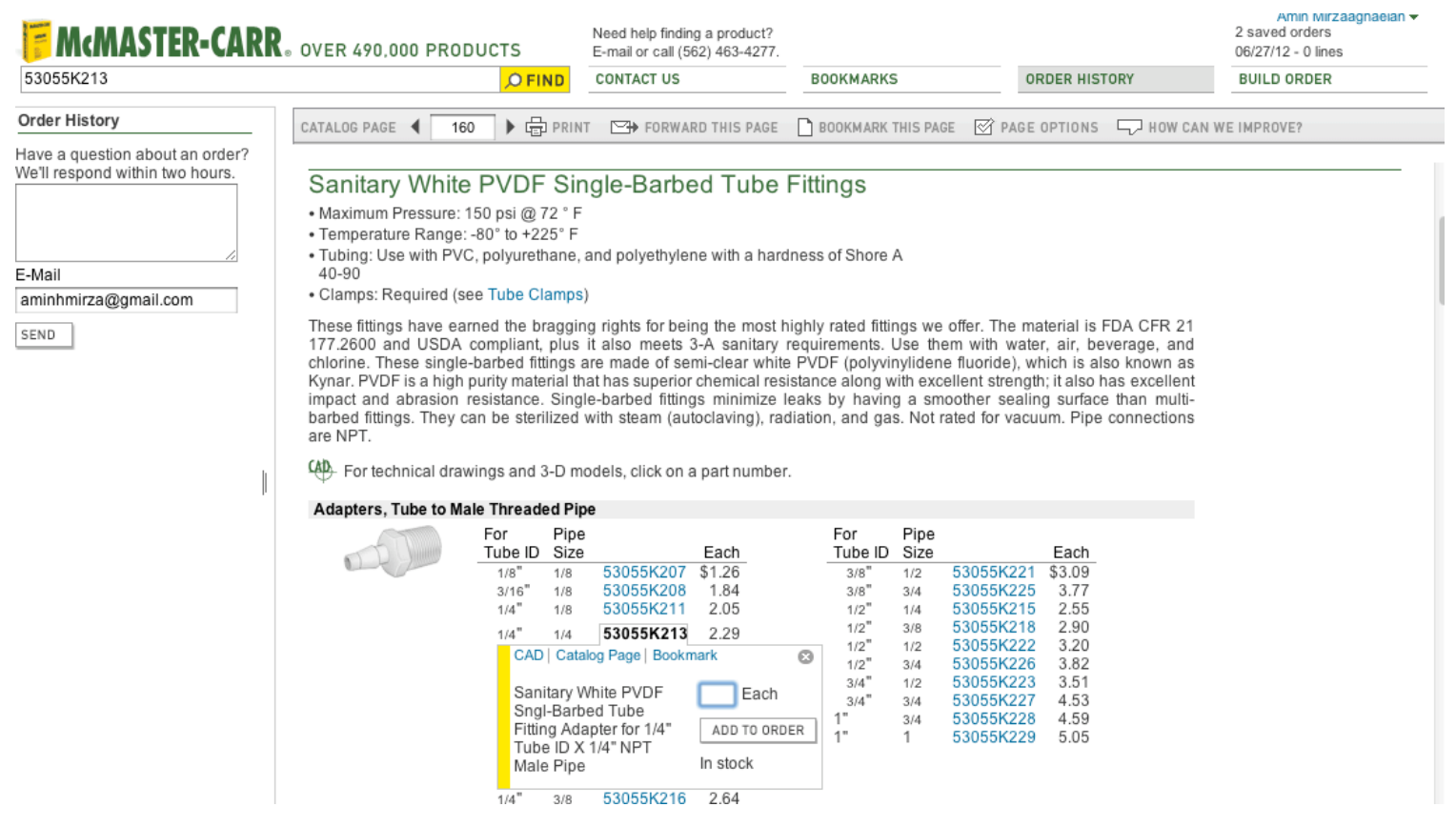




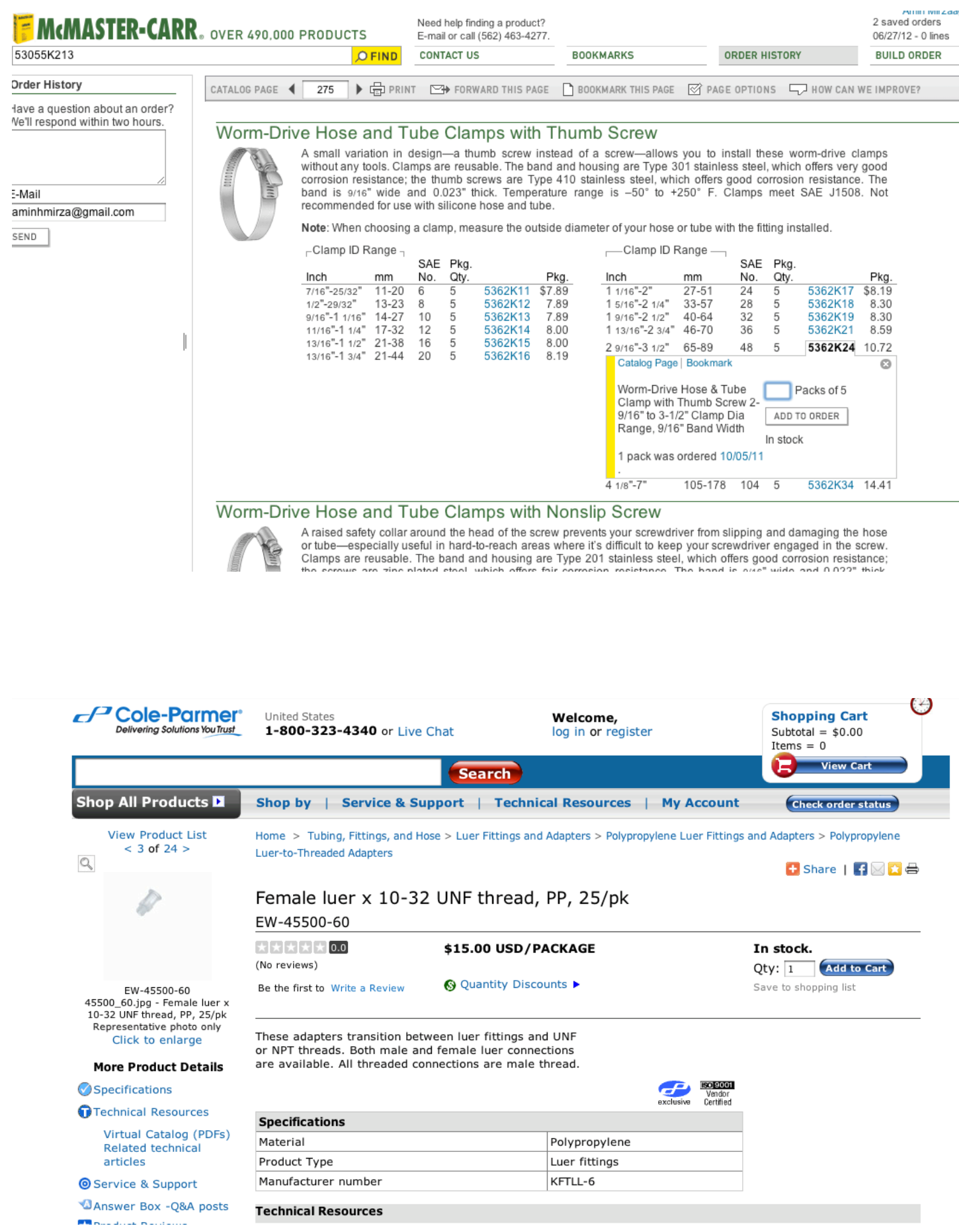



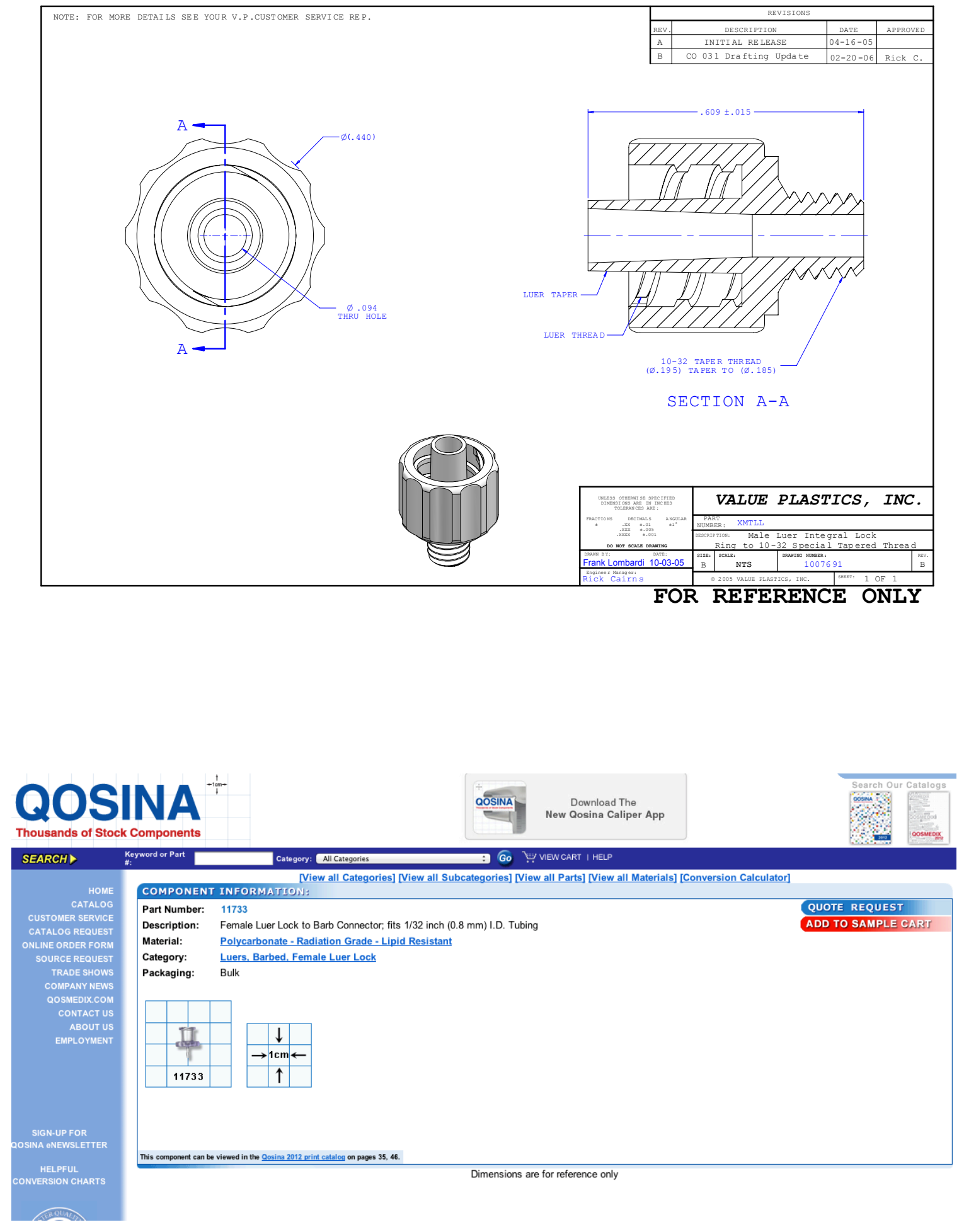


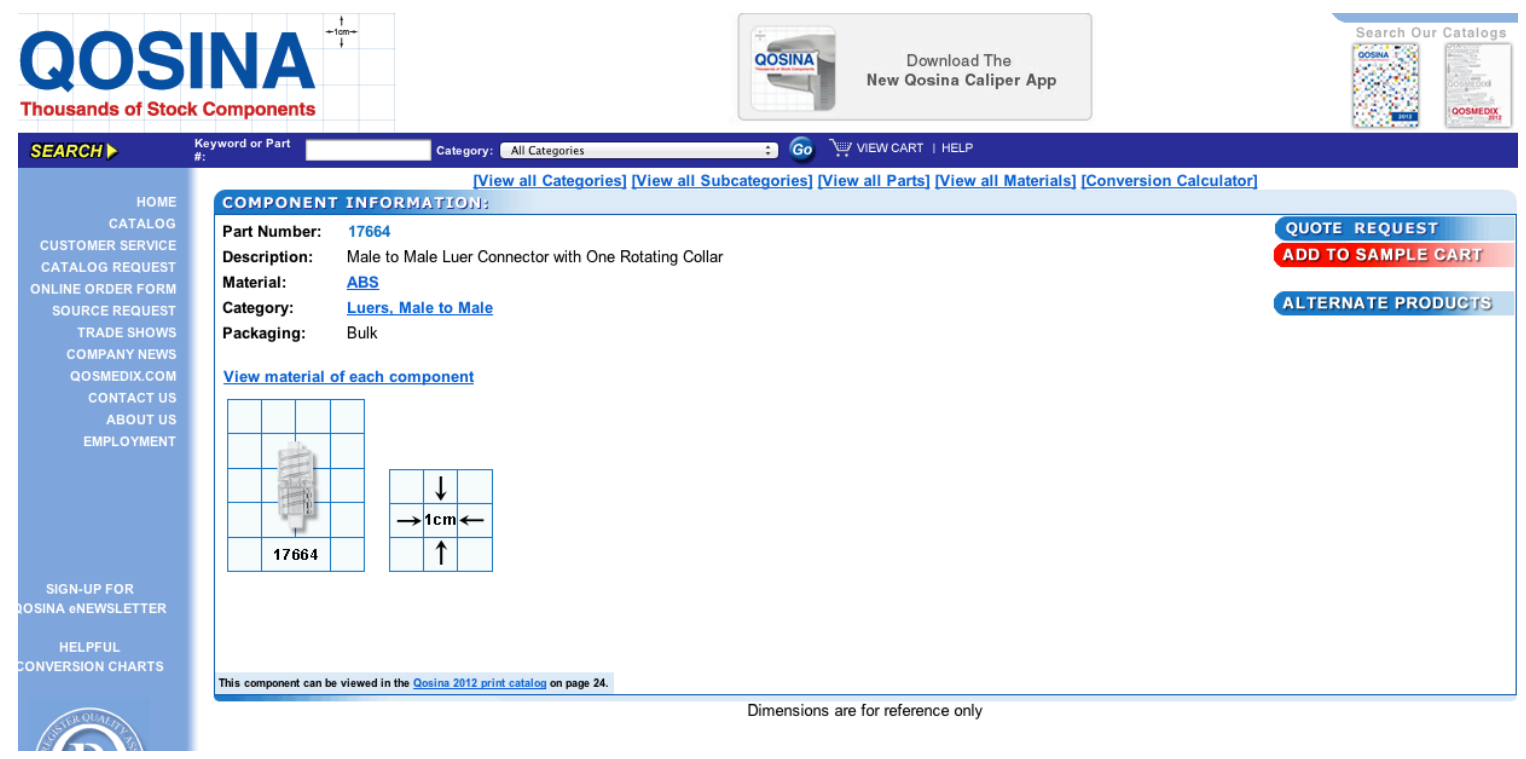



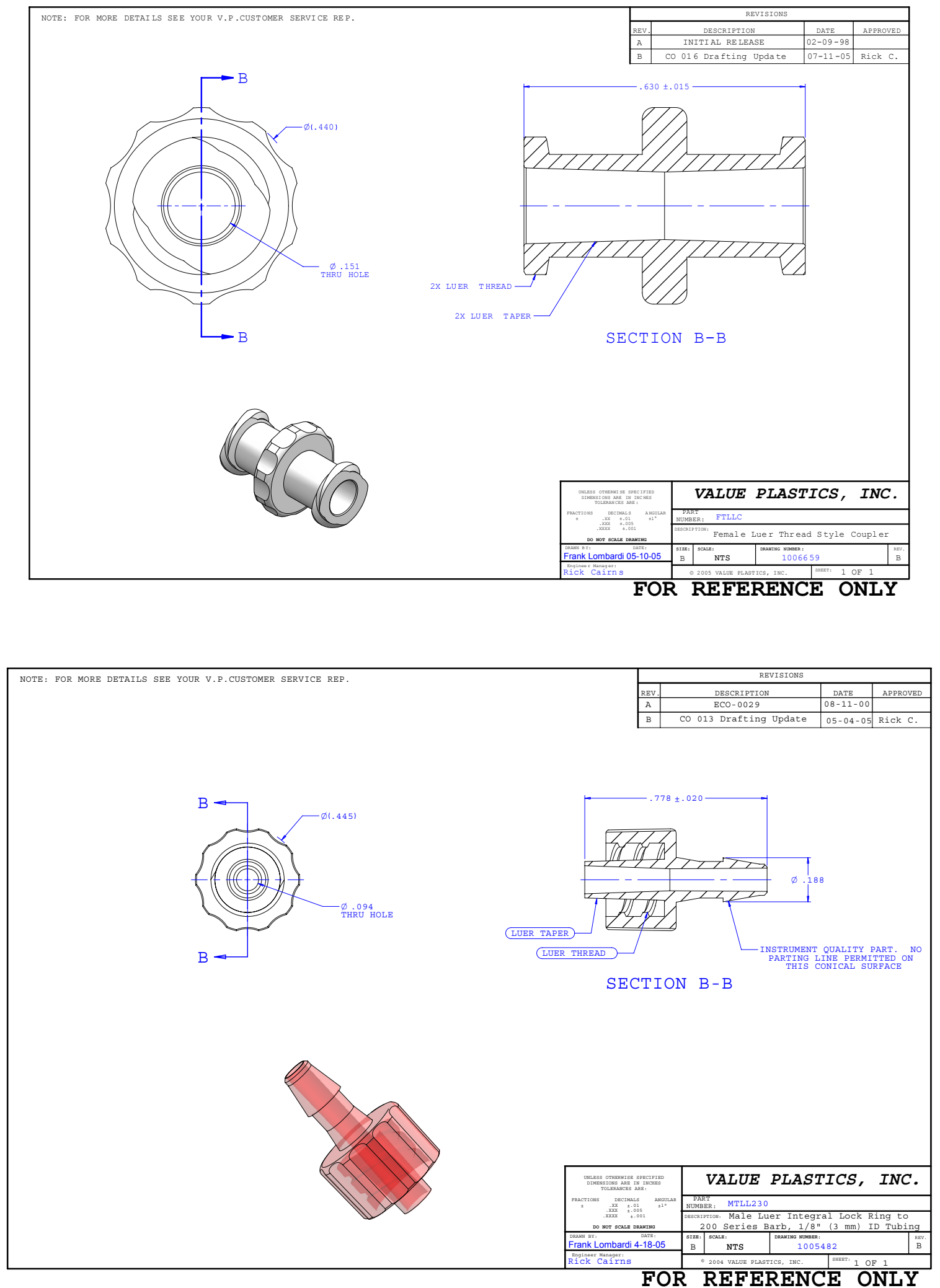


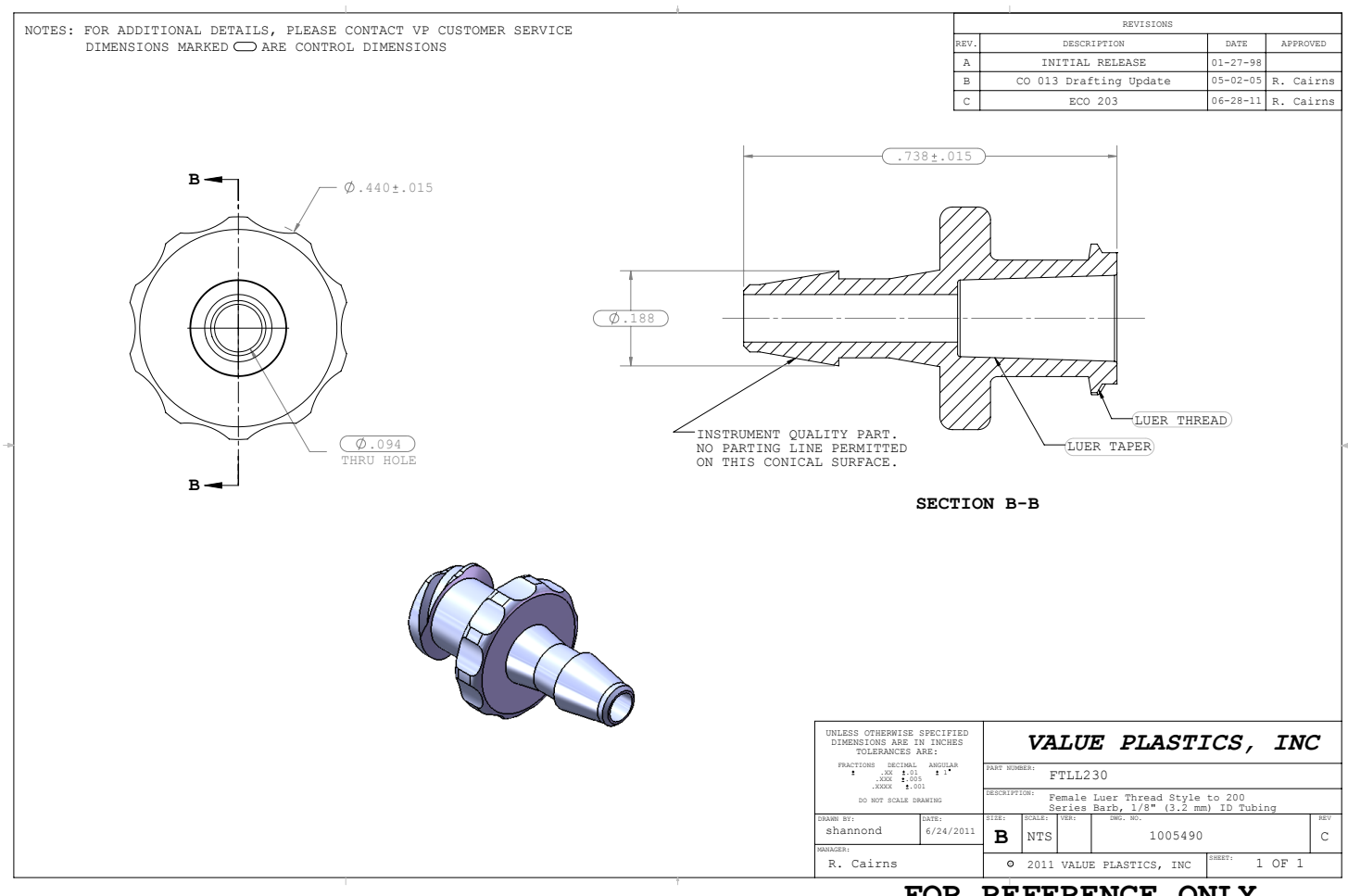

FOR REFERENCE ONLY

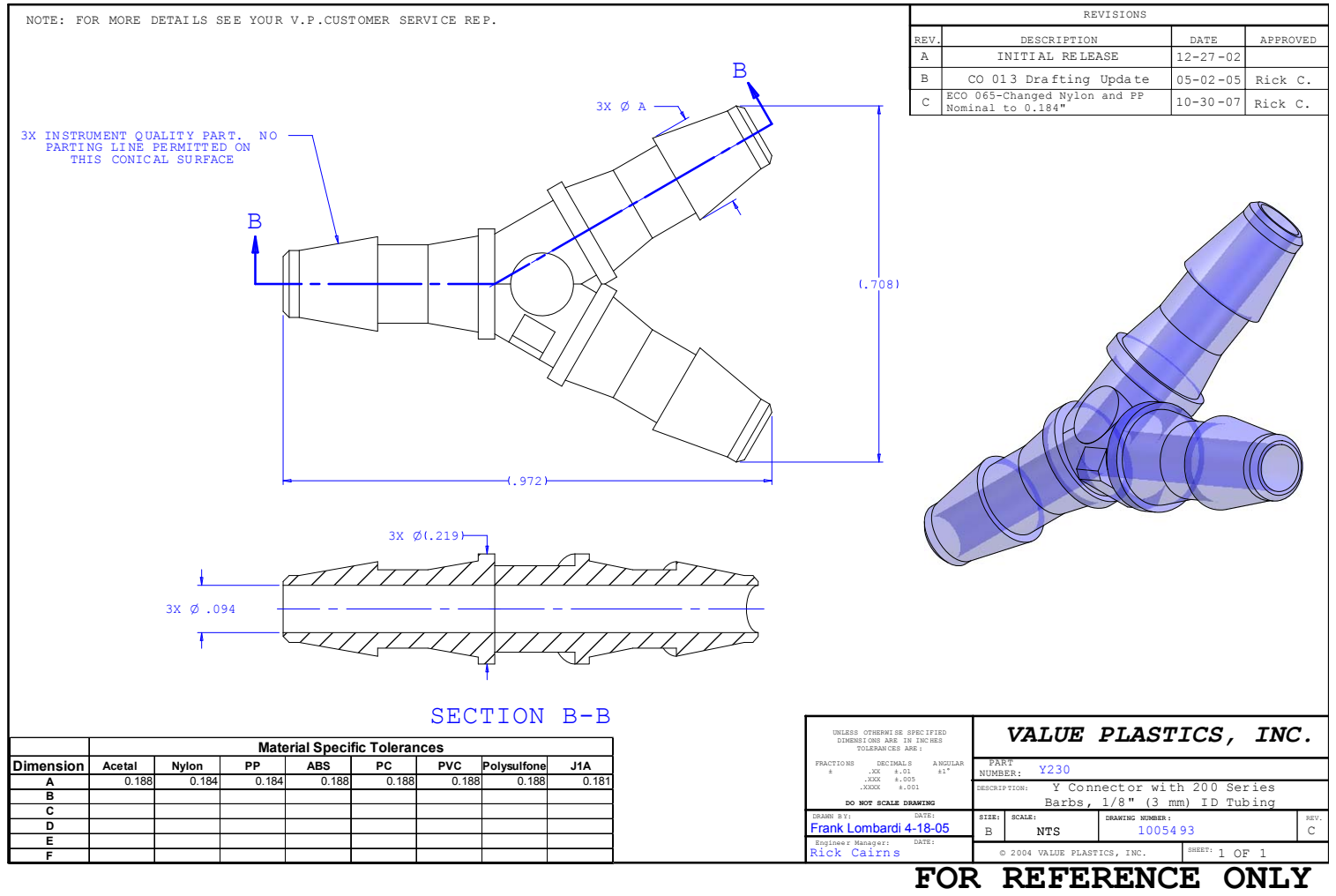




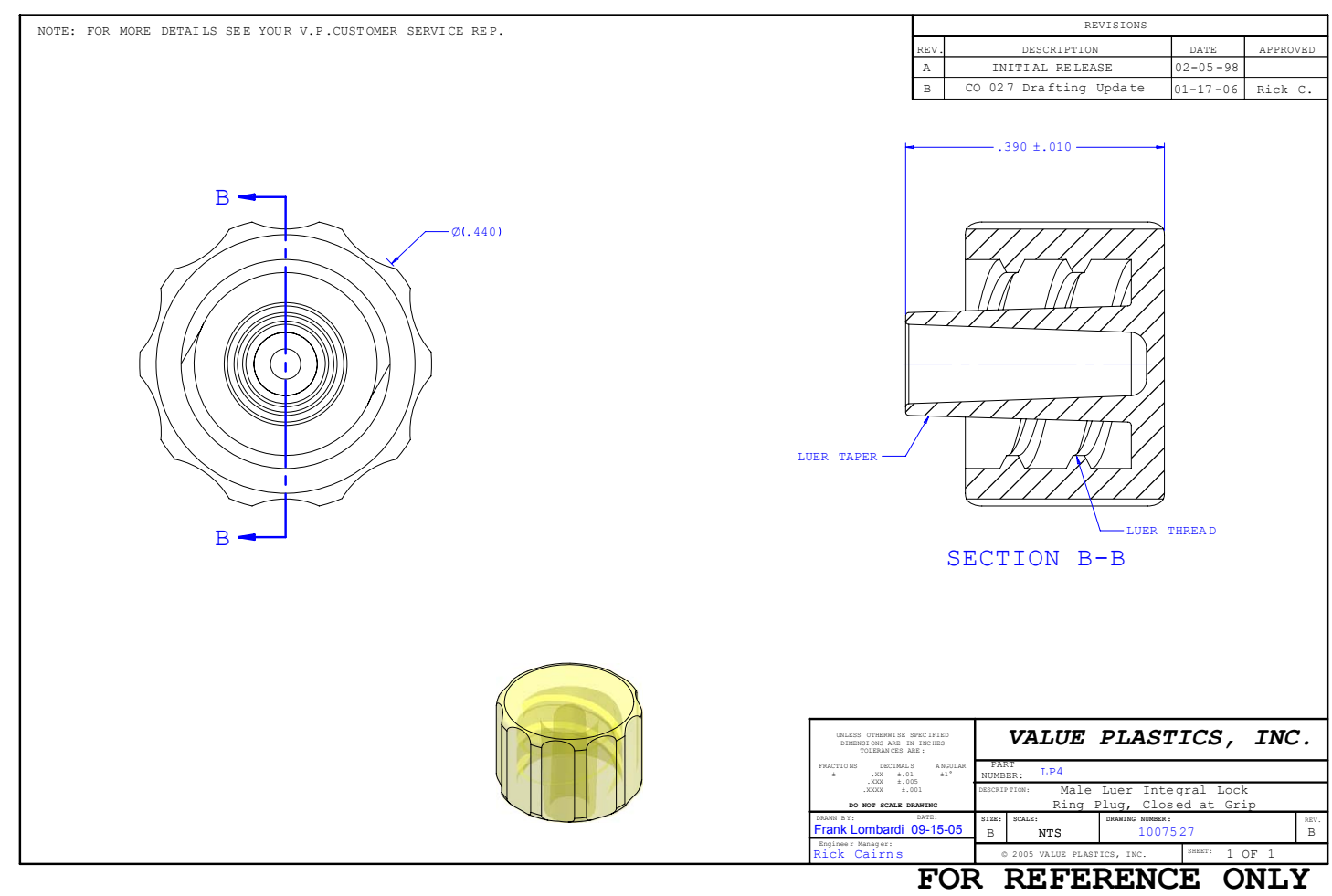




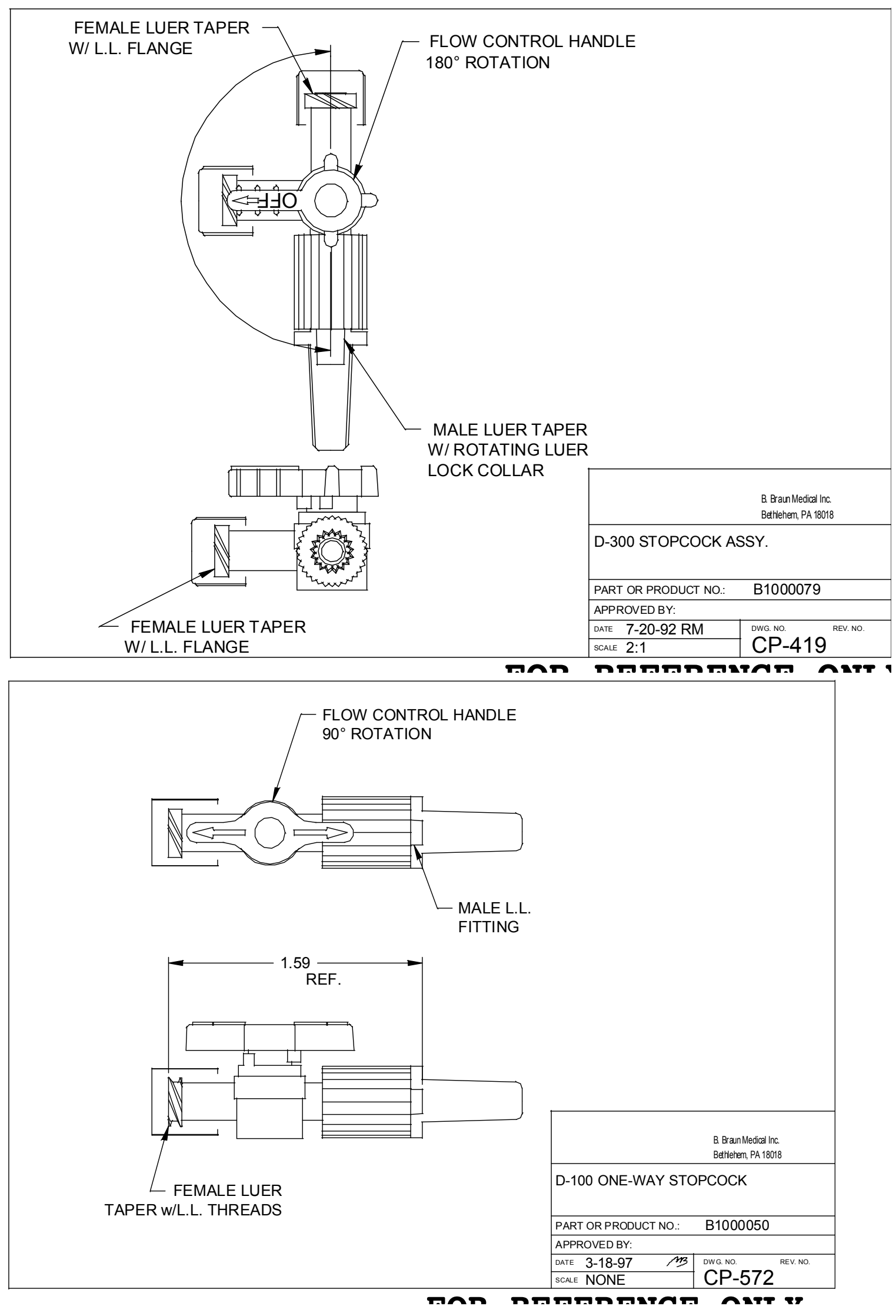




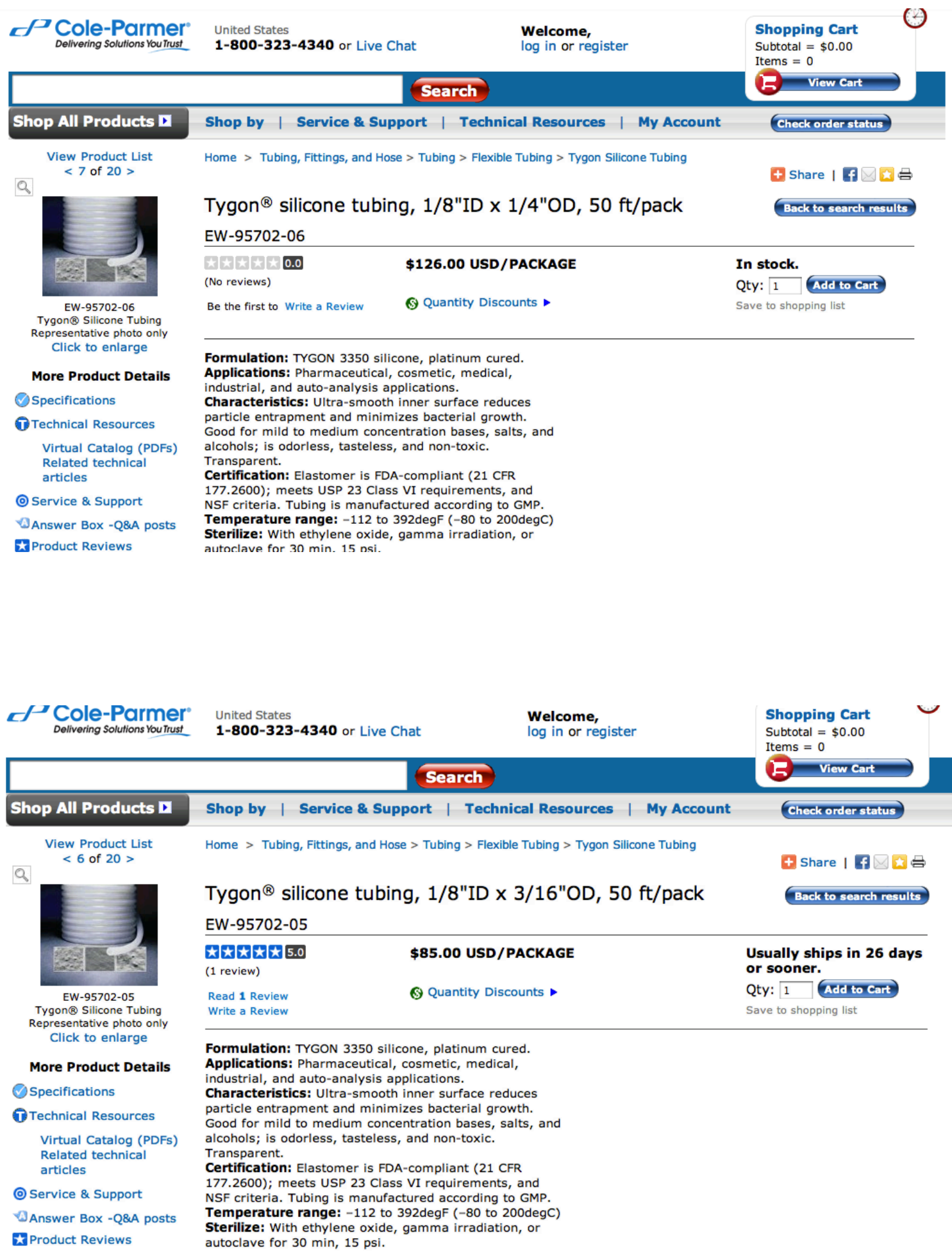


BD

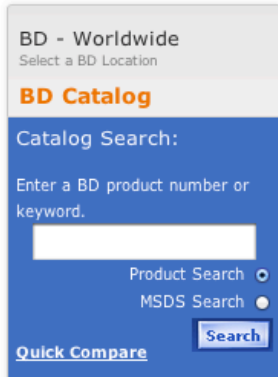

- How to Order

- MSDS

- Quality Certificates

- Catalog Feedback

- BD Catalog Home

Other Catalogs:

- BD Biosciences Catalog

About BD | Contact BD | BD Worlawide | Careers | Products | Investors | News \& Medla | Privacy | Terms \& Conditions

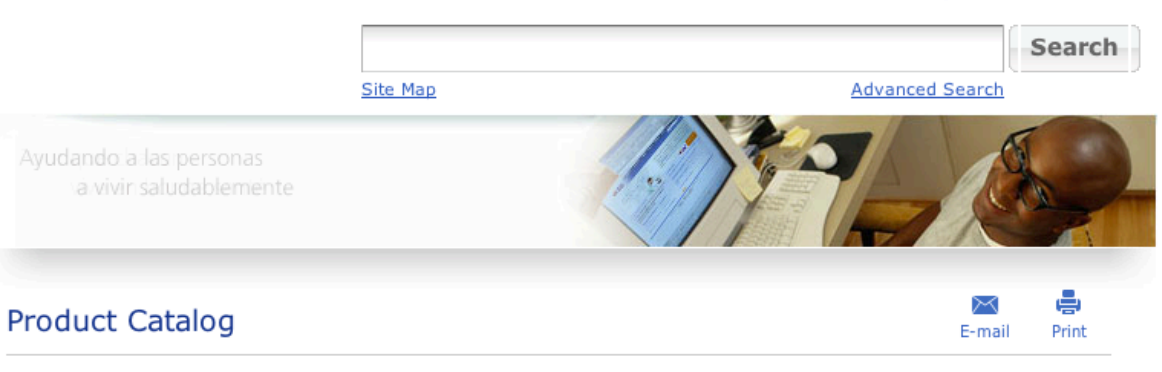

Welcome to the BD (Becton, Dickinson and Company) Catalog of medical supplies, devices, laboratory equipment and diagnostic products.

Centrifuge and Test Tubes

Product Number: 352098

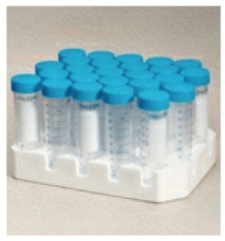

Safety Data Sheet

More information

Certificate of Analysis

352098 - $50 \mathrm{ml}$ high-clarity polypropylene conical centrifuge tube, $16000 \mathrm{RCF}$ rating. Sterile. (25/sp, 500/ca)

Approximate tube dimensions are $30 \mathrm{~mm}$ O.D., $115 \mathrm{~mm}$ length.

Dark blue printed graduations, white writing patch.

Polyethylene dome-seal screw cap offers positive seal over the full circumference.

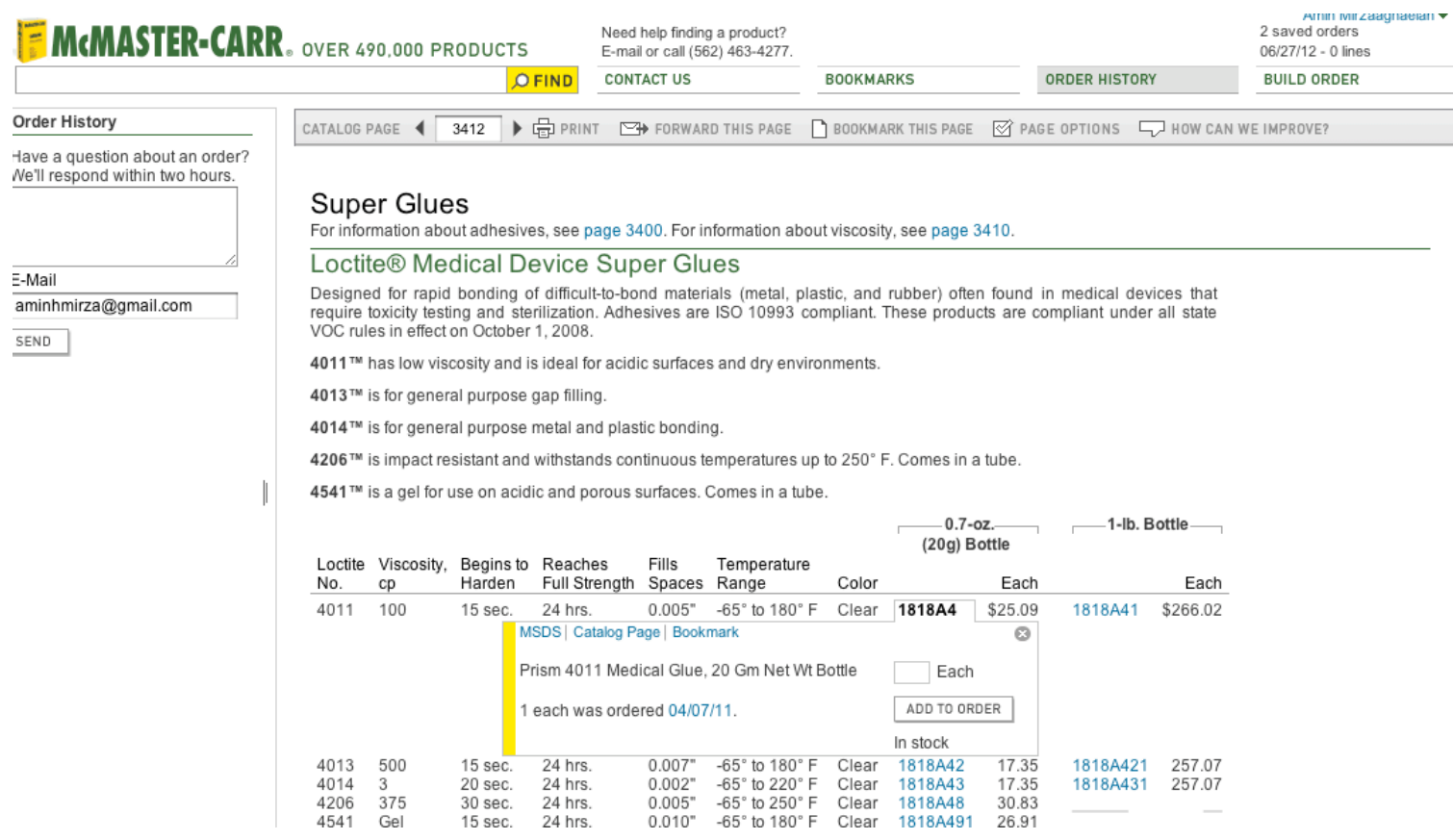




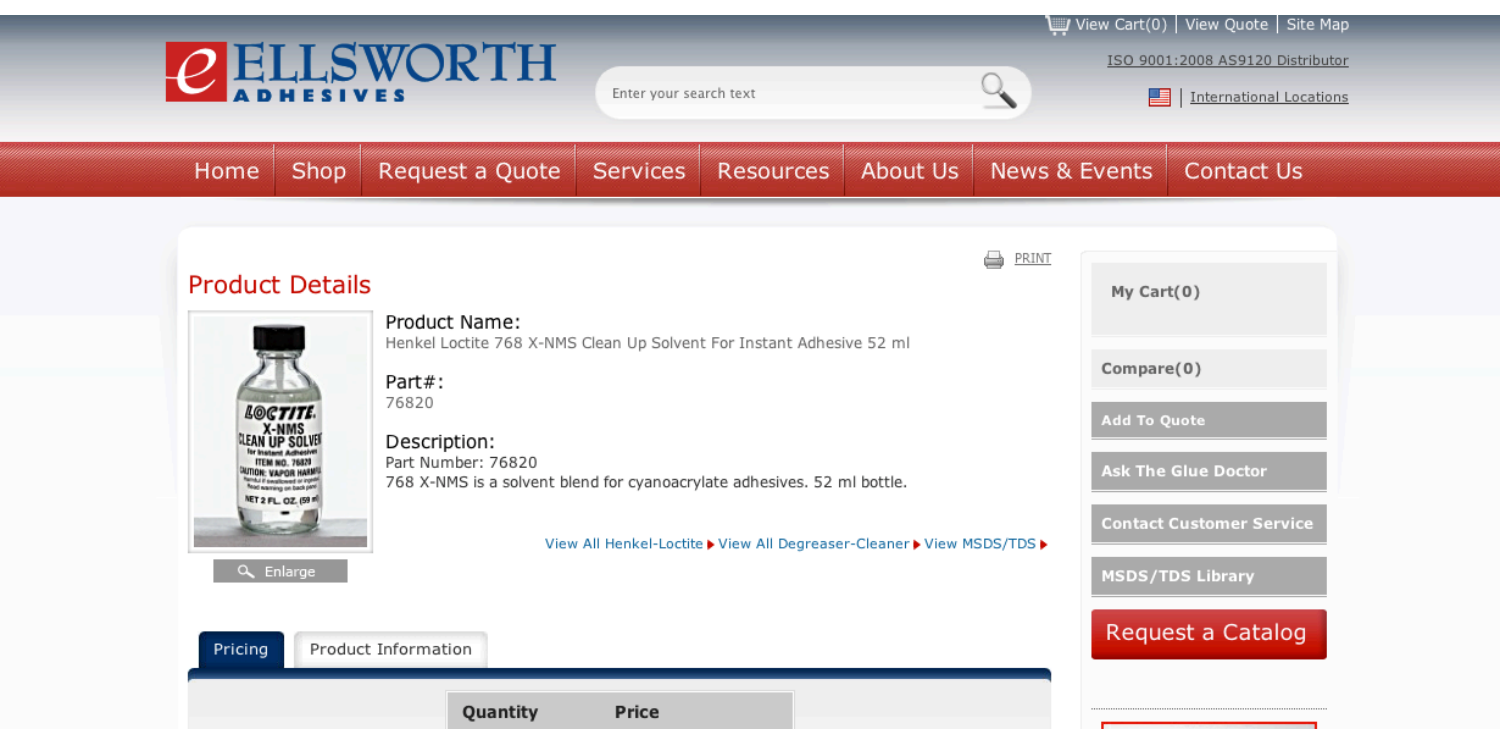




\section{Appendix C - Barb Testing}

Barb testing was the first cellular based assessment performed using the barbs used in BBB bioreactor. The barb testing investigated whether the smaller scale barb and scaffold installation technique functioned properly. In addition, the barbs were also evaluated for compatibility in the Tupperware bioreactors. The Tupperware bioreactors were used prior to the BBB bioreactor, since validation that the barb-scaffold-barb configuration worked in an existing bioreactor system would help explain the BBB bioreactor's efficiency. Using the non-sterile set-up protocol, a single $3.5 \mathrm{~cm}$ scaffold sutured to Qosina barbs was fitted into the bioreactor secured by rotating male luer locks. Then bioreactor media was poured into the chamber, while conditioning media was inserted into the reservoir. Once primed, the appropriate pump setting was selected and transmural flow conditions were operated for twenty-four hours. Transmural flow allowed radial dispersion of fluid away from the scaffold and out through the transmural outlet. These proteins would later facilitate the growth of cellular components within the scaffold. After twenty-four hours of conditioning, fibroblasts conditioned in one T75 flask up to $90-100 \%$ confluency was obtained and injected into the cell induction port. Once the cells were in the system, transmural flow was continued to allow the cells to be pushed radially onto the inner lumen of the scaffold for one hour. After an hour, flow was switched to transluminal flow for the remainder of the 24-hour experiment. The large incubator was set to $5 \% \mathrm{CO}_{2}$ and $37^{\circ} \mathrm{C}$, replicating the physiological environment of the human body. 
The barb testing experiments were assessed using BBI and Scanning Electron

Microscope (SEM) images. These images were used for qualitative evaluation in a short proof-of-concept study. BBI images display fibroblast nuclei fluorescing and distribution of cells throughout regions of the scaffold. While SEM images enable us to identify cell coverage on the inner lumen of the scaffold, along with the perception of the depth.
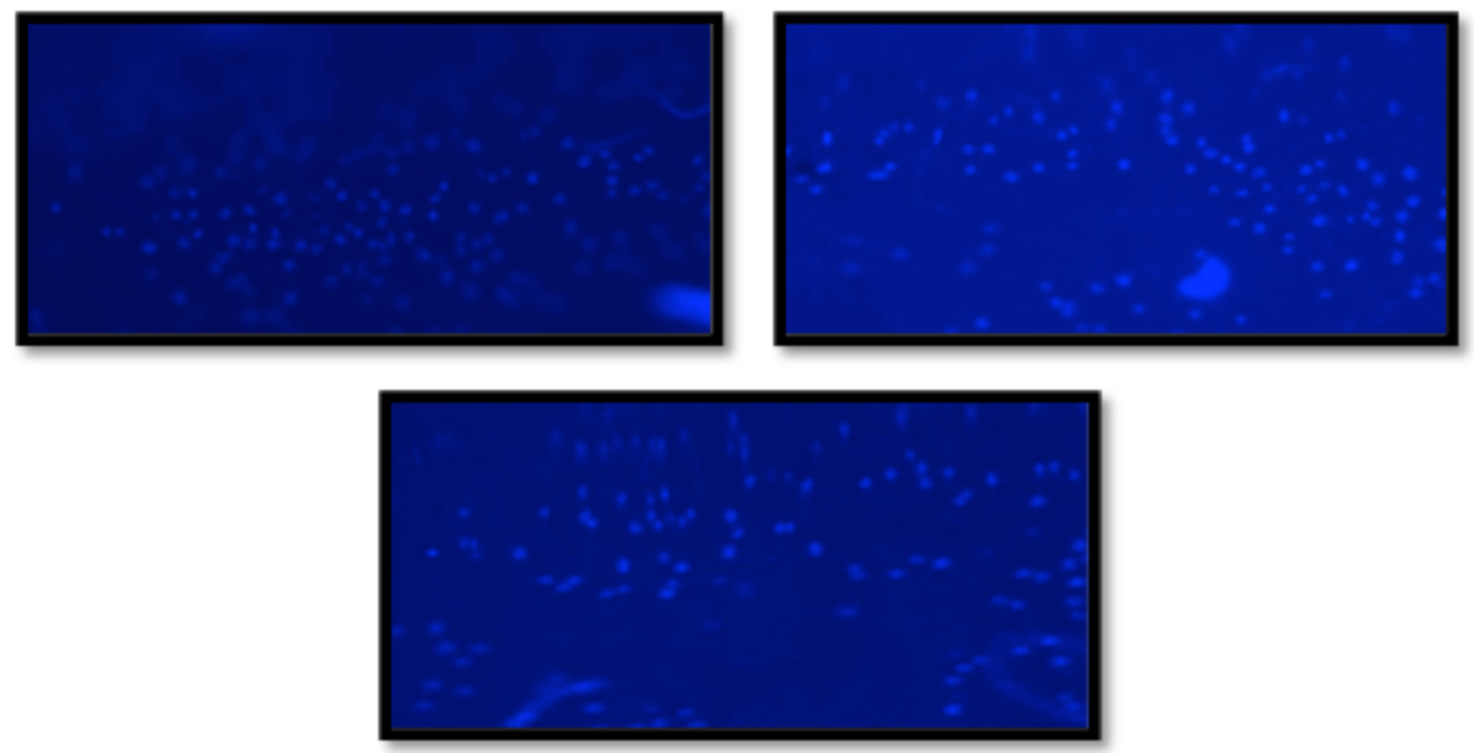

BBI images showing presence of cells on scaffold proximally (top left), middle (top right), and distally (bottom center). 


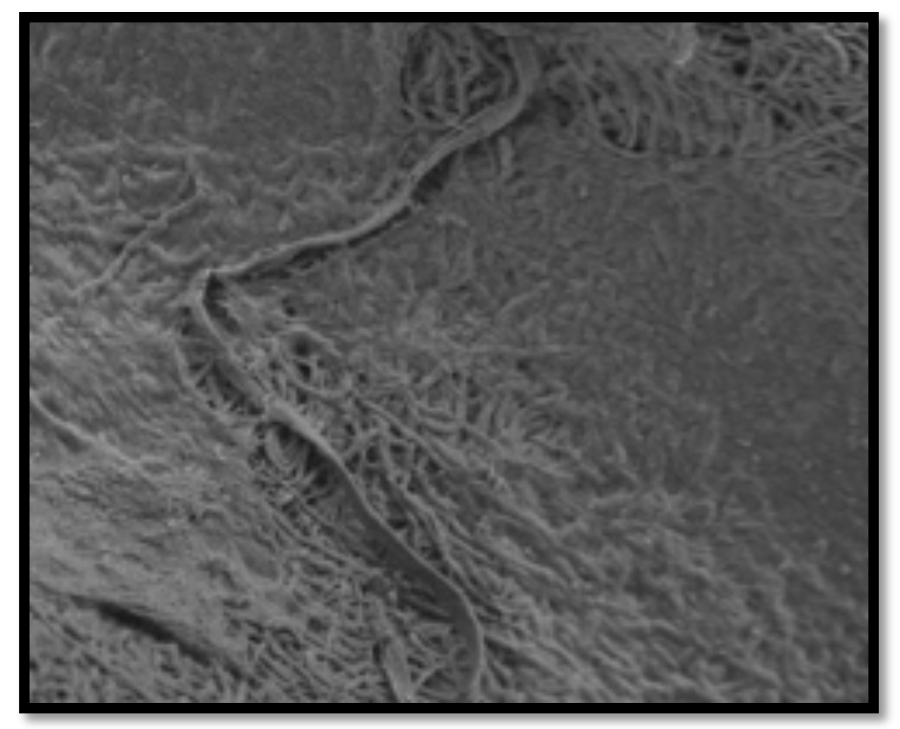

SEM Image of scaffold lumen imaged at 180X magnification. Designation between cell coverage and bare scaffold can be seen due to blade scrape when cutting the scaffold.

Results from barb testing showed that the barb component of the bioreactor functioned in the Tupperware bioreactors regularly used in the lab. Accordingly, the barbs were expected to function effectively in the BBB bioreactor. Barb testing provided qualitative information that demonstrated good distribution of cells across all regions of the scaffold. The positive results from barb testing were translated to single-scaffold experiments in the BBB bioreactor; again displaying the presence of cells across proximal, middle, and distal regions of the scaffold. This showed that the BBB bioreactor was able to produce similar results to the Tupperware bioreactor used in the lab, matching performance and ease of use. These proof-of-concept studies served as a preliminary baseline for the multiscaffold experiments that would test the true function of the designed bioreactor and its ability for long-term culture. 


\section{Appendix D - Protocols}

\section{Assembly Protocol}

\begin{tabular}{|c|c|c|c|}
\hline Component & Company & Part Number & Quantity \\
\hline Outer Housing & McMaster-Carr & $8486 \mathrm{~K} 463$ & 1 \\
\hline Outer Caps & $\begin{array}{l}\text { Atech } \\
\text { Manufacturing }\end{array}$ & N/A & 2 \\
\hline Bioreactor Cap & McMaster-Carr & $8486 \mathrm{~K} 473$ & 1 \\
\hline Internal O-ring & McMaster-Carr & 99604A131 & 2 \\
\hline $\begin{array}{l}\text { Custom Square } \\
\text { O-ring }\end{array}$ & McMaster-Carr & $9010 \mathrm{~K} 842$ & 2 \\
\hline Scaffold Cap & $\begin{array}{l}\text { Atech } \\
\text { Manufacturing }\end{array}$ & N/A & 2 \\
\hline Outlet barbs & McMaster-Carr & $53055 \mathrm{~K} 213$ & 2 \\
\hline Hose Clamps & McMaster-Carr & $5362 \mathrm{~K} 24$ & 3 \\
\hline $\begin{array}{l}\text { Female Luer- } \\
\text { Thread Fitting }\end{array}$ & Cole-Parmer & $45500-60$ & 4 \\
\hline $\begin{array}{l}\text { Male Luer- } \\
\text { Thread Fitting }\end{array}$ & Value Plastic & XMTLL-1 & 4 \\
\hline Qosina Barbs & Qosina & 11733 & 8 \\
\hline $\begin{array}{l}\text { Male-to-Male } \\
\text { Fitting }\end{array}$ & Qosina & 17664 & 4 \\
\hline $\begin{array}{l}\text { Female-to- } \\
\text { Female Coupler }\end{array}$ & Value Plastics & FTLLC-1 & 1 \\
\hline $\begin{array}{l}\text { Male-to-Barb } \\
\text { fitting }\end{array}$ & Value Plastics & MTLL230-1 & 6 \\
\hline $\begin{array}{l}\text { Female Luer-to- } \\
\text { Barb }\end{array}$ & Value Plastics & FTLL230-1 & 4 \\
\hline Y-Connector & Value Plastics & Y230-1 & 1 \\
\hline End Cap & Value Plastics & LP4-1 & 2 \\
\hline $\begin{array}{l}\text { 3-Way Stop } \\
\text { Cock }\end{array}$ & Value Plastics & VPB1000079N & 1 \\
\hline $\begin{array}{l}\text { 1-Way Stop } \\
\text { Cock }\end{array}$ & Value Plastics & VP455980 & 2 \\
\hline Tubing & Cole Parmer & WU-95702-06 & 5 \\
\hline $\begin{array}{l}\text { Gas Permeable } \\
\text { Tubing }\end{array}$ & Cole Parmer & EW-95702-05 & 2 \\
\hline $50 \mathrm{ml}$ Conical & BD & 352098 & 1 \\
\hline
\end{tabular}


1. Custom O-ring: Place custom square O-ring into O-ring groove located on the outer caps. Be sure not to

excessively compress O-ring into groove, allow it to sit to allow the O-ring to uniformly wrap around the outer cap. Excessive compression will allow risk of leaking from the ends of the bioreactor

2.Scaffold Cap 1: Based on the number of scaffolds being used in the experiment, connect luer to thread fittings onto scaffold cap. Then connect male-to-male couplings to scaffold cap.
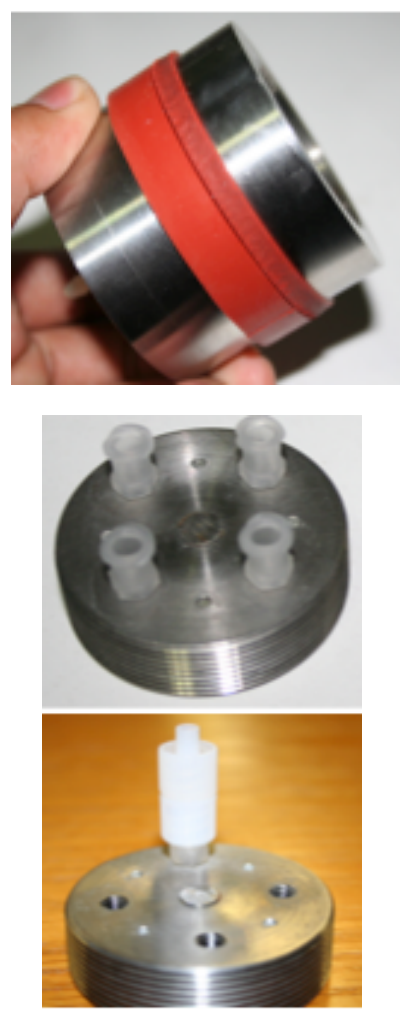

3. Scaffold Cap 2: Connect Value Plastic fittings.

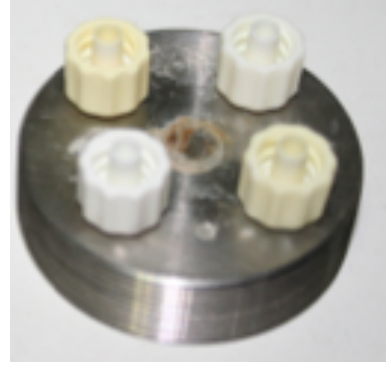

4. Place internal O-rings into outer cap.

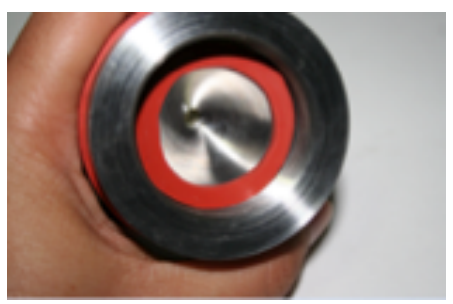

5. Thread scaffold caps to outer caps.

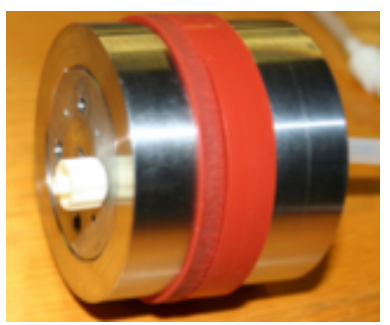


6. Scaffold Installation: (Refer to Scaffold Assembly Protocol for instructions) Take scaffold and attach to stationary end.

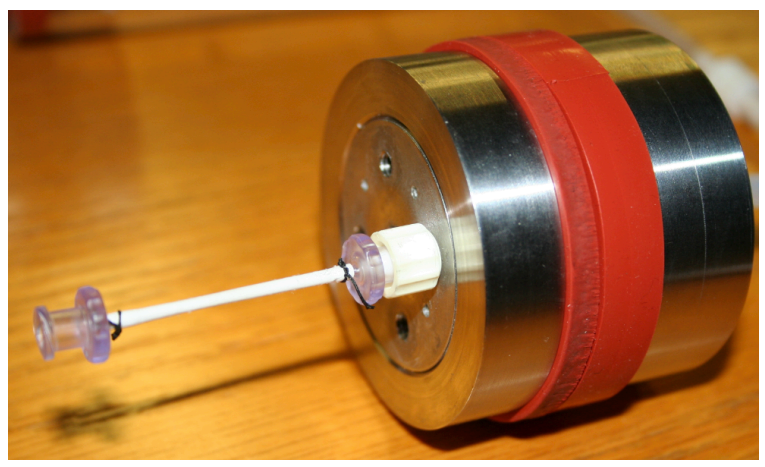

7. Push both outer caps into outer housing. Make sure to leave at least $1 / 8$ " of silicon gasket material hanging out of the outer housing.

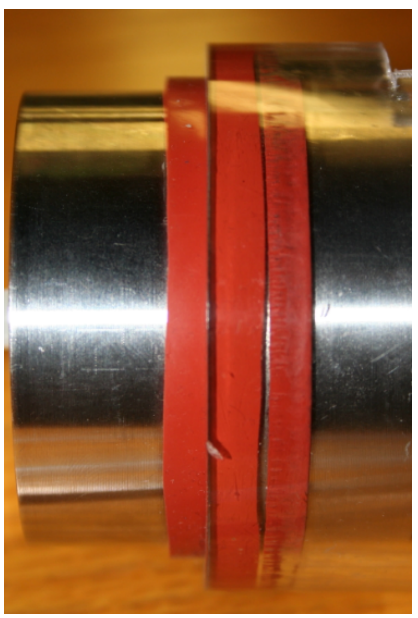

8. Connect the other end of the scaffold to the fittings on the other outer cap. Make sure that the scaffolds are secured in the male-to-male fitting.

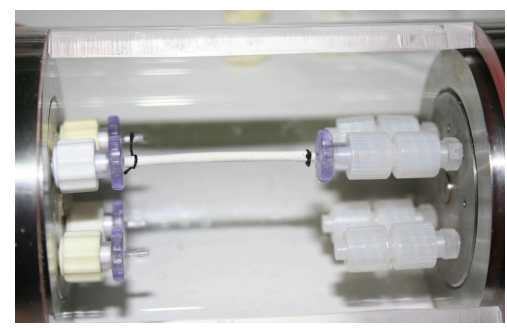

9. Position bioreactor cap so that it is centered on the window.

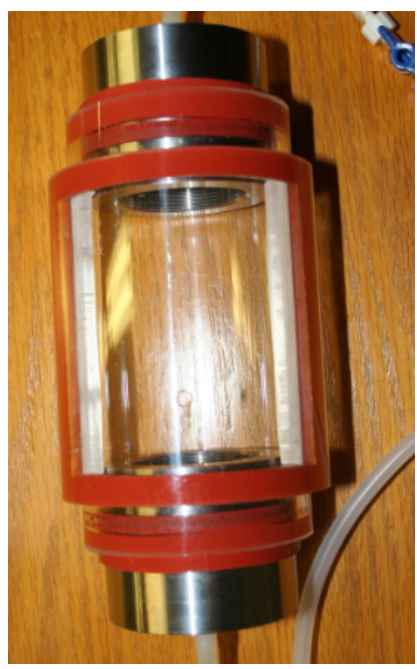


10. Apply hose clamps around both left and right edges and middle of the bioreactor cap.

11. Attach outlet barbs to each of the outer caps

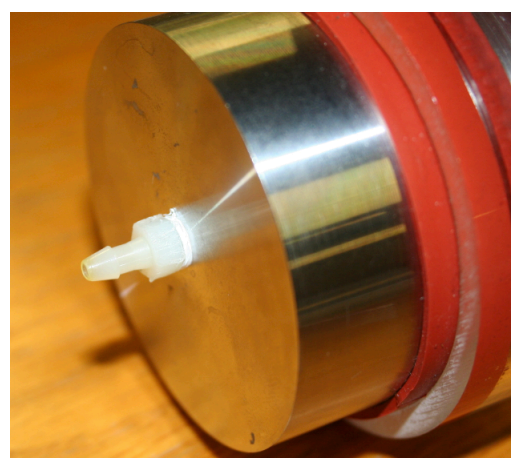

12. Tubing: Attach inlet, outlet, and pump tubing

Inlet Tubing: connect one end of the inlet tubing to the outlet connection of the pump tubing (1). Then connect the other end to the inlet of the bioreactor (2). Outlet Tubing: There are three ends in the outlet tubing. Connect one end to the outlet of the bioreactor (3), another end should be connected to one of the transmural ports on the side of the bioreactor (4). The last end should be connected to the outlet port of the media reservoir (5).

Pump Tubing: Connect the inlet connection of the pump tubing to the inlet port of the media reservoir (6). The outlet connection should be connected to the inlet tubing of the bioreactor. 


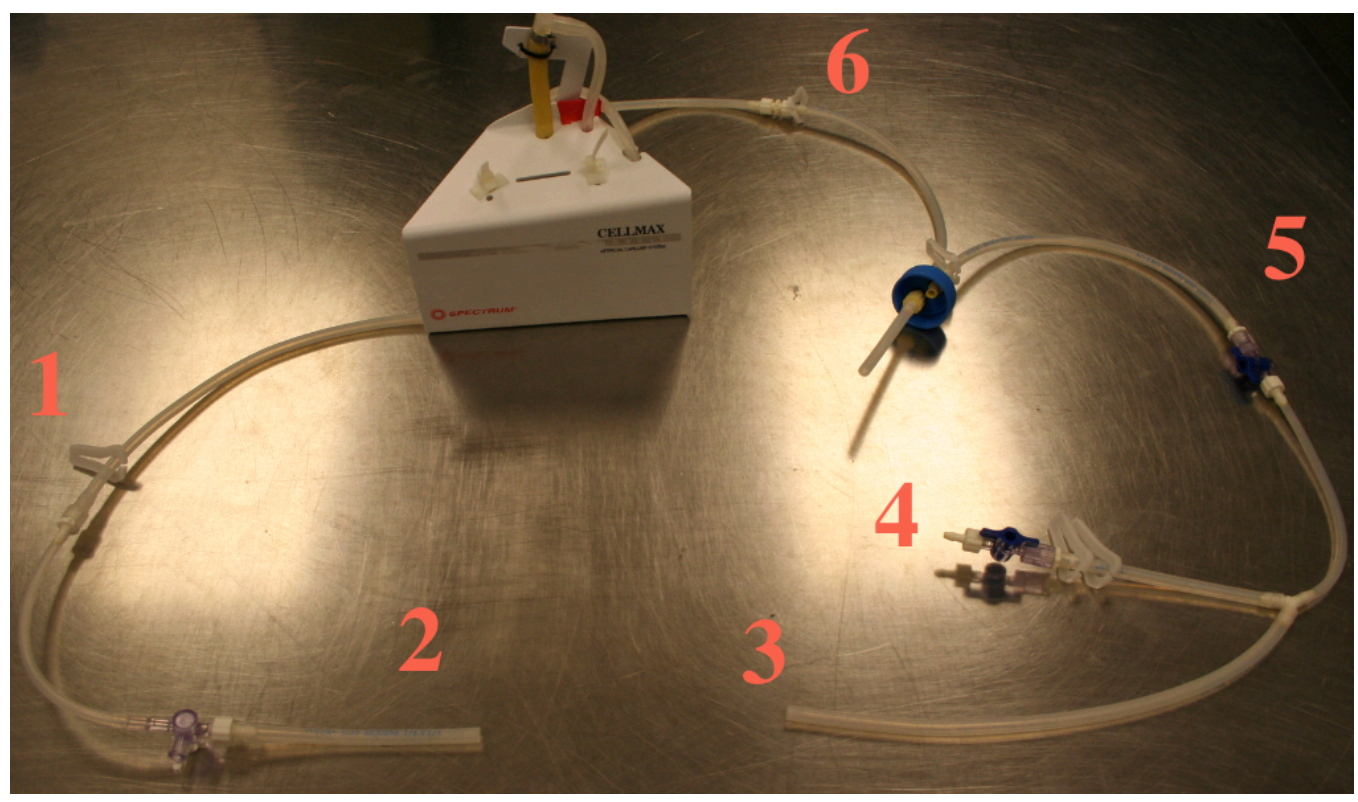

13. Glass Funnel:

Disconnect the transmural tubing at the Y-connector barb and slide the tubing onto the end of the glass funnel.

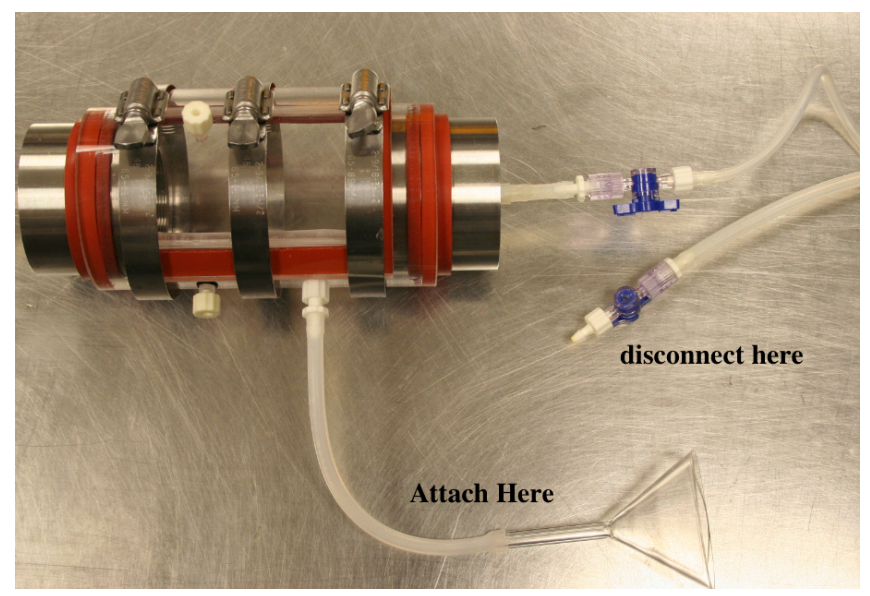

14. Be sure to leave one transmural port along with the bioreactor cap port open to allow EtO gas entry into the bioreactor (if a sterile set-up is required). If this is a nonsterile set up, close both ports with end caps.

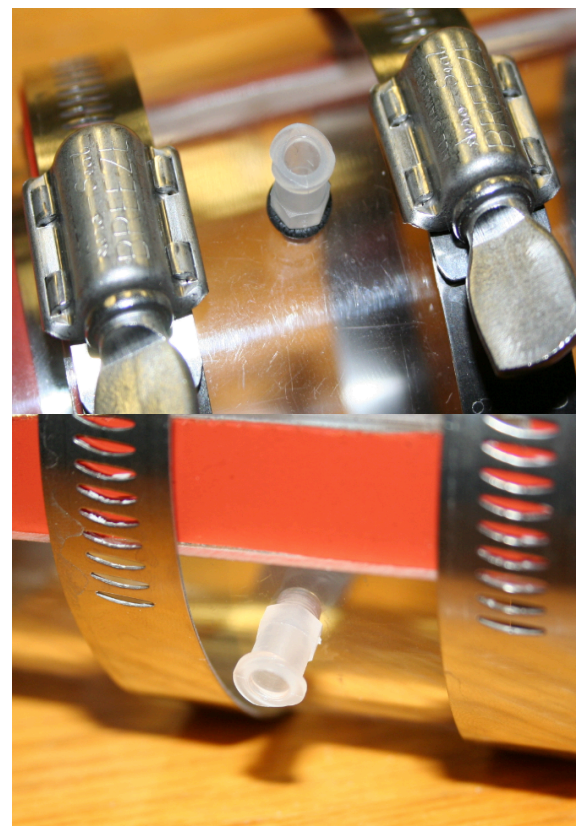




\section{Scaffold Assembly Protocol}

\begin{tabular}{|l|l|l|l|}
\hline Component & Company & Part Number & Quantity \\
\hline Qosina Barbs & Qosina & 11733 & 2 (per scaffold) \\
\hline Silk-Suture Thread & & & $\begin{array}{l}\text { Depends on \# of } \\
\text { scaffold }\end{array}$ \\
\hline PLGA Scaffold & Cal Poly & N/A & $\begin{array}{l}\text { Depends on } \\
\text { experiment } \\
\text { requirements }\end{array}$ \\
\hline
\end{tabular}

1. Cut PLGA scaffold to 1.6 " in length. Be sure not to crush scaffold lumen or deform the scaffold (beware of kinks).

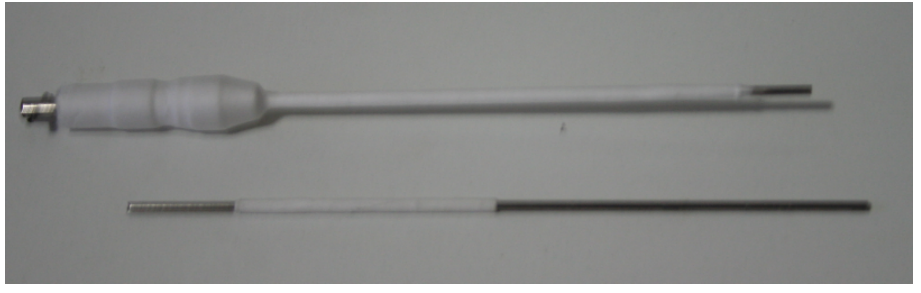

2. Slip scaffold into one Qosina barb and suture using three-knots to fully secure scaffold tightly onto barb. Repeat process to secure the other end to finish scaffold assembly.

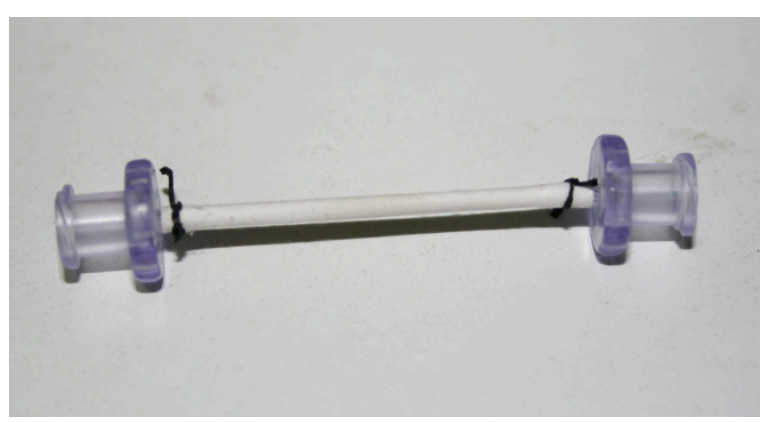

3. With the secured scaffold on the two Quosina barbs, the scaffold will now be ready to be inserted into the bioreactor during the Assembly Protocol. 


\section{Sterile Experiment Protocol}

Duration: 24 hours or 7-Days

Scaffold: PLGA

Cell Type: 3T3 Fibroblast

\section{Preparation 1-week prior to set up}

1. Determine target number of cells and passage schedules.

2. Thaw cells.

3. Package and gas

sterilize bioreactor

system.

4. Prepare bioreactor

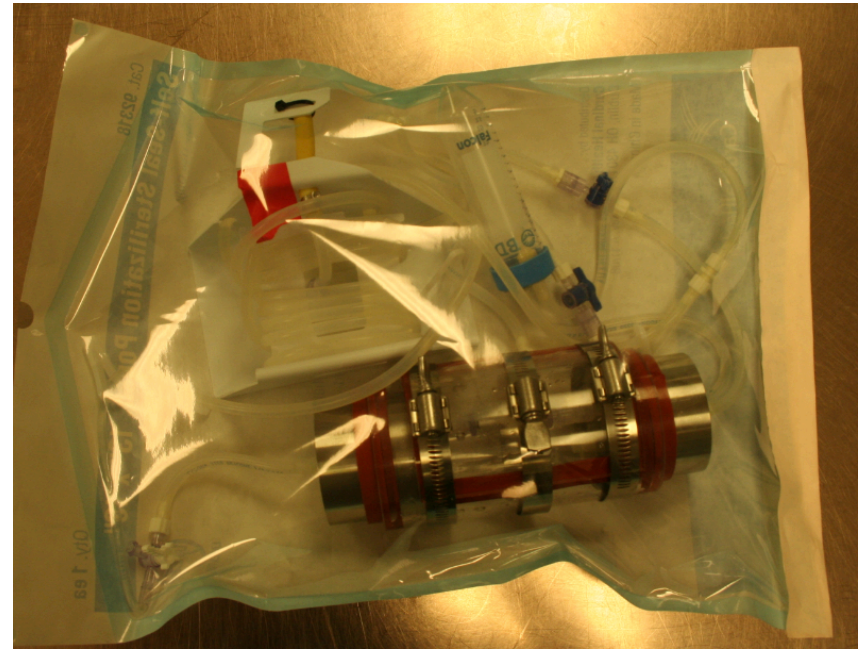
media.

5. Prepare conditioning media.

\section{Set-up day: bioreactor conditioning}

6. Place packaged bioreactor on the right side of the hood.

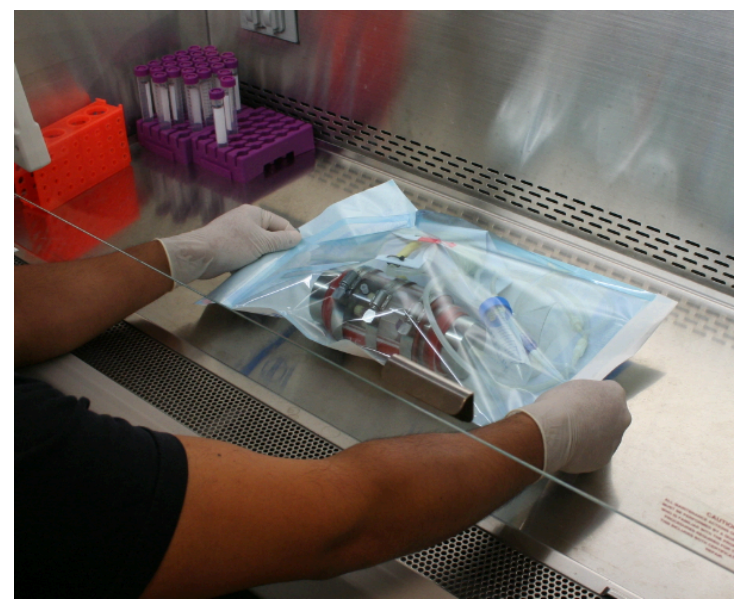


7. Peel back blue film opening the package to the right.

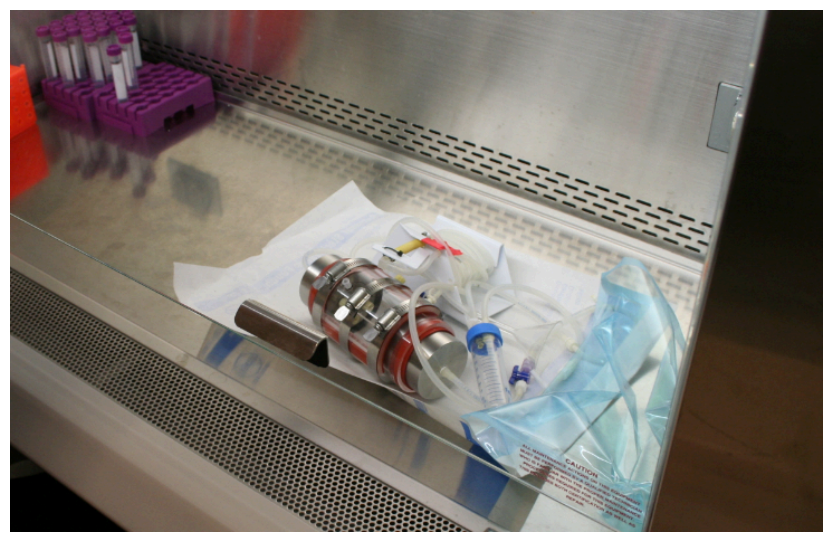

8. Locate the glass funnel used to insert bioreactor media into the bioreactor chamber.

9. Proceed to pour bioreactor media into bioreactor chamber. Be sure not to pass hands over exposed fittings ( $\mathrm{Y}$-connector barb) or ports.

10. When bioreactor media fully submerges all fittings, disconnect the glass funnel from tubing and reconnect the tubing back to the $\mathrm{Y}$ connector barb.
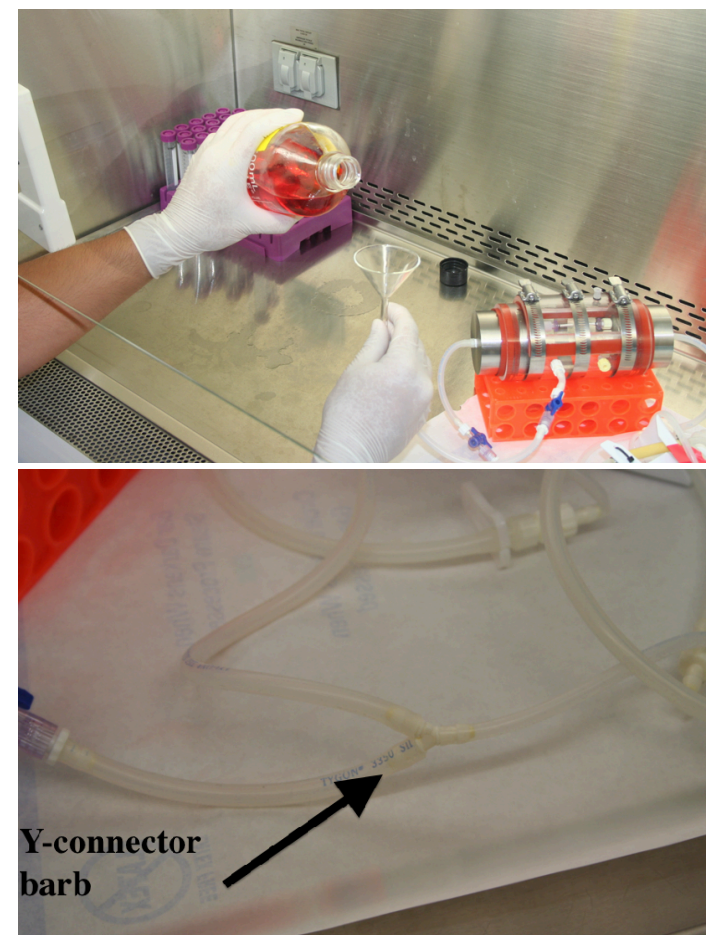

11. Fill media reservoir with $50 \mathrm{ml}$ of conditioning media. Prepare another 50 $\mathrm{ml}$ conical with conditioning media

\section{Preparing the large incubator}

12. Spray down area being used. Careful not to interfere other culture set-ups that are currently being done. 
13. Spray down pumping system and place into incubator.

14. Bring the bioreactor, pump tubing, and media reservoir into large incubator.

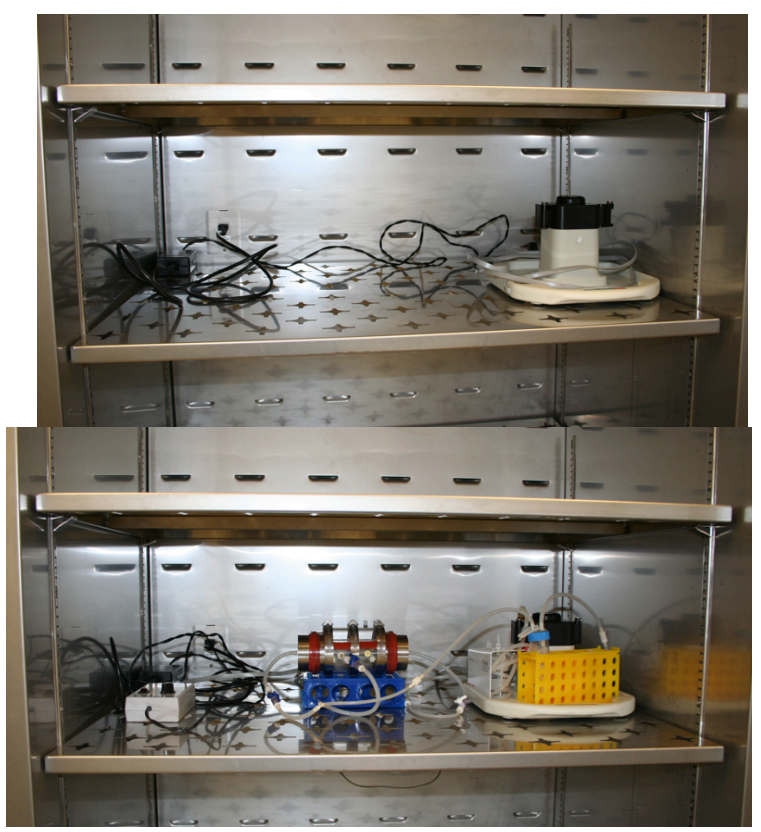

15. Turn pump to setting 1 to

prime the bioreactor letting all the air out of the system.

16. Watch media reservoir level to make sure that air is not being pumped. If media level is low during priming of the system, replace the $50 \mathrm{~mL}$ conical with more conditioning media.

17. Once primed, turn off transluminal flow by switching the one-way stopcock perpendicular to the path of flow.

18. Maintain transmural flow for 24 hours.

19. Close incubator and set $\mathrm{CO}_{2}$ levels back to $5 \%$

\section{Preparation for sodding cells}

20. Once cells have been trypsinized and deactivated with culture media transfer cells suspension into 1 trough. Prepare another trough full of culture media to chase the cell suspension. Be careful not to pass your hands over any part of the trough.

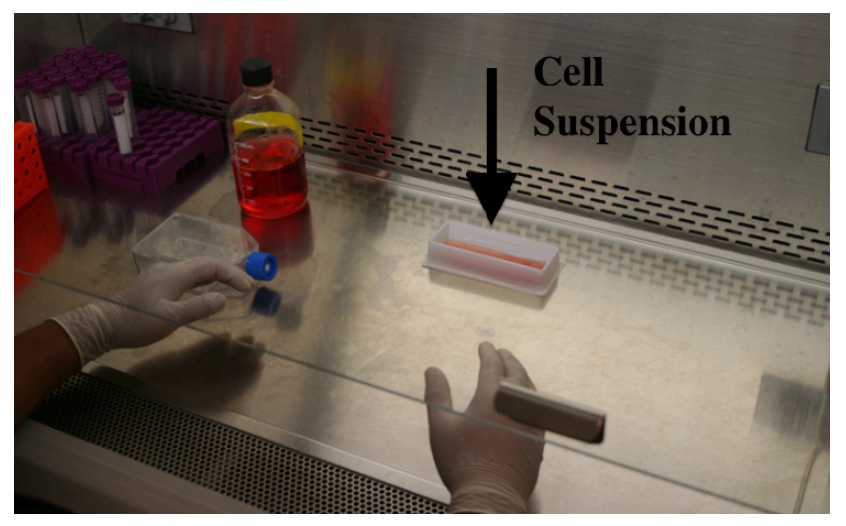

21. Use a $10 \mathrm{~mL}$ syringe to sod the cells into the bioreactor.

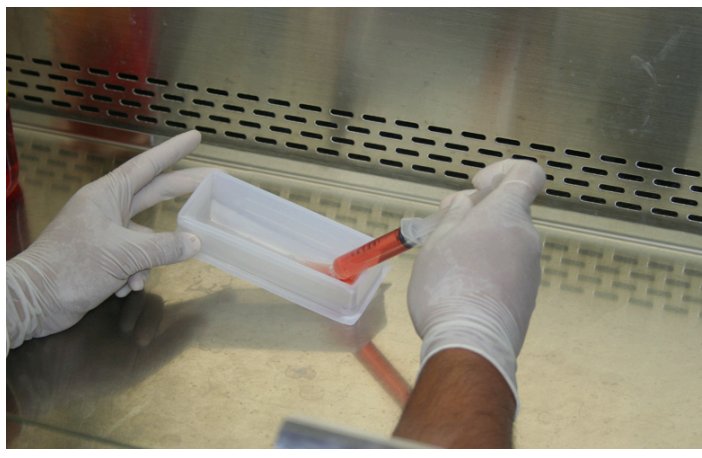


22. When both cell syringe and chase media syringe is ready. Open the incubator and stop the pump.

23. Syringe cell contents slowly into the induction port of the three-way stopcock. Be sure to stop back flow by switch the off position back to the pump tubing. Close induction port once finished with injection.

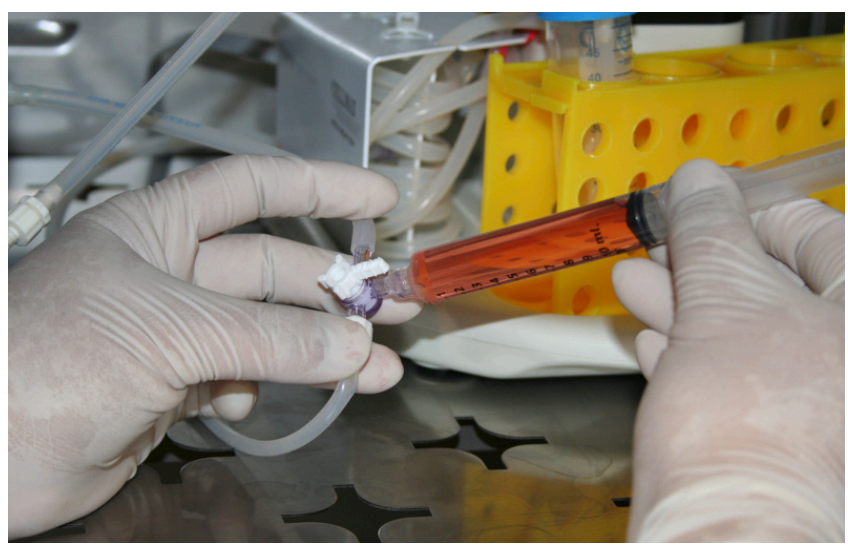

24. Syringe chase media through the same process as the previous syringe.

Close induction port once finished with injection.

25. Turn pump back on to setting 1 .

26. Allow transmural flow for 1 hour.

27. After the hour, open the incubator, turn off pump, and switch from transmural to transluminal for the duration of the experiment.

28. Maintain flow conditions at setting 1 for the duration of the experiment.

29. Close incubator and set $\mathrm{CO}_{2}$ levels back to $5 \%$

\section{For 7-Day Cultures}

30. Replace culture media every other day once cells are sodded into the system. 


\section{Bioreactor Take Down Protocol}

1. Close $\mathrm{CO}_{2}$ tank prior to opening the large incubator.

2. Using hose clamps and switchvalves located on one- and three-way stop cocks, close off flow at the inlet tubing-pump tubing interface and at the inlet connection of the pump tubing and the inlet port of the

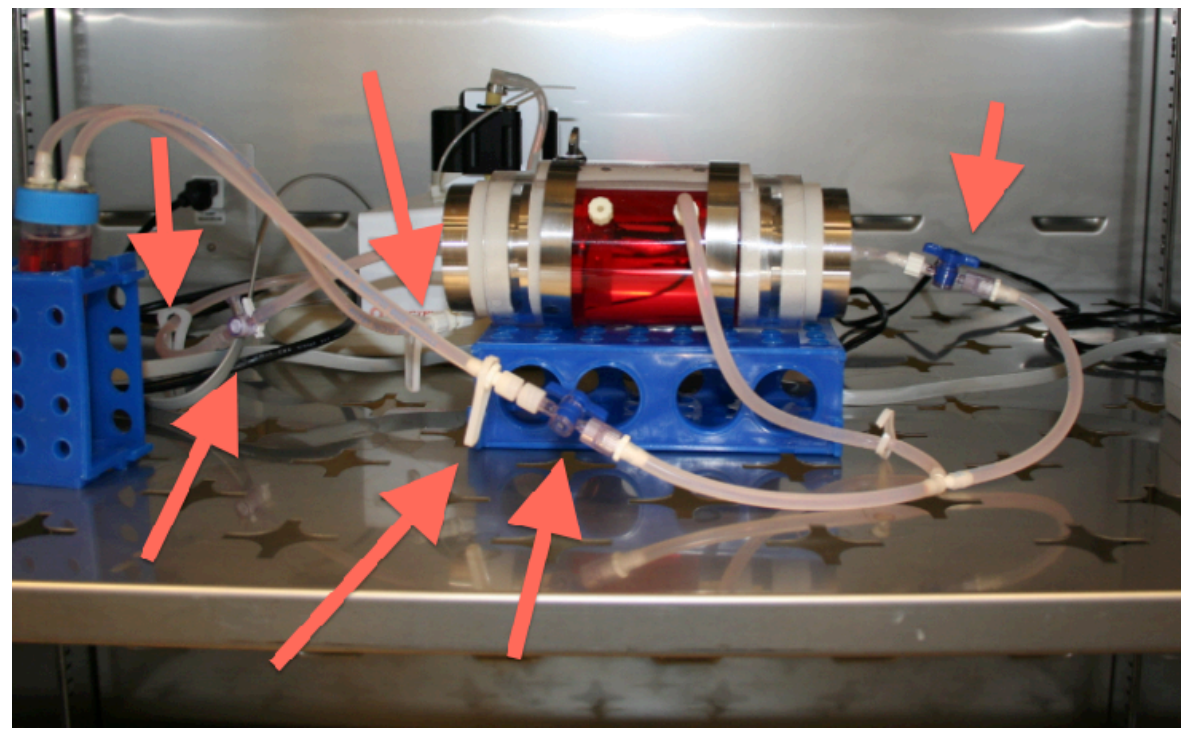
media reservoir.

This will allow detachment of the bioreactor from the pump tubing and allows the user to bring the bioreactor and media reservoir to the sink. Red arrows indicate areas that need to be clamped or one-way valves that need to be switched to stop flow.

3. Scaffold Retrieval: The user can retrieve the scaffold(s) either using a razor blade to cut the scaffold out of the bioreactor, or by draining the bioreactor fluid from the bioreactor chamber and disconnect one of the of scaffold ends from the outer cap. This will allow the user to disconnect both outer caps from the outer housing and remove the scaffolds while intact on the Quosina barbs.

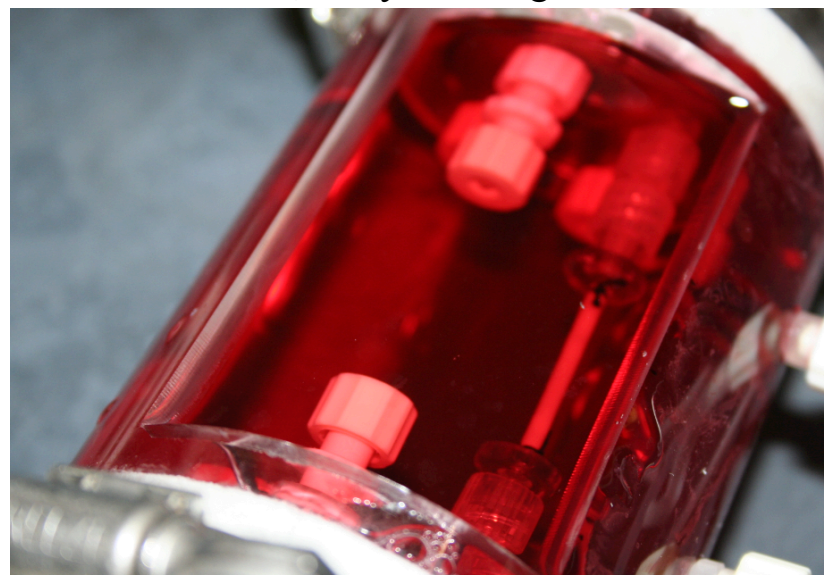

4. Once scaffolds are removed, drain all bioreactor and cell media from tubing, outer caps, and pump tubing, down the sink. Be sure to chase with bleach.

5. Rinse all components with water and place all small fittings into a small beaker with mili-Q water over night.

6. Let larger components including outer caps, scaffold caps, inlet and outlet tubing, and outer housing to dry on the lab bench over night.

7. Pump water through the pump tubing several times and then allow air to be pumped so that tubing can be dried over night. 


\section{Cell Viability Protocol Single-Scaffold Set-Up}

1. Take bioreactor out of incubator

2. Be sure $\mathrm{CO}_{2}$ tank is off

3. Place in sink and drain transmural fluid

4. Unscrew scaffold from end caps and remove scaffold from bioreactor

5. Cut scaffold

horizontally through the

diameter of the scaffold. One

half goes to $2 \mathrm{ml}$ of formalin

15 minutes. Another half

goes into $3 \mathrm{ml}$ of trypsin 6-7

minutes.

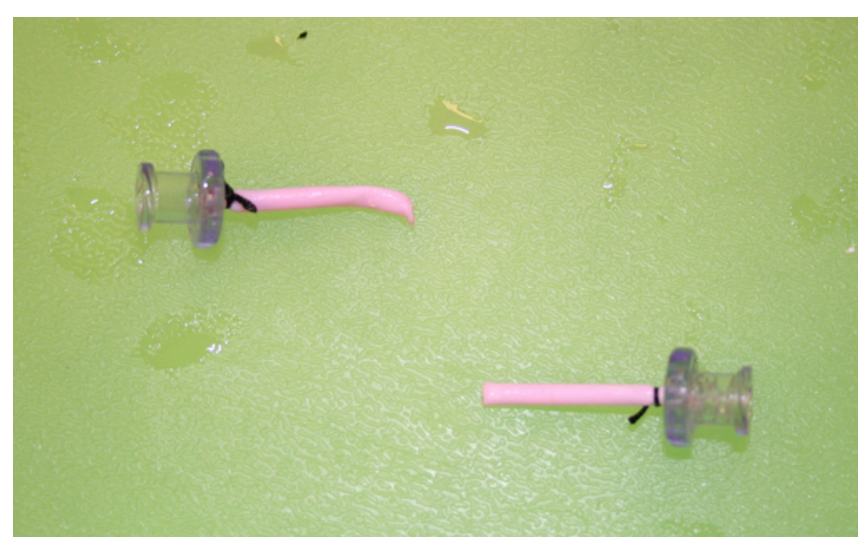

\section{Scaffold in formalin}

6. Extract scaffold from formalin and remove from barbs.

7. Cut scaffold in half longitudinally.

8. Wash in PBS twice.

9. Put in BBI Solution for 25 minutes.

Make BBI solution in a $15 \mathrm{ml}$ conical (wrapped in foil). Use small tube of stock solution and dilute 1:1000 with milli-Q water (10 $\mu \mathrm{L}$ stock solution in $10 \mathrm{~mL}$ water). Take scaffold and place it into $15 \mathrm{ml}$ conical of BBI solution for 25 minutes.

\section{Scaffold in trypsin}

10. Place scaffold in $3 \mathrm{ml}$ of trypsin for 6-7 minutes. Agitate and flush lumen using pipette.

11. Deactivate with $3 \mathrm{ml}$ of fibroblast media.

12. Flush lumen to get all cells out.

13. Remove scaffold and dispose in biohazard bin.

14. Spin down at $1000 \mathrm{rpm}$ for 5 minutes.

15. Aspirate the supernatant and re-suspend the cell pellet in $0.5 \mathrm{~mL}$ of fibroblast media.

16. Place $150 \mu \mathrm{l}$ cell solution $50 \mu \mathrm{l}$ of trypan blue.

17. Place $20 \mu 1$ into cellometer slide and view.

\section{Imaging}


18. BBI: Turret position 4, FW1 position 1, FW2 position 1.

19. Trypan blue: follow cellometer directions in 209a. 


\section{Cell Viability Protocol Multi-Scaffold Set-Up}

Scaffolds: 4

1. Take bioreactor out of incubator

2. Be sure $\mathrm{CO}_{2}$ tank is off

3. Place in sink and drain transmural fluid

4. Unscrew scaffold from end caps and remove scaffold from bioreactor

5. Transfer as many intact scaffolds in formalin or trypsin.

6. Scaffold should be in a $15 \mathrm{~mL}$ conical of trypsin for 6-7 minutes.

7. Scaffold should be in a $15 \mathrm{~mL}$ conical of formalin for 15 minutes.

\section{Scaffold in formalin}

8. Extract scaffold from formalin and remove from barbs.

9. Cut scaffold in half longitudinally.

10. Wash in PBS twice.

11. Place in BBI solution.

Make BBI solution in a $15 \mathrm{ml}$ conical (wrapped in foil). Use small tube of stock solution and dilute 1:1000 with milli-Q water (10 $\mu \mathrm{L}$ stock solution in $10 \mathrm{~mL}$ water). Mix by inverting.

12. Take scaffold and place it into $15 \mathrm{ml}$ conical of BBI solution for 25 minutes

\section{Scaffold in trypsin}

13. Place scaffold in $6 \mathrm{ml}$ of trypsin for 6-7 minutes. Agitate and flush lumen using pipette.

14. Deactivate with $6 \mathrm{ml}$ of fibroblast media

15. Flush lumen to get all cells out

16. Remove scaffold and dispose

17. Spin down at $1000 \mathrm{rpm}$ for $5 \mathrm{~min}$

18. Aspirate the supernatant and re-suspend the cell pellet in $0.5 \mathrm{~mL}$ of fibroblast media

19. Place $150 \mu 1$ cell solution $50 \mu 1$ of trypan blue

20. Place $20 \mu \mathrm{l}$ into cellometer slide and view

\section{Imaging}

21. BBI: Turret position 4, FW1 position 1, FW2 position 1

22. Trypan blue: follow cellometer directions in 209a. 


\section{Cell Viability Protocol Invitrogen Assay}

\section{Prior to bioreactor take down}

1. Prepare a Live/Dead stain solution with a Calcein AM concentration of $1.0 \mathrm{uM}$ and an EthD-1 concentration of $2.0 \mathrm{uM}$ in DCFPBS (approximately $7 \mathrm{ml}$ of staining solution will be needed for each scaffold).

a. The following dilution will result in a $1.0 \mathrm{uM}$ Calcein AM and 2.0 uM EthD-1 solution with a total volume of $20 \mathrm{ml}$ when using the Live/Dead Viability/Cytotoxicity Kit (Invitrogen, L-3224).

i. Stock solution: $20 \mathrm{~mL}$ of DCF-PBS, $20 \mu 1$ of EthD-1, $5 \mu \mathrm{L}$ Calcein AM.

2. Transfer $7 \mathrm{~mL}$ of the staining solution into a $15 \mathrm{~mL}$ foil wrapped conical tube for each scaffold being assessed. Store conical in fridge before use.

\section{During bioreactor take down}

3. Transfer PLGA scaffold into $7 \mathrm{~mL}$ of trypsin for 6-7 minutes. Be sure to agitate the conical several times to remove cells from the inner lumen of the scaffold.

4. After sitting in trypsin, deactivate with $7 \mathrm{~mL}$ of culture media. Be sure to flush lumen with culture media to fully remove cells from inner lumen.

5. Dispose of the scaffold in the biohazard bin and spin down $14 \mathrm{~mL}$ cell suspension at 1000 RPM for 5 minutes.

6. Aspirate the supernatant and resuspend the cell pellet in $5 \mathrm{~mL}$ of the Live/Dead staining solution.

7. Let the scaffold sit in the staining solution for 30 minutes at room temperature.

8. After 30 minutes transfer two $10 \mu \mathrm{L}$ samples of the suspension to Grids A and B in a disposable hemocytometer.

9. Image both grids at $40 \mathrm{X}$ magnification using the fluorescent microscope in 192-106B.

a. Live cell images: use FW1 position 2, FW2 position 2.

b. Dead cell images: use FW1 position 2, FW2 position 3.

c. Use brightfield to capture hemocytometer grid. 


\section{Cell Viability Rhodamine Protocol}

1. Take bioreactor out of incubator

2. Be sure $\mathrm{CO}_{2}$ tank is off

3. Place in sink and drain transmural fluid

4. Unscrew scaffold from end caps and remove scaffold from bioreactor

5. Transfer scaffold into $7 \mathrm{~mL}$ of formalin for 15 minutes.

6. Extract scaffold from formalin and remove from barbs.

7. Cut scaffold in half longitudinally.

8. Wash in PBS twice.

9. Permealize in .1\% tritonX-100 (.2 ml of triton in 1.8 in PBS) (in the fridge in the "PECAM" Tupperware) for 5 minutes.

10. Wash in PBS twice

11. Stain with Rhodamine (in the freezer, wrapped in foil) for 20 minutes. For dilution 20ul of stock into $800 \mu \mathrm{l}$ of PBS.

12. Wash with PBS. 


\section{Appendix E - Small-Scale Barbs}

To further create a robust model of the BBB, introducing more

physiological components is important to further validate the system. To progress

further toward that notion, two students, Matt Rogers and Nathan Ferrier, developed small-scale barb fittings that allowed the use of $1 \mathrm{~mm}$ inner diameter scaffolds. Previously, the bioreactor system utilized $2 \mathrm{~mm}$ inner diameter Qosina barb fittings that were donated for this project. With the creation of these smaller scale barb fittings, cellular interaction as well as further development of a BBB can be achieved. The fittings were made to improve upon the previous Qosina barb fittings used to secure the scaffolds. The design requirements were to have a fitting that would be compatible with sterilization techniques used in the lab, ability to produce flow, and allow the use of smaller diameter scaffolds. The material chosen was 316-stainless-steel. This allowed compatibility to both sterilization techniques including EtO and autoclave. The fitting was designed to be a hex shape to provide sufficient installation into the scaffold cap. The fitting was able to allow flow through its inner lumen. A barb was machined at the end to allow sutures to wrap around the fitting to secure scaffolds. Cellular testing conducted with the small-scale barb fittings were not included in this thesis, however these tests can provide experiments for future testing in the laboratory. Cellular testing can evaluate cell distribution with the use of these barb fittings, and further assess if cell distribution is better compared to the protocols under the use of the Qosina barbs. If the small-scale barbs do not show improved results, changes to protocols must be made to achieve better distribution of cells. 